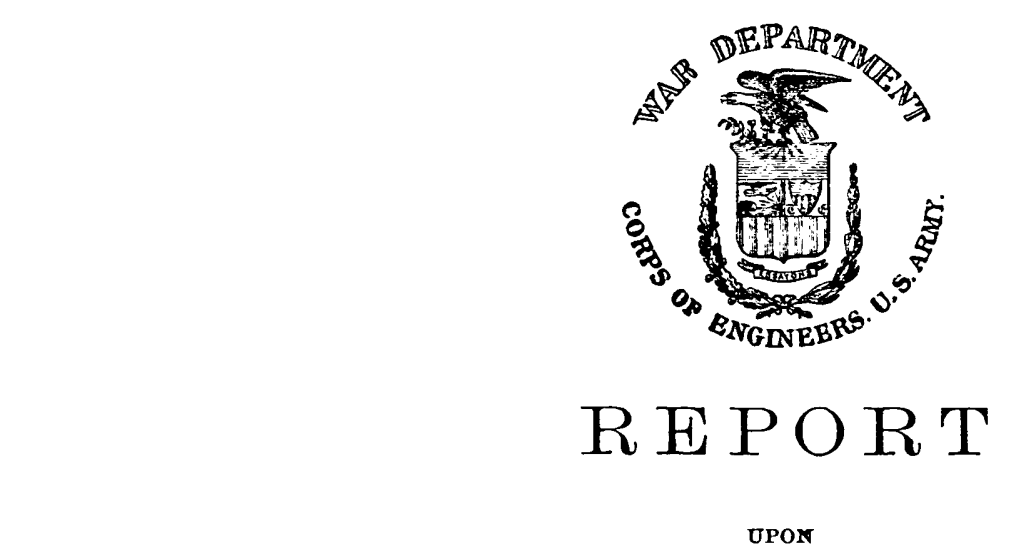

\title{
THE DE'TERMINATION
}

OF THE

\section{ASTRONOMICAL CO-ORDINATES}

OF

THE PRINIARY STATIONS AT CHEYENNE, WYOMING TERRITORY, AND COLORADO SPRINGS, COLORADO TERRITORY,

\author{
MADE DURING \\ The Years 1872 and 1873, \\ GEOGRAPHICAL AND GEOLOGICAL EXPLORATIONS AND SURVEYS WEST OF THE \\ ONE HUNDREDTH MERIDIAN.
}

FIRST LIEUTENANT GEORGE M. WHEELER, CORPS OF ENGINEERS, IN CHARGE.

Dr. F. KAMPF and J. H. CLARK, CIVILIAN astroNomical assistants.

W A S H I N G T ON:

GOVERNMENT PRINTING OFFICE.

1874 . 
. 


\section{TABLE OF CONTENTS.}

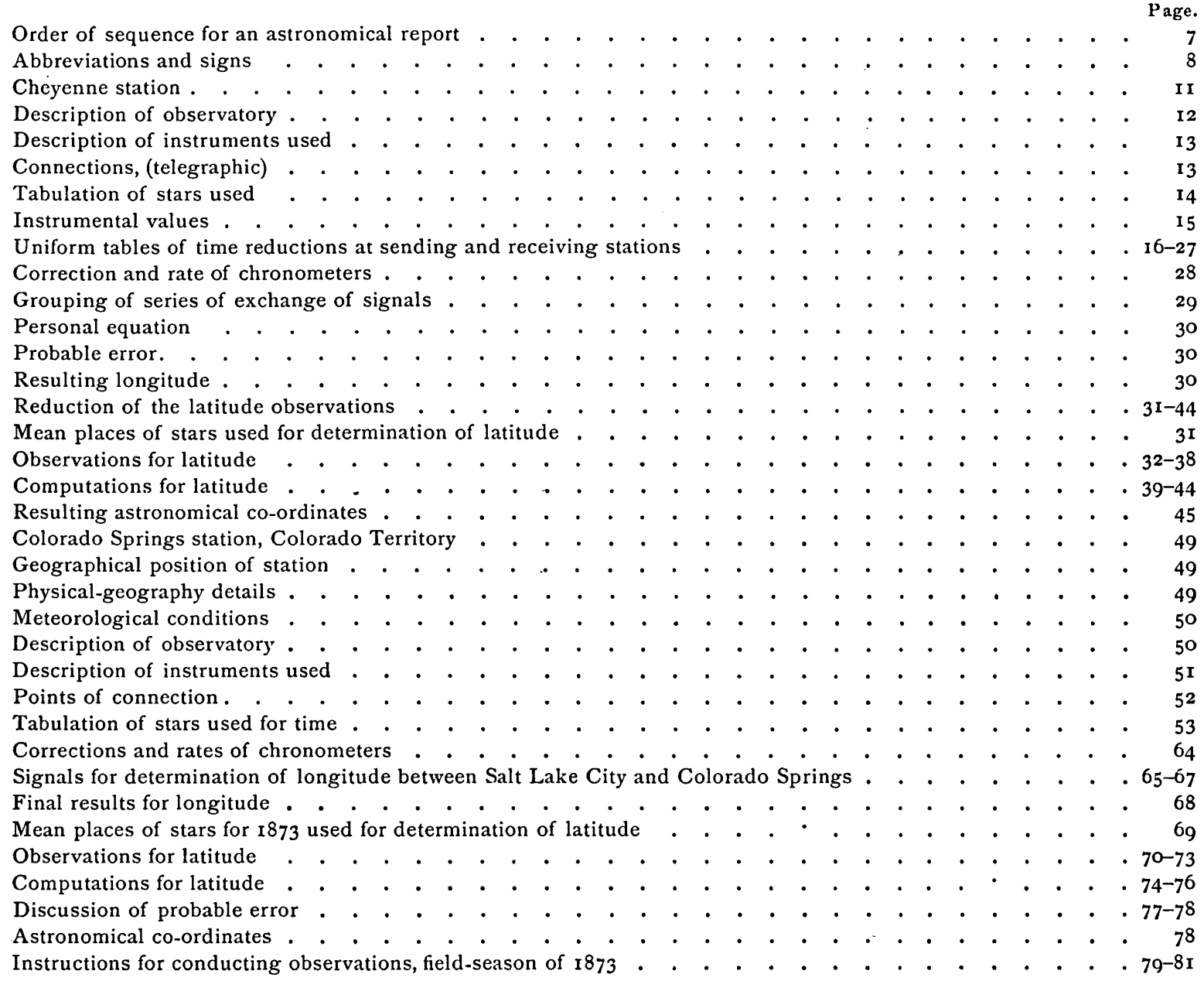




.




\section{Office of the Chiff of Engineers, Washington, D. C., February i 3 , I 874 .}

SIR: Lieut. George M. Wheeler, Corps of Engineers, has sent to this office a report embodying the results from the astronomical observations made at Cheyenne, Wyoming Territory, and Colorado Springs, Colorado Territory.

As this report contains information of value to officers engaged upon explorations, surveys, and reconnaissances in the western country, I have respectfully to recommend that it be printed at the Government Printing-Office, and that five hundred copies be furnished on requisition from this office.

Very respectfully, your obedient servant,

$$
\begin{gathered}
\text { A. A. HUMPHREYS, } \\
\text { Brigadier-General and Chief of Engineers. }
\end{gathered}
$$

Hon. W. W. Belknap, Secretary of War.

Approved by the Secretary of War, February 14, I874.

H. T. Crosbr, Chief Clerk.

\section{United States Engineer Office, Geographical and Geological Exploratrions and Surveys \\ West of the IOOTH Meridian, Washington, D. C., February 9, I874.}

SIR: I have the honor to forward herewith a report embodying the results from the astronomical observations made at Cheyenne, Wyoming Territory, and Colorado Springs, Colorado Territory. They are typical stations for the years 1872 and 1873 , although not selected because of probable errors that are a minimum.

Attention is invited to the methods employed and the order of sequence in reporting the results.

Uniformity of plan seems to be a matter of so great importance in the prosecution of astronomical work in the western interior that the one now in use is submitted for consideration as a step at least in this direction.

Minor features of the methods are still to be perfected; yet the errors of starplaces, from observation, and those known as instrumental, have now become reduced to such small and nearly equable values, that it seems desirable that some general and uniform plan should be adopted for the report at least, if not for the manner of conducting the observations.

The present one is submitted with extreme diffidence, and will probably demand certain changes in the light of future experience.

At Colorado Springs a heavy stone monument and observing-pier, with meridianmarks, have been established. 
The same has been done at the greater number of the other points occupied as main field-stations in the years 1871,1872 , and 1873 .

These meridian-lines may serve various purposes in checking future surveys in adjacent areas.

Very respectfully, your obedient servant,

GEO. M. WHEELER,

Brig. Gen. A. A. Humphreys, Lieutenant of Engineers, in Charge.

Chief of Engineers, United States Army.

Note.-The results from the observations made during the field-seasons of $187 \mathrm{I}$, 1872 , and 1873 , at the remaining primary stations, twenty-two in number, will appear in Volume II of the Reports of the Survey, as proposed in the Annual Report of the Chief of Engineers for I873, Appendix EE. 
ORDER OF SEQUENCE

FOR AN

\section{A S T R O N O M I C A L R E P O R T}

AT

MAIN OR PRIMARY FIELD-STATIONS.

Longitude by Telegraph; Latitude by Zenith Telescope, (Talcott's method.)

I. Geographical position of station.

2. Physical-geography details; especially all physical peculiarities.

3. Meteorological conditions, both general and special; the latter while observations were made.

4. Description of observatory; including personnel of party, name of telegraphoperator, and name of telegraph-company whose wire has been employed.

5. Description of instruments used.

6. Points with which connections were made; nights of observation, and observers; also name of computer or computers.

7. Tabulation of stars used, and number of observations.

8. Instrumental values; circumstances of telegraphic communication, $i$. e., length of circuit, number of batteries, repeaters, \&c.

9. Uniform tables of time-reductions at receiving-station.

Io. Uniform tables of time-reductions at sending-station.

I I. Grouping of series of exchange-signals, including means of single and serial results.

I 2. Personal equation.

I 3. Probable error by least squares.

I 4. Resulting longitude.

I 5. Reduction of the latitude-observations properly grouped, with discussion of results.

16. Resulting astronomical co-ordinates. 


\section{ABBREVIATIONS AND SIGNS.}

$a, b, c,=$ azimuth, level, and collimation corrections.

$\mathrm{A}, \mathrm{B}, \mathrm{C}$, = azimuth, level, and collimation factors.

$\mathrm{T}=$ observed time reduced to the mean of wires and corrected for rate.

$\mathrm{T}^{\prime}=$ observed time corrected for instrumental errors.

$\mathrm{AR} .=$ apparent right ascension of star.

$\Delta_{0} \mathrm{~T}=$ resulting error of the chronometer after the mean of the wires is corrected for rate and level.

$\Delta \mathrm{T}_{0}=$ adopted mean error of chronometer.

$\delta \mathrm{T}=\Delta \mathrm{T}_{0}-\Delta_{0} \mathrm{~T}$.

$\Delta \mathrm{T}=$ error of the chronometer.

$v=$ difference between mean final correction of chronometer and $\Delta \mathrm{T}$. 


\section{R E POR T}

on

\section{ASTRONOMICAL OPERATIONS,}

CONDUCTED DURING

THE FIELD-SEASON OF 1872,

AT THE

MAIN OR PRIMARY FIELD-STATION, CHEYENNE, WYOMING TERRITORY,

AND

\section{DEDUCTION OF RESULTS.}

BY

DR. F. KAMPF AND J. H. CLARK, 



\section{United States Engineer Office, Geographical and Geological Explorationg and Surveys West of the iooth Meridian, Washington, D. C., January I, I 874 .}

\section{CHEYENNE, WYOMING TERRITORY.}

\section{(i.) Geographical Position of Station.}

The station at Cheyenne, the latitude of which is N. $41^{\circ} 7^{\prime} 46^{\prime \prime} .62$, and its longitude $28^{\mathrm{m}} 19^{\mathrm{s}} .44$ east of the Mormon observatory at Salt Lake, is situated in the western part of the town, in lot I I, block 4I3, and is northwest from the junction of the branchroad leading to the depot of Fort D. A. Russell with the main stem of the Union Pacific Railroad.

The town of Cheyenne, on the north side of the railroad, is the capital of Wyoming Territory; and besides the Union Pacific Railroad with its branch to Fort Russell, has a railroad-connection with Denver, Colorado. These facilities make it a flourishing place, and it possesses already, in addition to its public buildings, quite a number of substantial business-houses and attractive private residences.

A fine hotel, engine-houses, workshops, and other similar improvements, are among the sources of prosperity which spring directly from the railroad-company.

Besides the traffic with Fort Russell, it is the depot for Red Cloud's agency, Fort Laramie, and the settlements on the North Platte.

The surrounding region affords unlimited pasturage, and cattle are said not only to subsist but grow fat on it during the whole year.

Agriculturally there is but little promise outside of what may be accomplished by irrigation.

\section{(2.) Physical-Geography Details.}

The site of Cheyenne is nearly or quite level; northward, however, there is a gradual swell of the land, and within a few miles it cuts off the extensive view one is accustomed to on the great plains. Eastward and southward there is the usual rolling and slightly-broken prairie; but in the southwest, Long's Peak, already white this September with snow, some seventy miles distant, looms up boldly above the horizon. West and northwest, low mountains, many of which mark the rim of the Laramie Plains, are just visible. Crow Creek, a moderate stream coming down from the foothills of this rim, forms a valley immediately west and south of the town, working its way apparently through mountain-drift, as the direct eruptive force of the Rocky Mountain system seems not to have reached so far east in this latitude.

\section{(3.) Meteonological Conditions.}

The determination of this station occupied the first three weeks in the month of October, I872. There were no rains, nor any clouds, and, with the exception of two or three windy nights, the elements presented no obstacle whatever to a continued series of observations. In the early morning it would become cold enough to form 
ice, making it sufficiently uncomfortable to restrain the ardor of an observer from extending his observations into the small hours of the night. From the experience of this season, and that of many others which I have undergone in former years in astronomical work, both on the plains and in the mountains, I am of the opinion that the best time of the year (so far as the weather is concerned) for astronomical observations in the Territories and the far Western States occurs in September and October. They are the months that fall between the rainy season of the summer and the stormy weather of the winter.

The following table shows the direction of the wind, and the estimated force, from 7 a. m. to 7 p. m. and from 7 p. m. to 7 a. $\mathrm{m}$. The weather was clear all the time. Such an atmosphere, at an elevation of 6,000 feet, could not be otherwise than favorable for astronomical work.

\begin{tabular}{|c|c|c|c|c|c|}
\hline \multirow{2}{*}{ Date. } & \multicolumn{2}{|c|}{ General direction of wind. } & \multicolumn{2}{|c|}{ Estimated force of wind. } & \multirow{2}{*}{ Remarks. } \\
\hline & 7 a. m. to 7 p. m. & 7 p. m. to 7 a. m. & 7 a. m. to 7 p. m. & 7 p. m. to 7 a. m. & \\
\hline 1872 & & & & & \\
\hline October 5 & NW. & N. & I & $\mathbf{I}$ & \\
\hline 6 & SW. & o & I & o & \\
\hline 7 & NW. & o & $\mathbf{I}$ & o & \\
\hline 8 & NE. & o & I & o & \\
\hline 9 & SE. & o & $\mathbf{I}$ & o & \\
\hline Io & SE. & o & $\mathbf{I}$ & o & \\
\hline II & N. & 0 & I & o & \\
\hline I 2 & N. & o & I & o & \\
\hline I3 & o & o & o & o & \\
\hline $\mathrm{I}_{4}$ & NW. & NW。 & $\mathbf{I}$ & $i$ & Aurora borealis in the north \\
\hline 15 & NW. & o & 2 & o & at $\mathbf{1} \mathrm{t} .30 \mathrm{p} . \mathrm{m}$. \\
\hline 16 & NW. & o & $\mathbf{I}$ & o & \\
\hline 17 & sw. & o & $\mathbf{I}$ & o & Aurora borealis in the north \\
\hline 18 & o & o & o & o & at 9 p. m. \\
\hline I9 & W。 & o & $\mathbf{I}$ & o & \\
\hline 20 & W. & N. & 1 & 2 & \\
\hline
\end{tabular}

(4.) Description of Observatory.

A large wall-tent drawn over a framework formed the observatory. The opening for the menidien-line was furniched with a flap and curtains; the former served to protect the instrument from the weather when not in use, and the latter to keep off the wind and dust while observing. Here, and at other stations previously occupied, the opening for the meridian-line in the observatory was entirely unobstructed. Subsequently it was found to be an improvement to retain the ridge-pole (which commonly interferes but little with the completeness of the observations) as a support against the violent winds so prevalent in the comtry in which our operations are conducted. The tent was fumished with all the appliances of a field and temporary observatory, such as stools, stands, tables, and the like, and nothing was wanting as to equipment for first-

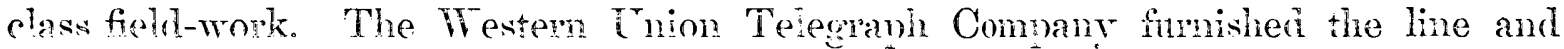
other ficilities for this station. Mr. Bates, one of the operators emplored at the

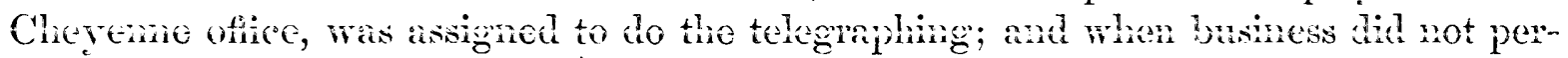


mit him to leave, which sometimes happened, Mr. Henderson, who was off duty at this hour, was obliging enough to supply his place gratuitously. Mr. F. R. Simonton was my assistant here, and kept, in conjunction with C. Herbert, an hourly meteorological record, besides his other duties as assistant.

\section{(5.) Description of Instruments Used.}

(a.) Transit.

The transit used was the meridian-zenith-instrument No. 28, made by Würdemann, a description of which is given in Dr. Kampf's report, and it was mounted on a large block of wood. The instrumental values are, for one division of the micrometerscrew, $\mathrm{O}^{\prime \prime} .6216$; of the striding-level $\mathrm{A}, \mathrm{I}^{\prime \prime} .2 \mathrm{r}$; and of the zenith-level, $\mathrm{I}^{\prime \prime} . \mathrm{IO}$

\section{(b.) Chronograph.}

The record of the observations for time, as well as the exchange of signals, with one exception (the night of the 14 th), was made by means of the chronograph, of the form contrived by Professor Harkness, United States Naval Observatory. It consists of clock-work driven by a weight, and can be adjusted to run some two hours. The regulation of the movement is effected by a steel spring, with movable balances, striking on a fly-wheel. A cylinder is attached, covered with paper, and is made to revolve once a mirute. Along this cylinder a screw carries a pen, which, being in the same circuit with the chronometer, records its breaks. The chronometric breaks, are made every second, except the sixtieth, which is omitted, to mark the minute. Removing the paper from the cylinder, both the minutes and the seconds will be found, if the instrument is working properly, recorded in parallel lines, and the culminations of the stars observed, distinctly marked by arbitrary breaks, and easily read off.

\section{(c.) Chronometer.}

'The chronometer in use here was the Negus break-circuit No. 1499. It had a gaining-rate, +o.054, hourly average, at a mean temperature of $50^{\circ}$. The breakarrangement got out of order once, but it was readily repaired, and ran the rest of the season without giving any trouble.

\section{(d.) Battery.}

A local circuit of sufficient force was produced by two cups of zinc and copper, a form known, I believe, among electricians as the Hill battery. It is simple, works a week or more without renewal, and the only possible objection to it for the purpose to which we apply it is that the sulphate is a little slow to act; particularly in cold weather.

\section{(6.) Connections.}

The observatory was west of the telegraph-office, and the main connection was effected by a loop into one of the main overland wires and put in communication with Salt Lake by a switch at Ogden. The local connections, including the chronometer, the chronograph, and the observing-keys, were made by means of a switch-board, which also receiver the main circuit. The various wires being put in their proper 
posts on this board, and the circuit closer, it required only the simple novement of sliding a switch-button to cut off or put on either circuit, and thus send the chronometric break, or receive that of the connected station on the chronographic sheet, as desixed. The nights of the 1 st, $3 \mathrm{~d}, 8 \mathrm{th}$, 9 th, roth, IIth, and 12 th were occupied on latitude, in which time 257 results were obtained. The 5th, I 4th, I6th, I 8th, I $9^{\text {th }}$, and 2 ist were successively put in on longitude. There were several other evenings when efforts were made in this direction, but failed from causes beyond my control. Mr. Austin did the observing at Salt Lake, while the observations at Cheyenne were made by myself, and also the longitude reductions for both stations. Prof. W. A. Rogers, of Harvard College Observatory, computed the latitudo-observations, and they were subsequently revised, partially recomputed, and formulated by Dr. Kampf。

\section{(7.) Tabulation of Stars Used.}

Tabulation of Stars used for Determination of Time at Cheyenne, Wyoming Territory, and Salt Lake City, Utah Tevitory.

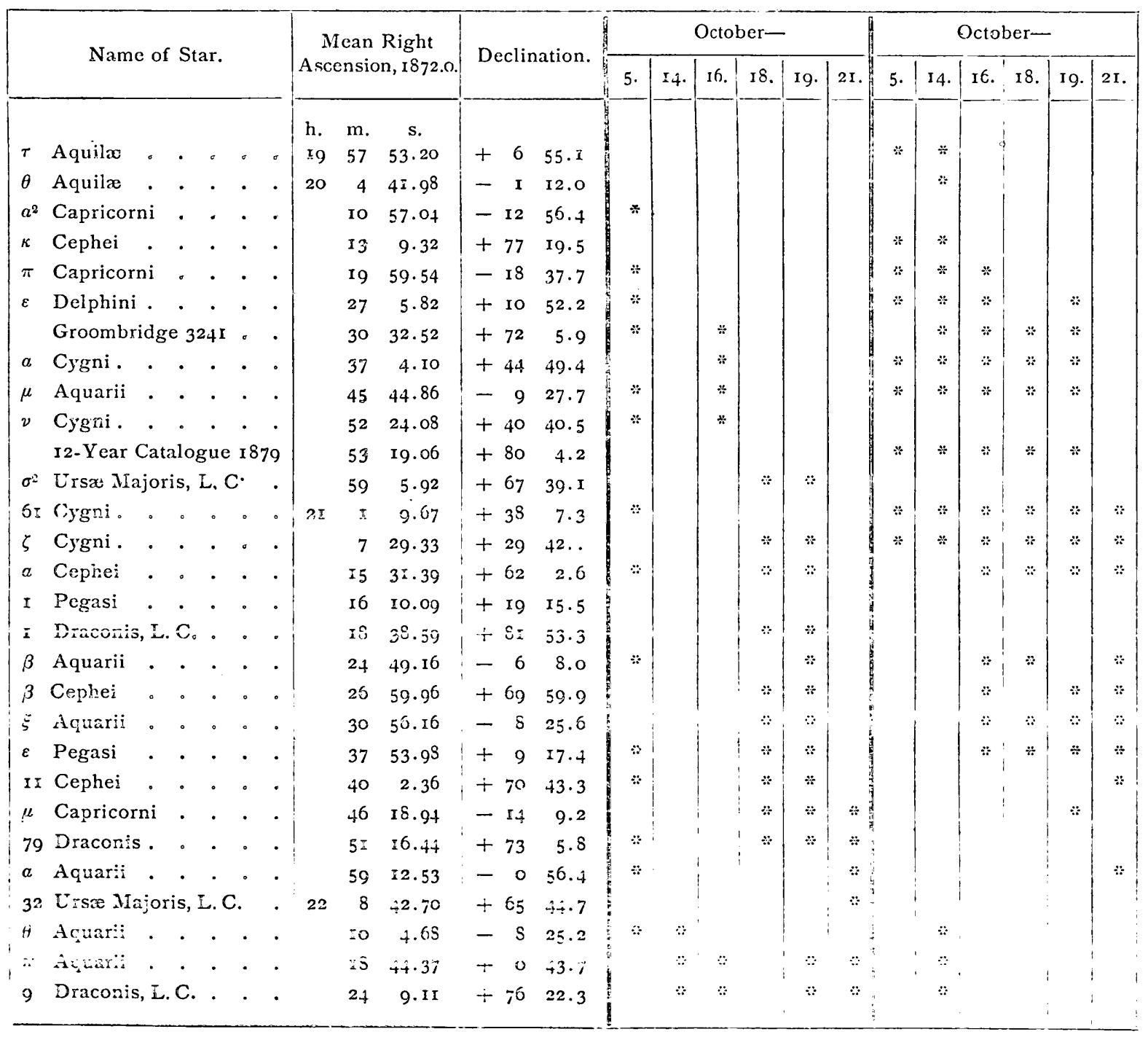


Tabulation of Stars used for Determination of Time, dec.-Continued.

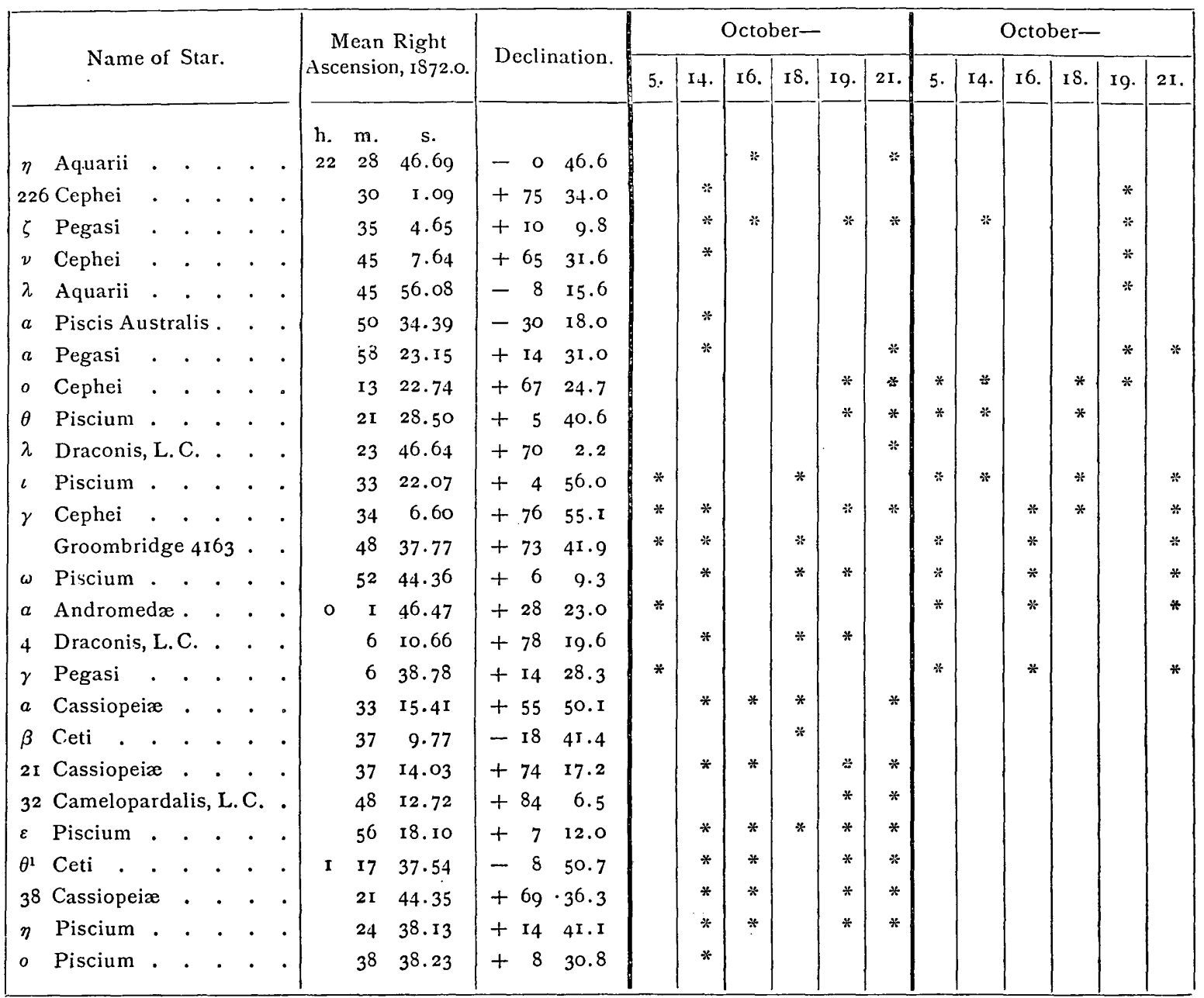

\section{(8.) Instrumental Values.}

The values of the implements pertaining to zenith-instrument No. 28 are, for one division of the micrometer-screw, $\mathrm{O}^{\prime \prime} .622$; of the striding-level $\mathrm{A}, \mathrm{I}^{\prime \prime} .2 \mathrm{I}$; and of the zenith-level, I". ${ }^{\prime \prime}$. The wire which made the circuit between the stations Salt Lake and Cheyenne, counting the distance along the railroad, is some 550 miles, and does not include any battery or repeating-office. At Cheyenne there was a repeating-office; but as the observatory, as elsewhere stated, was situated west of it, the signals did not work through any repeater; and at the time these observations were made Salt Lake itself was only a relay-office; but there was a switch at Ogden, which had always to be called to "straighten" the wire before the work of exchanging could be commenced. Powerful batteries have always been kept at both places, and lately the repeating-office at Corinne has been removed to Salt Lake, and the latter has not only become a repeating-office but also the headquarters of the western division of the main or eastern branch of the company. 
(9.) Uniform Thabis of The-Reduchions at Rechiving-Station.

SALT LAKE OBSERVATORY, October 5, I872.

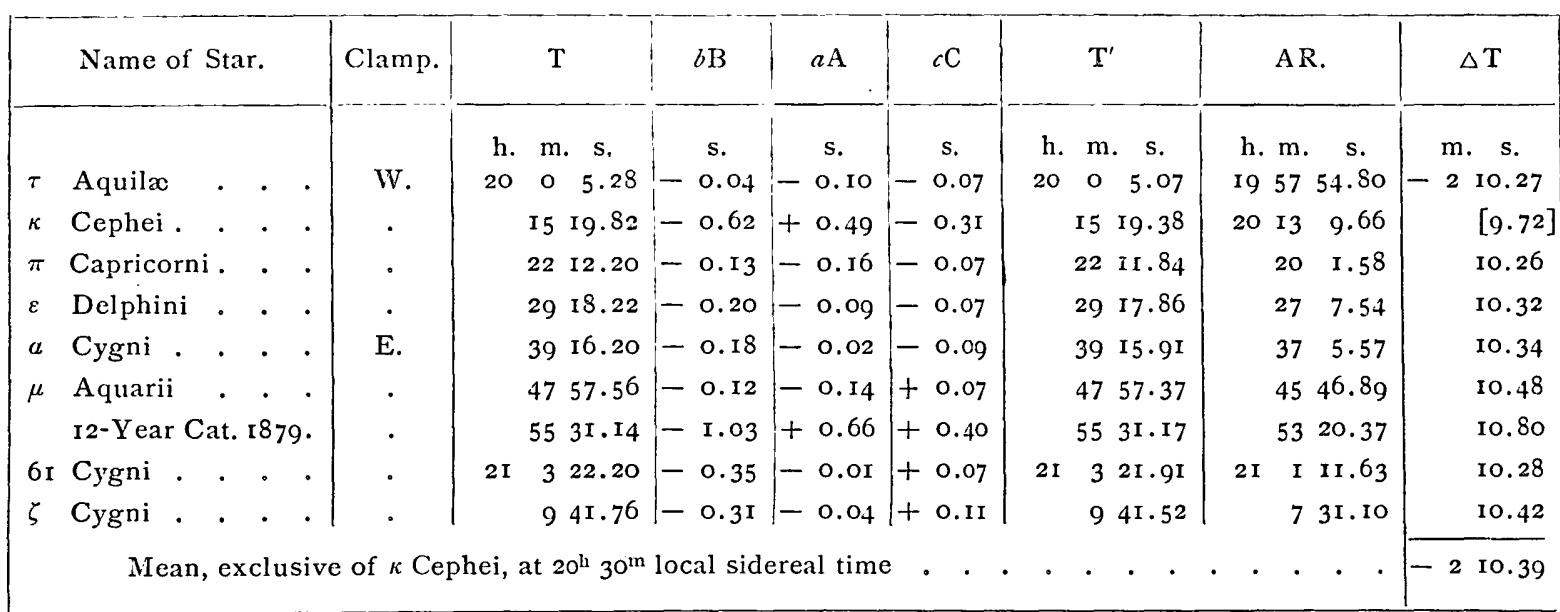

Normal Equations.

$$
\begin{array}{rlrl}
9 \delta t-3.24 a+0.18 c & =-2.22 & & a=-0.18 \\
-3.24 \delta t+22.91 a-10.29 c & =-3.72 & c & =+0.07 \\
0.18 \delta t-10.29 a+63.46 c & =+0.60 & & \delta t=-0.3 \mathrm{I}
\end{array}
$$

SALT LAKE OBSERVATORY, October $5,1872$.

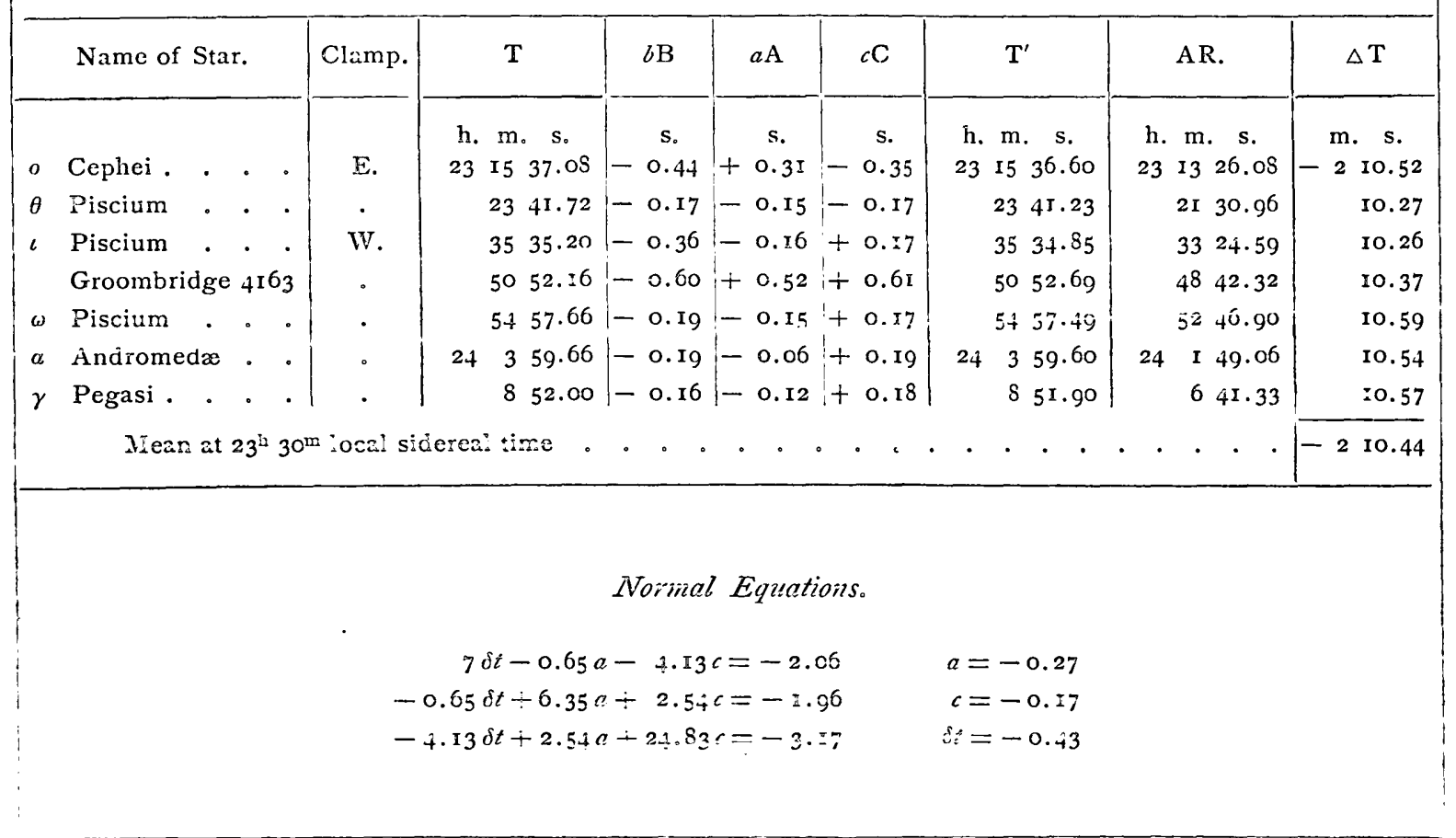


(9.) Uniform Tables of Time-Reductions at Receiving-Station-Continued.

SALT LAKE OBSERVATORY, October 14, I872.

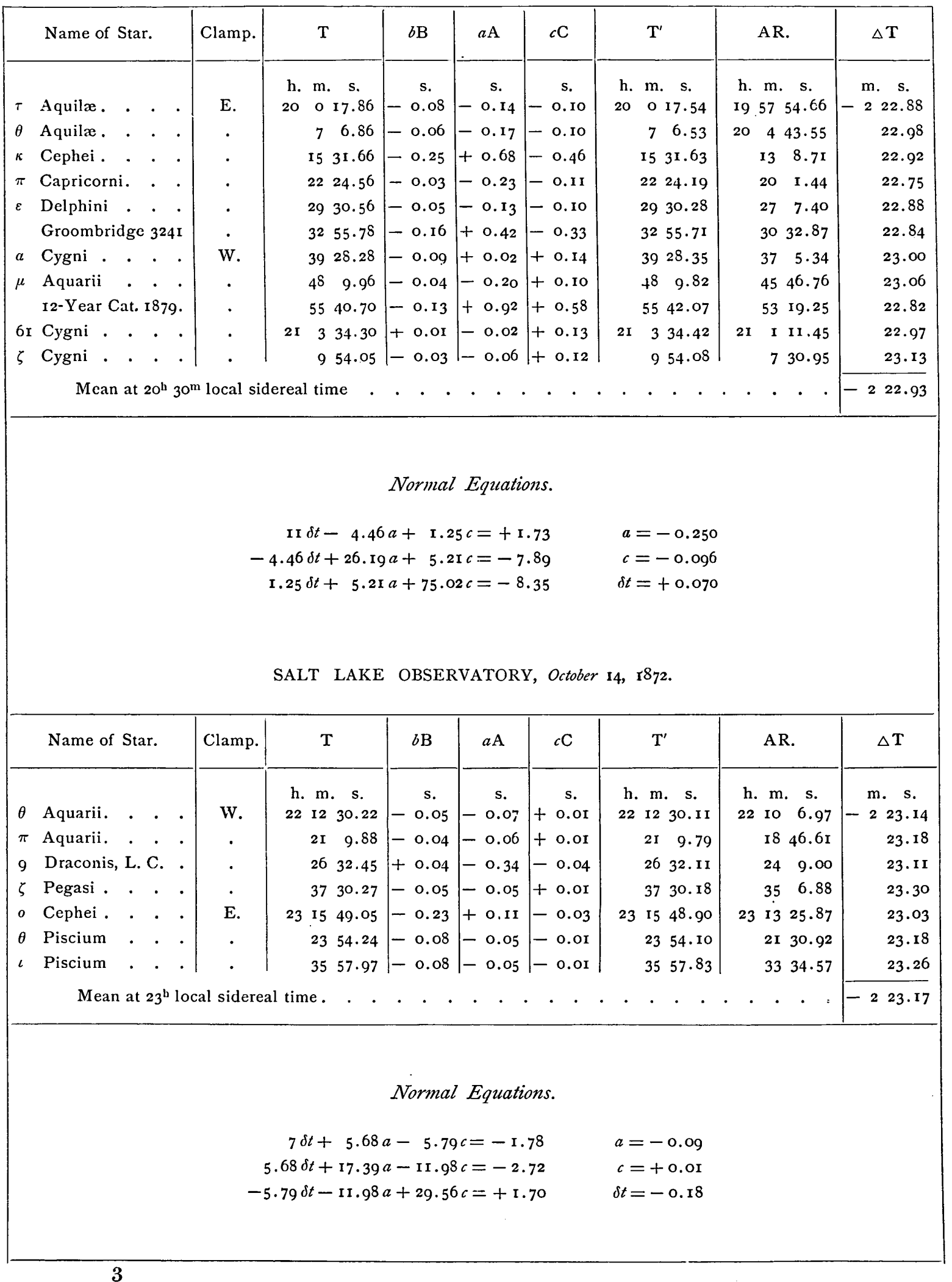


(9.) Uniform Tables of Thue-Reductions at Receiving-Station-Continted.

SALT LAKE OBSERVATORY, October 16, 1872.

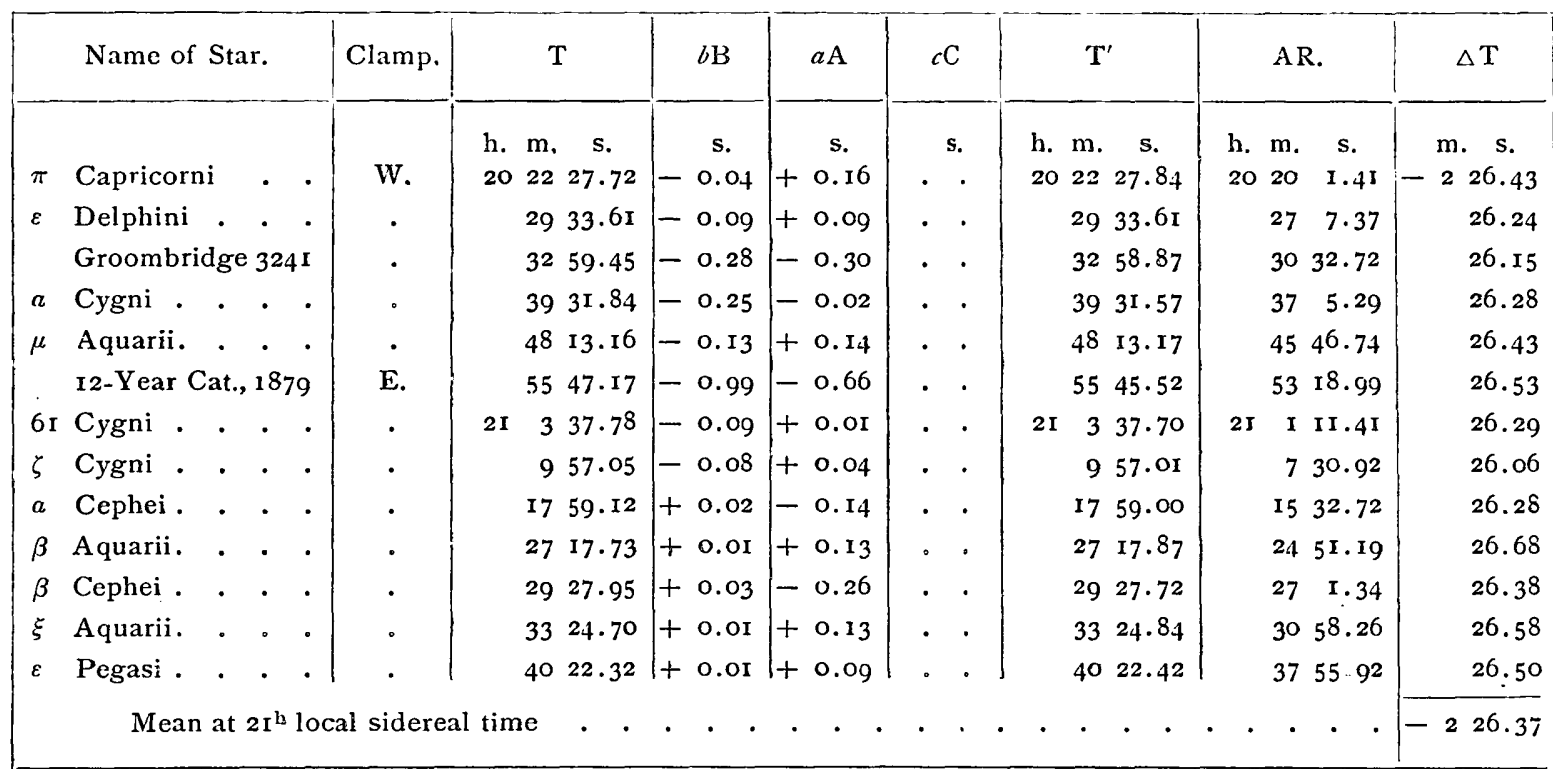

Nornal Equations.

$\begin{array}{rrr}13 \delta t+3.15 a+3.05 c=-5.43 & a=-0.18 \\ 3.15 \delta t+20.33 a+21.20 c=-4.81 & c=0.00 \\ 3.05 \delta t+21.20 a+68.43 c=-4.83 & \delta t=-0.37\end{array}$

SALT LAKE OBSERVATORY, October 16, I872.

\begin{tabular}{|c|c|c|c|c|c|c|c|c|c|c|c|c|}
\hline & Name of Star. & Clamp. & & $\mathrm{T}$ & $b \mathrm{~B}$ & $a \mathrm{~A}$ & $\iota \mathrm{C}$ & \multicolumn{2}{|r|}{$\mathbf{T}^{\prime}$} & \multicolumn{2}{|r|}{ AR. } & $\Delta \mathrm{T}$ \\
\hline$\gamma$ & Cephei . & W. & $\begin{array}{l}\text { h. } \\
23\end{array}$ & $\begin{array}{cc}\text { m. s. } \\
36 & 37.90\end{array}$ & $\begin{array}{c}s . \\
+0.50\end{array}$ & $\begin{array}{l}\text { s. } \\
-0.39\end{array}$ & $\begin{array}{c}\text { s. } \\
+\quad 0.22\end{array}$ & $\begin{array}{l}\text { h. } \\
23\end{array}$ & $\begin{array}{cc}\text { m. s. } \\
36 & 38.23\end{array}$ & $\begin{array}{l}\text { h. } \\
23\end{array}$ & $\begin{array}{l}\text { m. s. } \\
3+\text { II. } 2 f\end{array}$ & $\begin{array}{c}\text { m. s. } \\
-226.99\end{array}$ \\
\hline & Groombridge $4{ }^{1} 63$ & . & & 517.68 & +0.45 & -0.29 & +0.18 & & 518.02 & & $48 \quad 42.09$ & 25.93 \\
\hline$\omega$ & Piscium & . & & $54 \div 3.17$ & +0.12 & +0.09 & +0.05 & & $5+\quad 13.43$ & & 52.46 .80 & 96.57 \\
\hline$a$ & Andromedæ . & - & 24 & $4 \quad 15.36$ & +0.18 & +0.04 & +0.06 & 24 & 415.64 & 24 & I 49.05 & 26.59 \\
\hline$y$ & Pegasi . . . & . & & $9 \quad 7.67$ & $1+0.18$ & +0.07 & +0.05 & & $9 \quad 7.97$ & & $64 \mathrm{~J} \cdot 33$ & 26.64 \\
\hline & Mean at $24^{\text {b }}$ & sicer & . & • & 。 & . & $\circ$ & . & - & . & - & 26.54 \\
\hline
\end{tabular}

Norinal Equations.

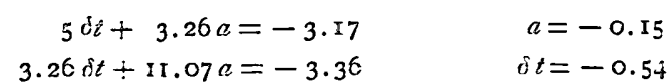




\section{(9.) Uniform Tables of Time-Reductions at Receiving-Station-Continued.}

SALT LAKE OBSERVATORY, October I8, I8j2.

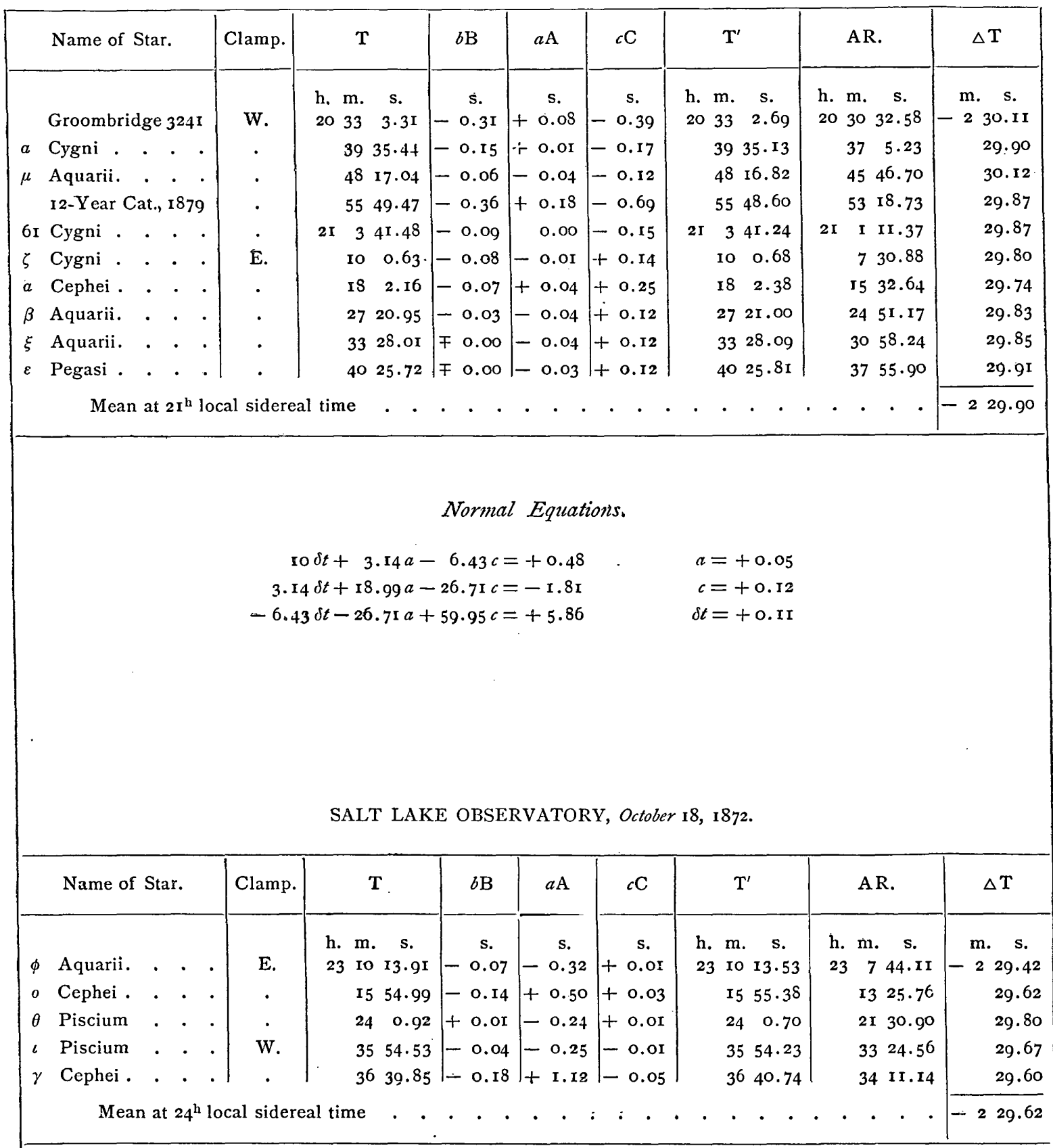

\section{Normal Equations.}

$$
\begin{array}{rlrl}
5 \delta t-\mathrm{I} .85 a-0.78 c & =+2.69 & a & =-0.43 \\
-\mathrm{I} .85 \delta t+9.36 a+9.19 c & =-4.66 & c & =+0.013 \\
-0.78 \delta t+9.19 a+29.27 c & =-3.93 & \delta t+0.38
\end{array}
$$


(9.) Uniform Tables of Time-Reductions at Receiving-Station-Continued.

SALT LAKE OBSERVATORY, October $\mathbf{1 9}, 1872$.

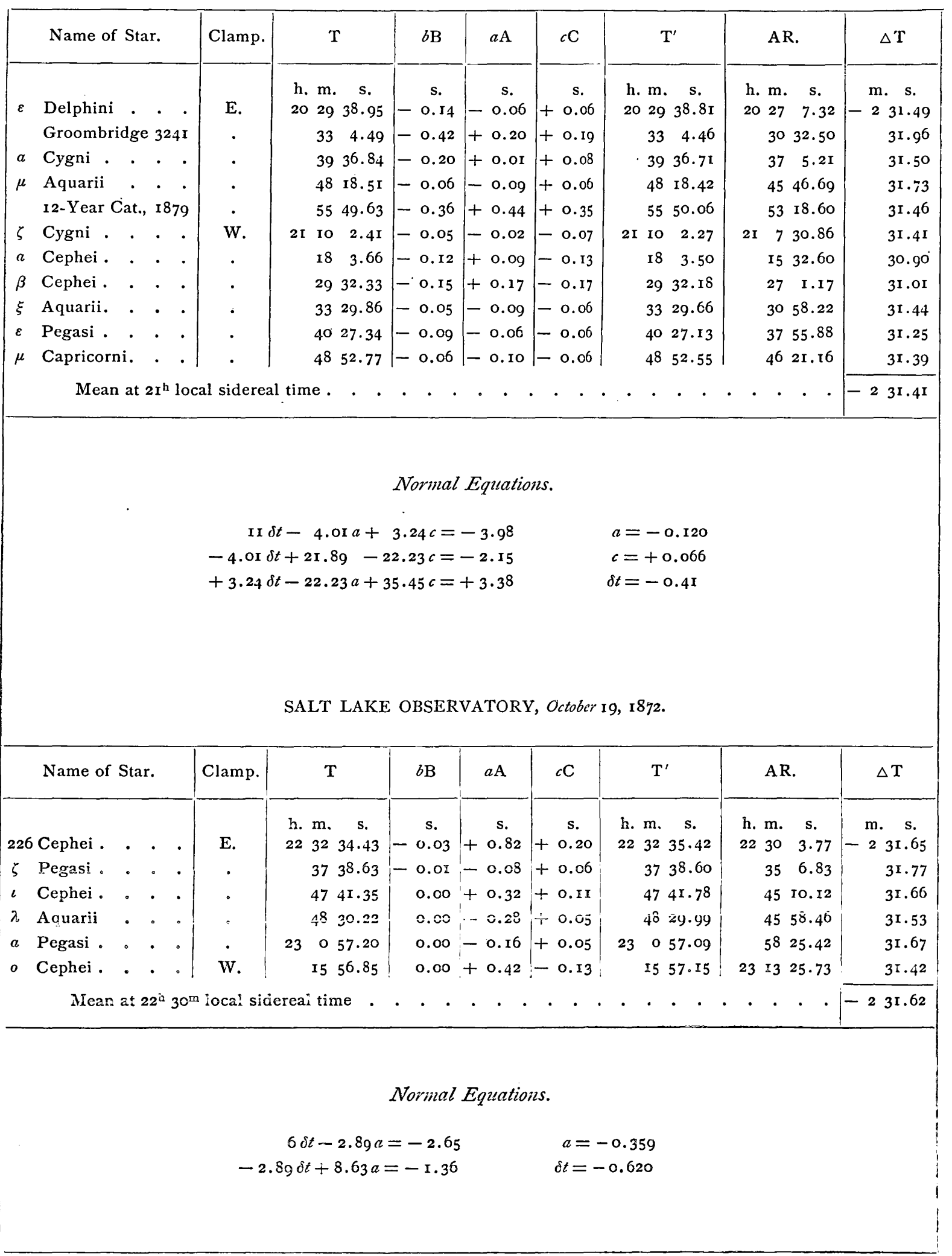


(9.) Uniform Tables of Time-Reductions at Receiving-Station-Continued.

SALT LAKE OBSERVATORY, October $21,1872$.

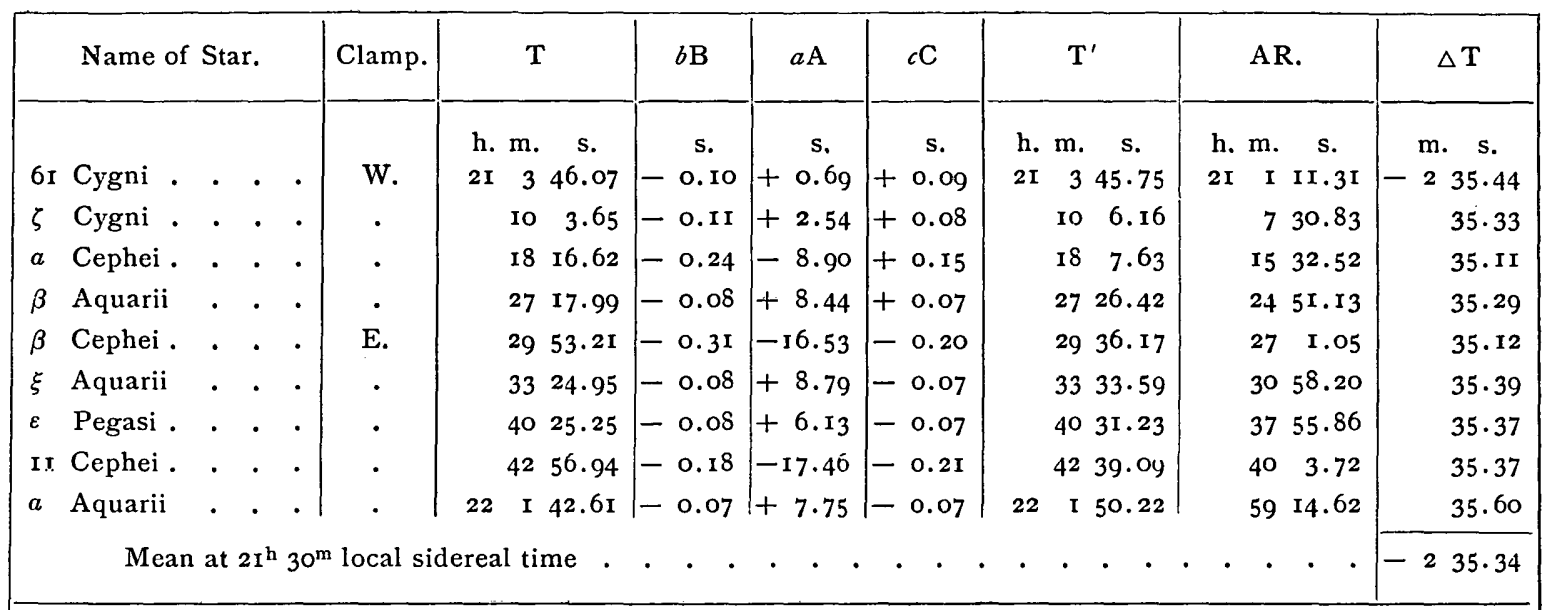

Normal Equations.

$\begin{array}{rlrl}9 \delta t+0.74 a+3.42 c & =-\mathrm{II} .86 & a & =-\mathrm{II} .560 \\ -0.74 \delta t+6.80 a+6.20 c & =-78.82 & c & =-0.072 \\ 3.42 \delta t+6.20 a+29.22 c & =-74.90 & \delta t & =-0.370\end{array}$

SALT LAKE OBSERVATORY, October 21, 1872.

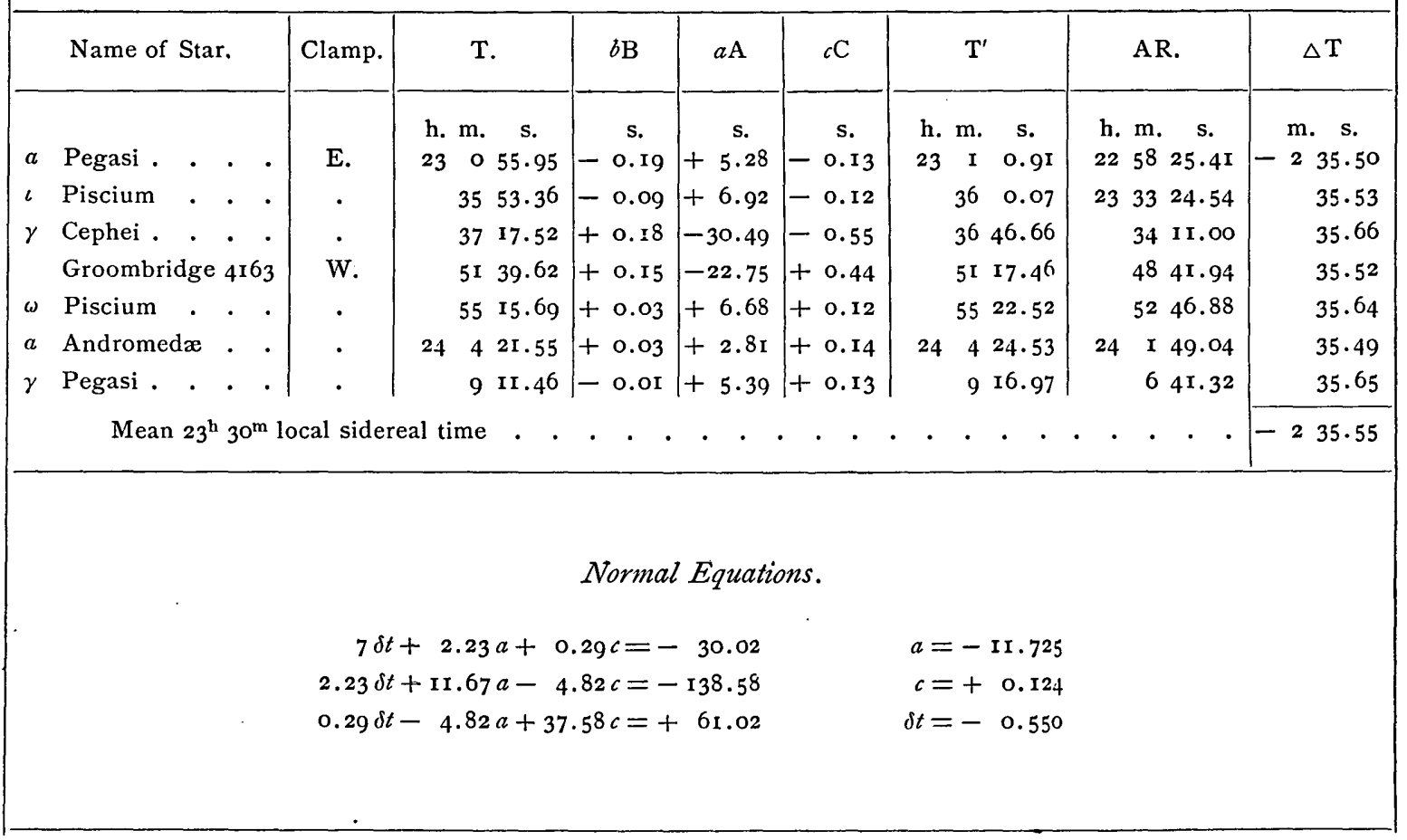


(io.) Uniform Tables of Tme-Reductions at Sexding-Station。

CHEYENNE, WYOMING TERRITORY, October 5, I872.

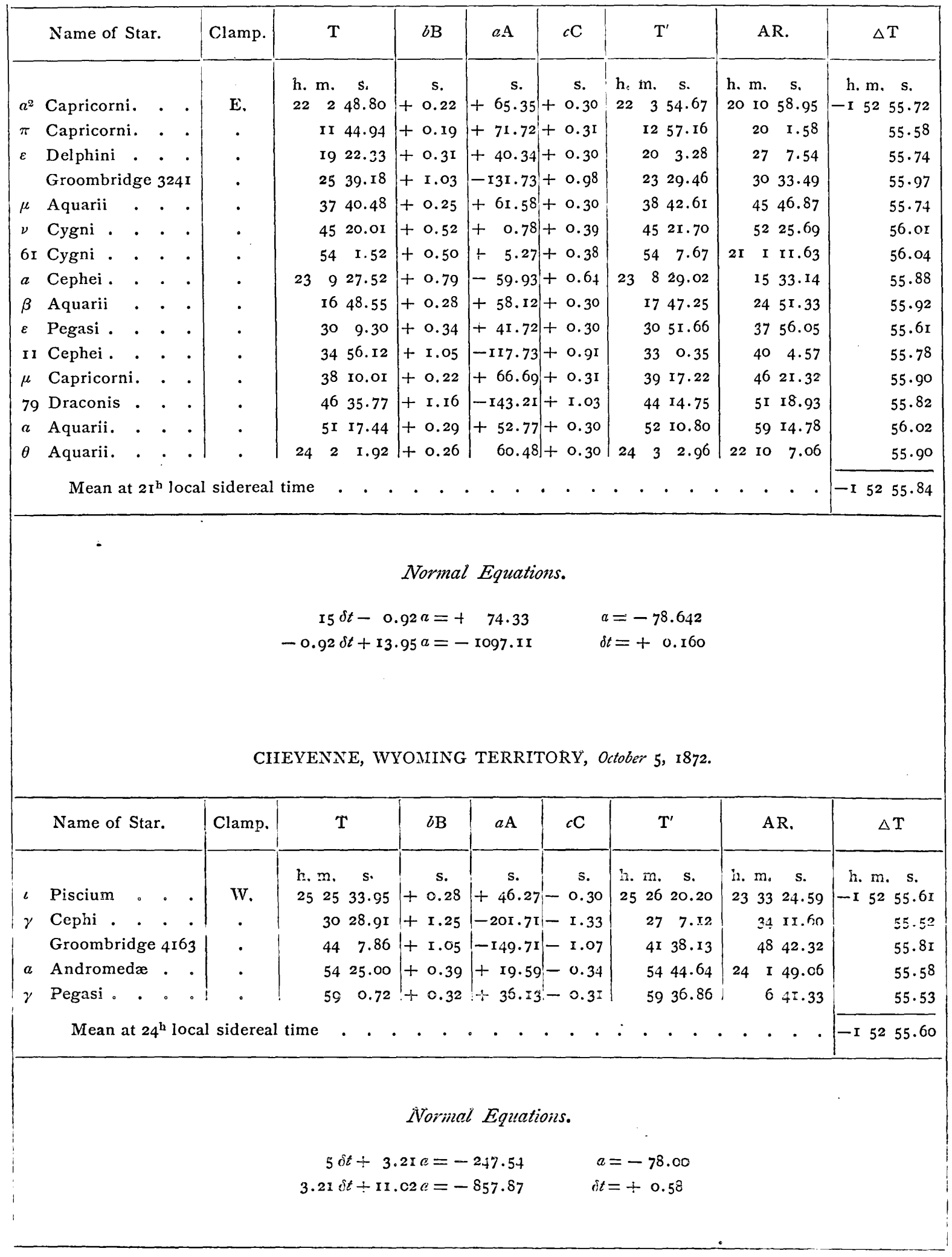


(io.) Uniform Tables of Tme-Reductions at Receiving-Station-Continued.

CHEYENNE, WYOMING TERRITORY, October I4, 1872 .

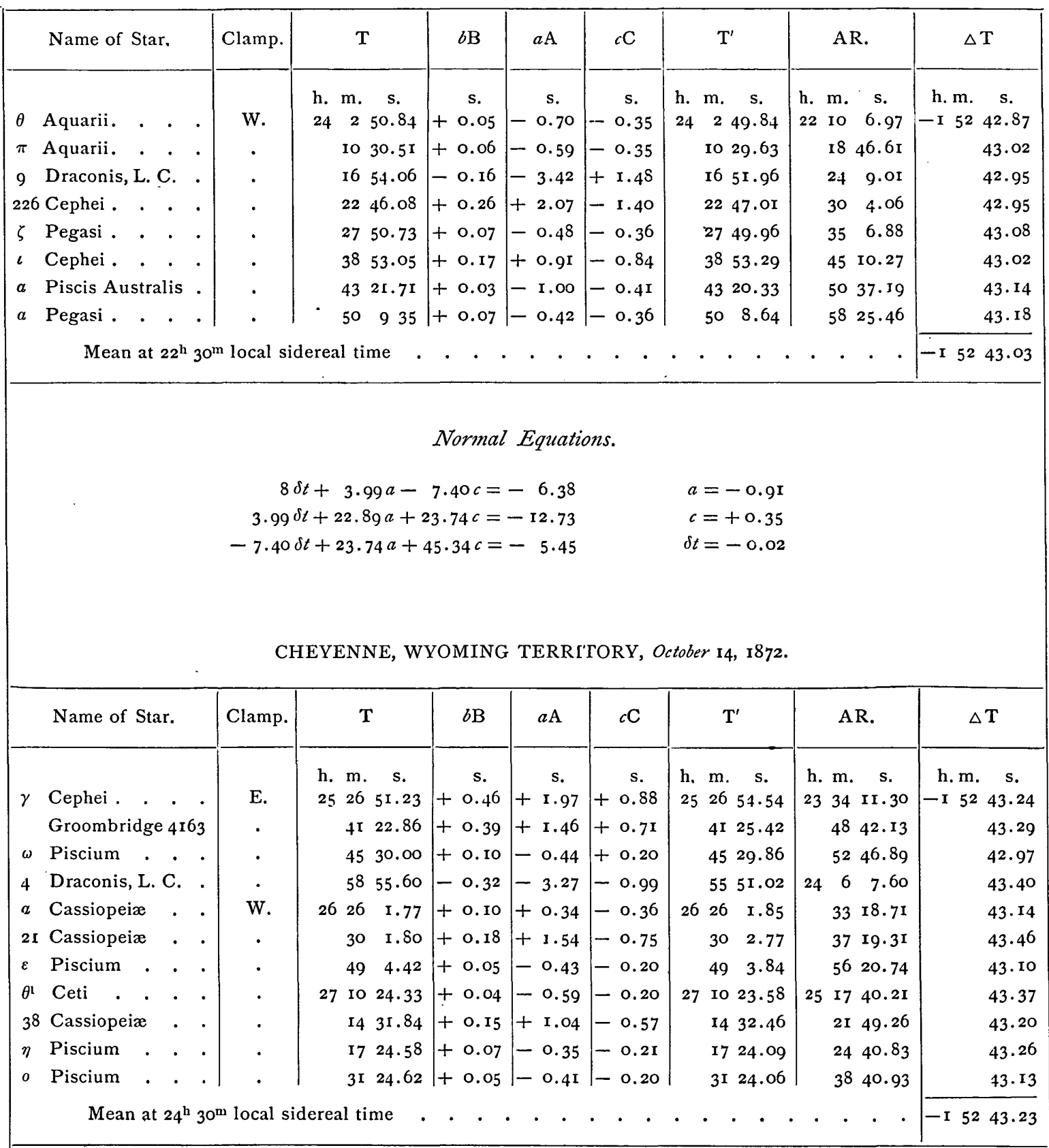

Normal Equations.

$\begin{array}{rlrl}\mathrm{II} \delta t-\mathrm{I} .12 a-8.35 c & =-3.39 & a & =-0.76 \\ -\mathrm{I} .12 \delta t+36.82 a-29.15 c & =-33.68 & c & =+0.20 \\ -8.35 \delta t-29.15 a+86.78 c & =+42.90 & \delta t & =-0.23\end{array}$


(io.) Uniform Tables of Tme-Reductions at Recerving-Station-Continued. CHEYENNE, WYOMING TERRITORY, October 16, 1872.

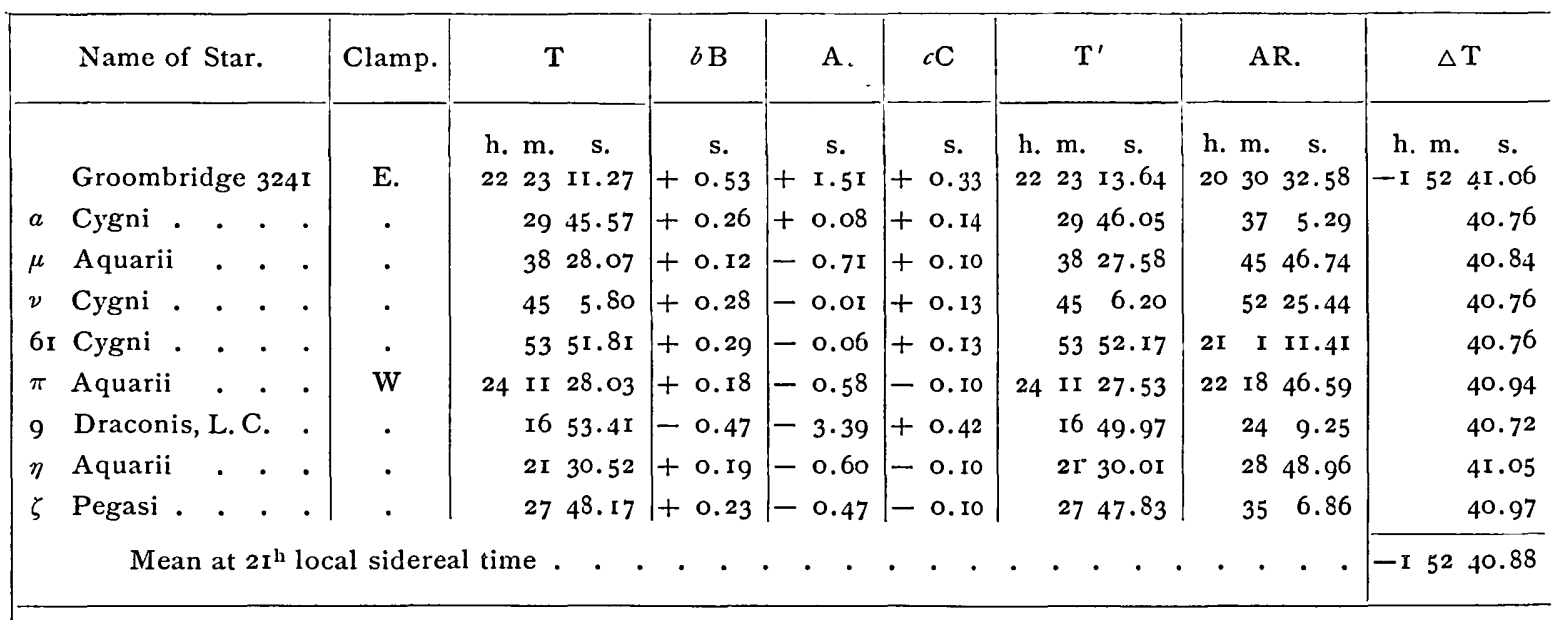

Normal Equations.

$\begin{array}{rl}9 \delta t+4.69 a+9.48 c=-2.42 & a=-0.90 \\ 4.69 \delta t+18.72 a+9.40 c=-15.04 & c=+0.10 \\ 9.48 \delta t+9.40 a+37.94 c=-2.72 & \delta t=+0.10\end{array}$

GHEYENNE, WYOMING TERRITORY, October I6, I872.

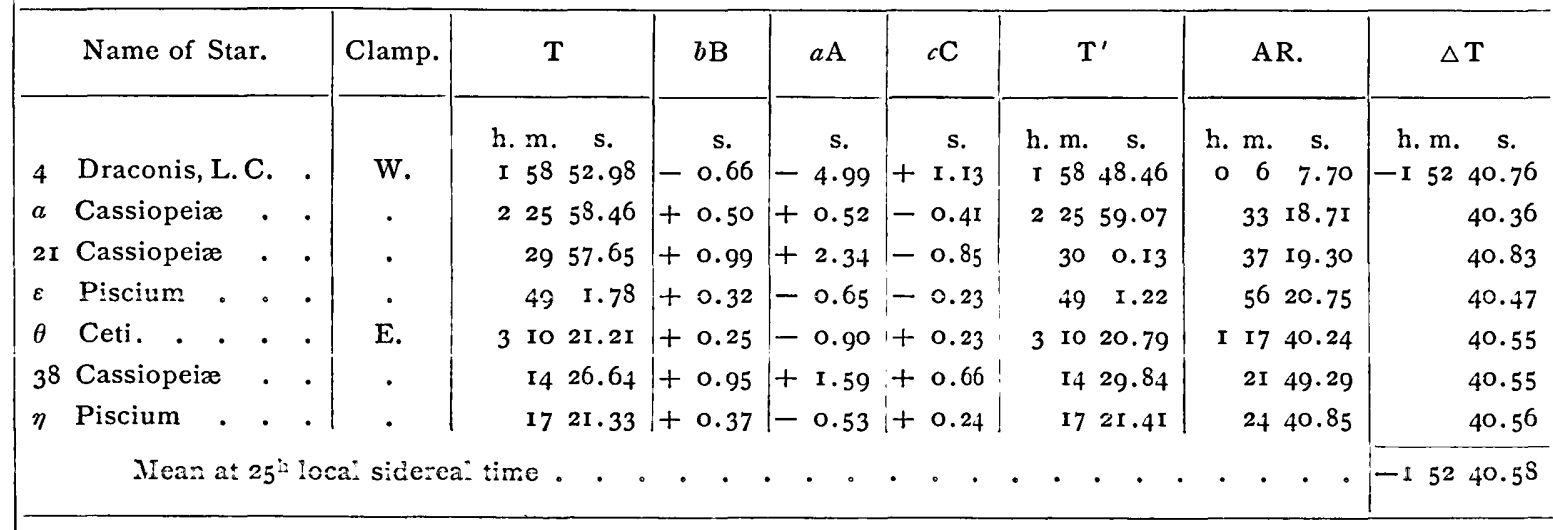

Normal Equations.

$\begin{array}{rlrl}7 \delta t+2.26 a+3.37 c & =-5.92 & a & -\mathrm{I} .160 \\ 2.26 \delta t+25.78+26.25 c & =-25.42 & c & =+0.225 \\ 3.37 \delta t+26.25 a+52.52 c & =-20.7 \mathrm{I} & \delta t & =-0.590\end{array}$


(10.) Uniform Tables of Time-Reductions at Receiving-Station-Continued. CHEYENNE, WYOMING TERRITORY, October $18,1872$.

\begin{tabular}{|c|c|c|c|c|c|c|c|c|c|}
\hline & Name of Star. & Clamp. & $\mathrm{T}$ & $b \mathrm{~B}$ & $a \mathrm{~A}$ & $\iota \mathrm{C}$ & $\mathrm{T}^{\prime}$ & AR. & $\Delta \mathrm{T}$ \\
\hline$\sigma^{2}$ & Ursæ Maj., L.C. & w. & $\begin{array}{lcc}\text { h. m. } & \text { s. } \\
22 & 51 & 48.50\end{array}$ & $\begin{array}{c}\text { s. } \\
-0.14\end{array}$ & $\begin{array}{c}s . \\
-3.13\end{array}$ & $\begin{array}{c}s . \\
+0.52\end{array}$ & 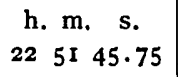 & $\begin{array}{cc}\text { h. m. } & \text { s. } \\
59 & 7.95\end{array}$ & 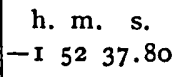 \\
\hline$\zeta$ & Cygni . . . & . & 2309.13 & +0.17 & -0.29 & -0.23 & $\begin{array}{lll}23 & 0 & 8.78\end{array}$ & $21 \quad 7 \quad 30.88$ & 37.90 \\
\hline$a$ & Cephei. . . & . & 89.66 & +0.32 & +0.96 & -0.43 & 810.51 & I5 32.64 & 37.87 \\
\hline $\mathbf{I}$ & Draconis, L. C. & . & II 25.78 & -0.62 & -7.48 & +1.42 & II 19.10 & $918+1.04$ & 38.06 \\
\hline$\beta$ & Cephei. . . & - & 1937.72 & $+0.5 \mathrm{I}$ & +1.78 & -0.59 & I9 39.42 & $\begin{array}{lll}21 & 27 & 1.22\end{array}$ & 38.20 \\
\hline$\xi$ & Aquarii. . & . & 2337.34 & +0.13 & -0.97 & -0.20 & 2336.30 & 3058.24 & 38.06 \\
\hline$\varepsilon$ & Pegasi. . . & . & 3034.49 & +0.17 & -0.67 & -0.20 & 3033.79 & 3755.90 & 37.89 \\
\hline & Cephei. . . & . & 3240.02 & +0.53 & +1.89 & $-0.6 \mathrm{I}$ & 3241.83 & $40 \quad 3.88$ & 37.95 \\
\hline & Capricorni. . & - & $39 \quad 0.4 \mathrm{I}$ & +0.14 & -1.07 & -0.21 & $3^{8} 59.27$ & 4621.17 & 38.10 \\
\hline 79 & Draconis . . & . & 4354.07 & +0.58 & +2.29 & $1-0.69$ & 4356.25 & 5118.18 & 38.07 \\
\hline & Mean at $22^{\mathrm{h}}$ & side & ime . & 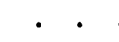 & .. & & . . & . . & -I $52 \quad 37.99$ \\
\hline
\end{tabular}

Normal Equations.

$\begin{array}{rlrl}10 \delta t+5.33 a & =-6.61 & a & =-\mathbf{1} .256 \\ 5.33 \delta t+51.26 a & =-64.32 & \delta t & =+0.010\end{array}$

CHEYENNE, WYOMING TERRITORY, October $\mathbf{1 8}, \mathbf{1 8 7 2 .}$

\begin{tabular}{|c|c|c|c|c|c|c|c|c|c|}
\hline & Name of Star. & Clamp. & $\mathbf{T}$ & $b \mathrm{~B}$ & $a \mathrm{~A}$ & $\iota \mathrm{C}$ & $\mathrm{T}^{\prime}$ & AR. & $\Delta T$ \\
\hline ؛ & Piscium & W. & $\begin{array}{l}\text { h. m. } \\
25 \quad 26 \\
25.90\end{array}$ & $\begin{array}{c}\text { s. } \\
+\quad 0.02\end{array}$ & $\begin{array}{c}\text { s. } \\
-0.5^{8}\end{array}$ & $\begin{array}{c}5 . \\
-0.20\end{array}$ & $\begin{array}{lll}\text { h. } & \text { m. } & \text { s. } \\
25 & 26 & 2.14\end{array}$ & 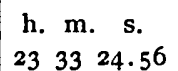 & $\begin{array}{r}\text { h. m. s. } \\
\text {-I } 52 \quad 37.58\end{array}$ \\
\hline & Groombridge ${ }_{41} 6_{3}$ & - & $4 \mathrm{I} \quad 18.63$ & -0.03 & +1.87 & -0.71 & 419.76 & $48 \quad 42.03$ & 37.73 \\
\hline$\omega$ & Piscium $\cdot$. & - & $45 \quad 25.25$ & -0.01 & -0.56 & -0.20 & 4524.48 & $52 \quad 46.89$ & 37.59 \\
\hline 4 & Draconis, L. C. . & - & $5^{8} \quad 48.54$ & +0.10 & $-4 \cdot 19$ & +0.99 & $5845 \cdot 44$ & $24 \quad 6 \quad 7.77$ & 37.67 \\
\hline$a$ & Cassiopeæ. . . & - & 262456.21 & -0.03 & +0.44 & -0.36 & 262456.26 & 3318.70 & 37.56 \\
\hline$\beta$ & Ceti. . . . . & - & $295 \mathrm{I} .3 \mathrm{I}$ & -0.02 & -0.89 & -0.21 & 2950.19 & 3712.55 & 37.64 \\
\hline$\varepsilon$ & Piscium . . & . & $48 \quad 59.06$ & -0.02 & -0.55 & $1-0.20$ & $48 \quad 58.29$ & 5620.76 & 37.53 \\
\hline \multicolumn{4}{|c|}{ Mean at $24^{\mathrm{b}}$ local sidereal time } & . & . & . & . & . & $\begin{array}{lll}-1 & 52 & 37.61\end{array}$ \\
\hline
\end{tabular}

Normal Equations.

$$
\begin{array}{rlrl}
7 \delta t+4.57 a & =-1.76 & a & =-0.975 \\
4.57 \delta t+24.19 a & =-21.82 & \delta t & =+0.390
\end{array}
$$


(io.) Uniform Tables of Time-Reductions at Receiving-Station-Continued. CHEYENNE, WYOMING TERRITORY, October 19, 1872.

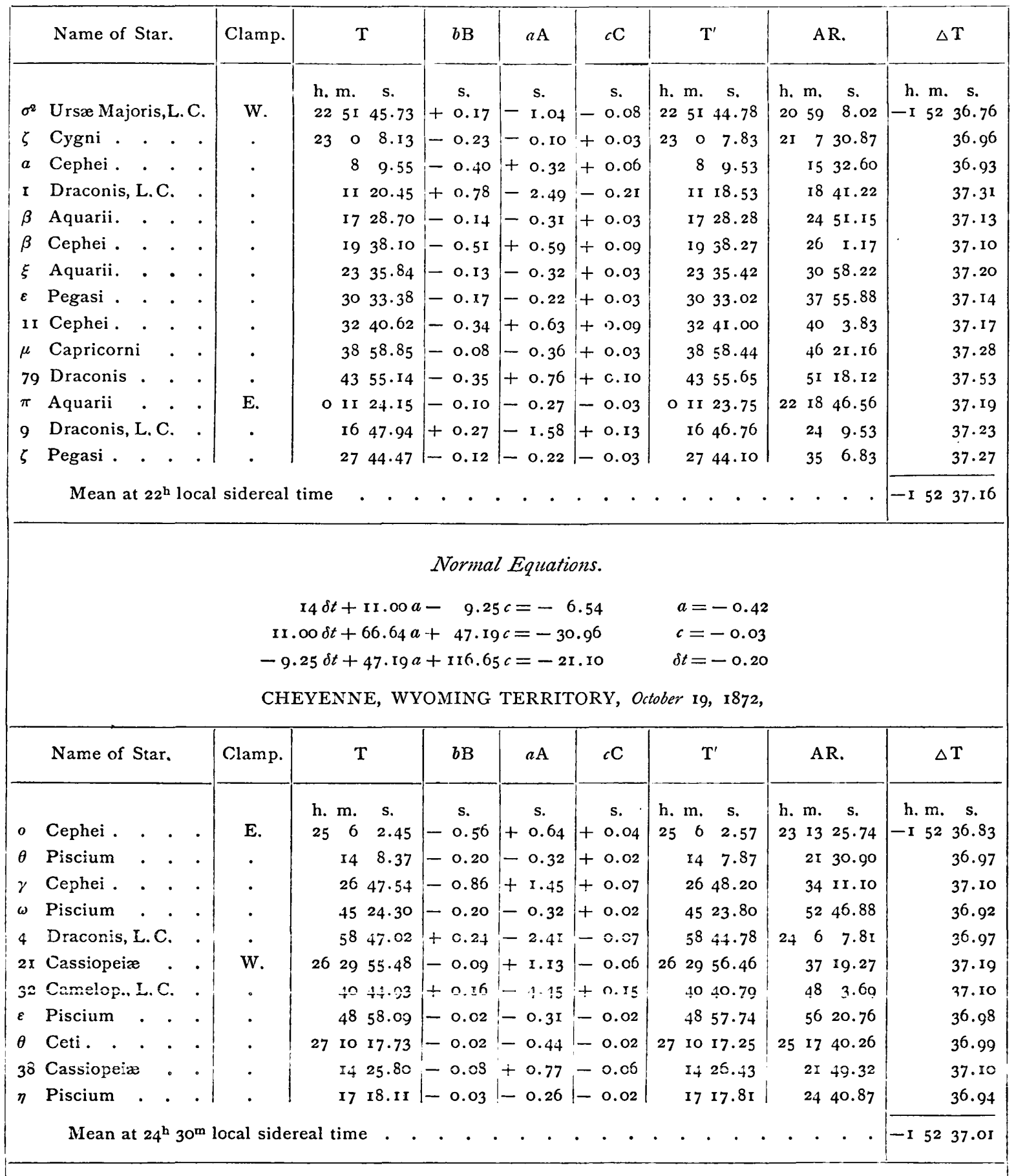

Normal Equations.

$\begin{array}{rlrl}\text { II } \delta t+8.08 a+4.22 c & =-4.52 & a & =-0.560 \\ 8.08 \delta t+97.49 a+52.40 e & =-53.73 & c & =+0.015 \\ 4.22 \delta t+52.40 a+172.36 c & =-26.64 & \delta t & =0.000\end{array}$


(io.) Uniform Tables of Time-Reductions at Receiving-Station-Continued.

CHEYENNE, WYOMING TERRITORY, October 2I, I872.

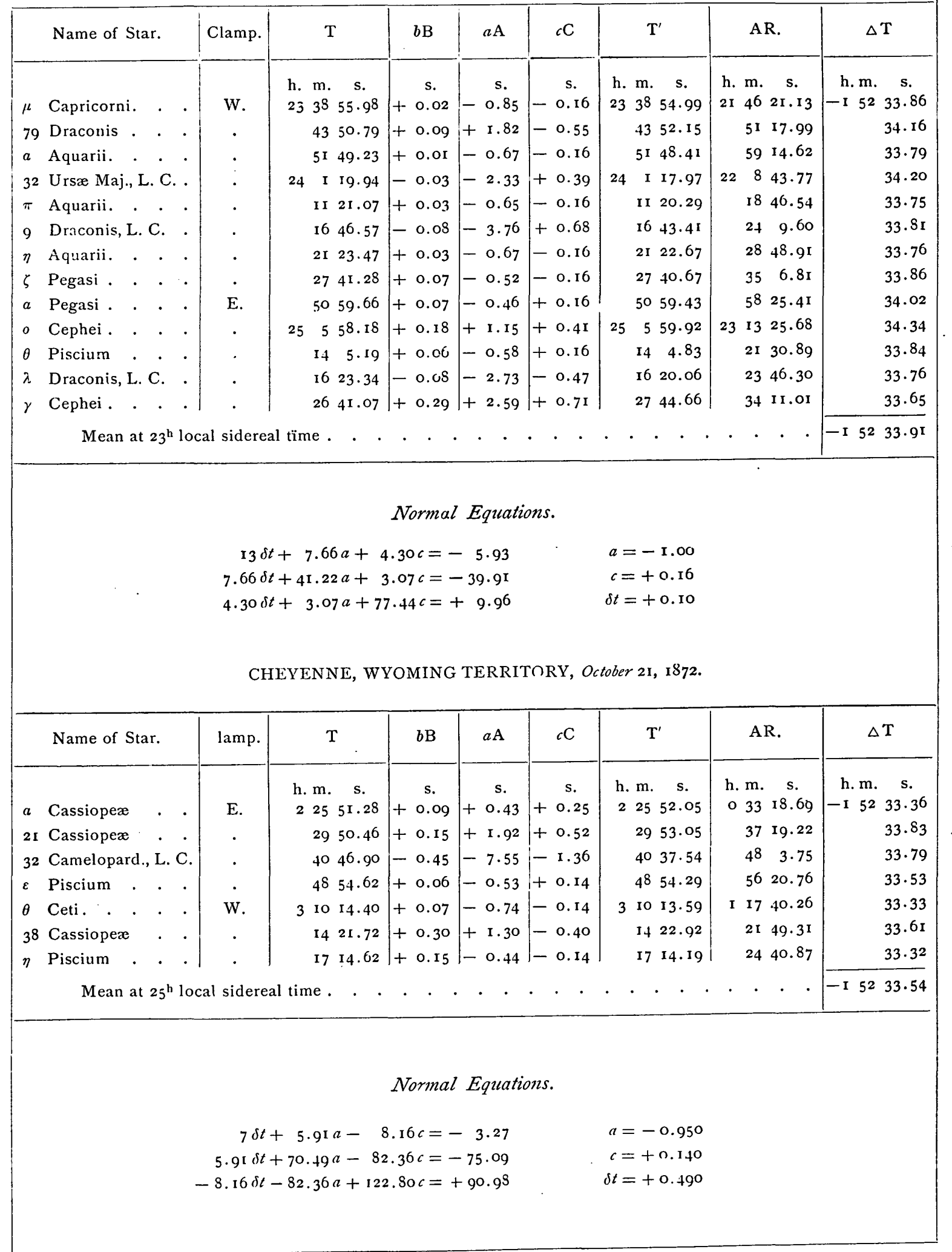


The following table shows the corrections and the rate of the chronometers used at Cheyenne and Salt Lake City:

\section{CHEYENNE.-NEGUS I 499.}

\begin{tabular}{|c|c|c|c|c|}
\hline Date. & $\begin{array}{c}\text { Local } \\
\text { sidereal hour. }\end{array}$ & \multicolumn{2}{|c|}{ Correction of chronometer. } & Rate per hour. \\
\hline $\begin{array}{c}1872 . \\
\text { October } 5\end{array}$ & $\begin{array}{c}\text { h. } \\
22.50\end{array}$ & $\begin{array}{r}\text { h. } \mathrm{m} \\
-\mathrm{I} 52\end{array}$ & $\begin{array}{l}\text { s. } \\
55.725\end{array}$ & \\
\hline 14 & 23.50 & & 43.130 & $+0.05^{8}$ \\
\hline I6 & 23.00 & & 40.725 & $+0.05 \mathrm{I}$ \\
\hline 18 & 23.00 & & 37.800 & $+0.06 \mathrm{I}$ \\
\hline I9 & 23.25 & & 37.085 & +0.030 \\
\hline $2 I$ & 24.00 & & 33.725 & +0.069 \\
\hline
\end{tabular}

SALT LAKE.-NEGUS I 5 I I.

\begin{tabular}{|c|c|c|c|c|}
\hline Date. & $\begin{array}{c}\text { Local } \\
\text { sidereal hour. }\end{array}$ & \multicolumn{2}{|c|}{ Correction of chronometer. } & Rate per hour. \\
\hline $\begin{array}{r}1872 . \\
\text { October }\end{array}$ & $\begin{array}{c}\text { h. } \\
22.00\end{array}$ & $\begin{array}{r}\text { h. } m \\
-02\end{array}$ & $\begin{array}{c}\text { s. } \\
10.415\end{array}$ & \\
\hline 14 & $21 \cdot 75$ & & 23.050 & -0.059 \\
\hline 16 & 22.50 & & 26.455 & -0.070 \\
\hline 18 & 22.50 & & 29.760 & .0 .069 \\
\hline I9 & 21.75 & & $3 \mathrm{I} .5 \mathrm{I} 5$ & -0.075 \\
\hline 2 I & 22.50 & & 35.445 & $-0.08 \mathrm{I}$ \\
\hline
\end{tabular}




\section{(i i.) Grouping of Series of Exchange-Signals.}

This table shows the result obtained for longitude each way each night and the mean of all the nights:

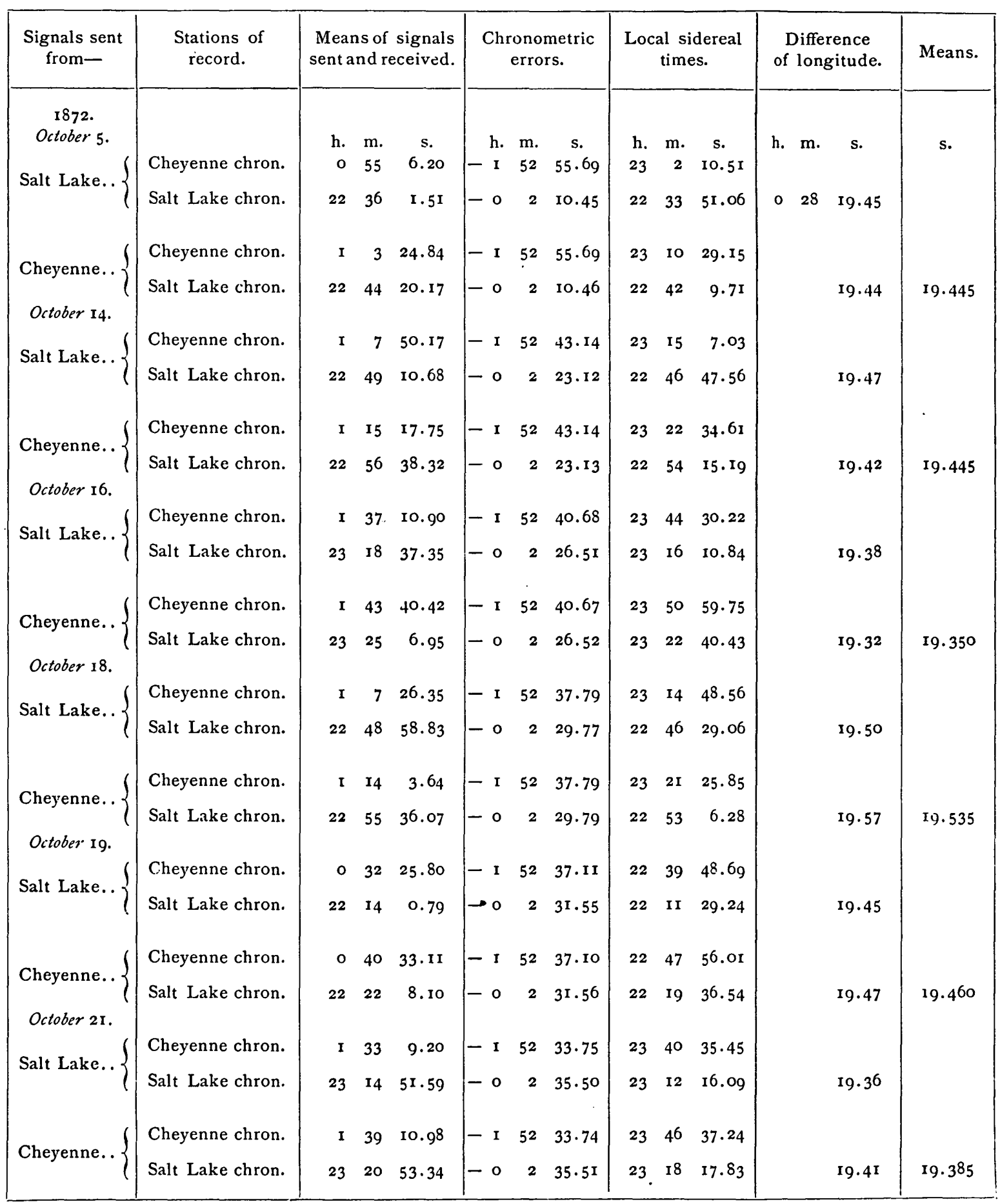

Cheyenne east of Salt Lake City . . . . . . . o $^{\mathrm{h}} 28^{\mathrm{m}} 19^{\mathrm{s}} \cdot 437 \pm 0^{\mathrm{s}}$.017 


\section{(i 2.) Personat Equation.}

Whatever differences there may have been between Mr. Austin and myself in recording time, technically known as the personal equation, is still involved in the longitude. To determine this difference, we had two nights' observations together; the one at Salt Lake in September, 1872, the other at the Naval Observatory, May, 1873. In Salt Lake we used different instruments on the same stars, in Washington the same instrument on different stars. The record, in both instances, was made on a chronograph. The result shows a large difference-Mr. Austin observing later than myself, as may be seen by a statement of the following clock-errors:

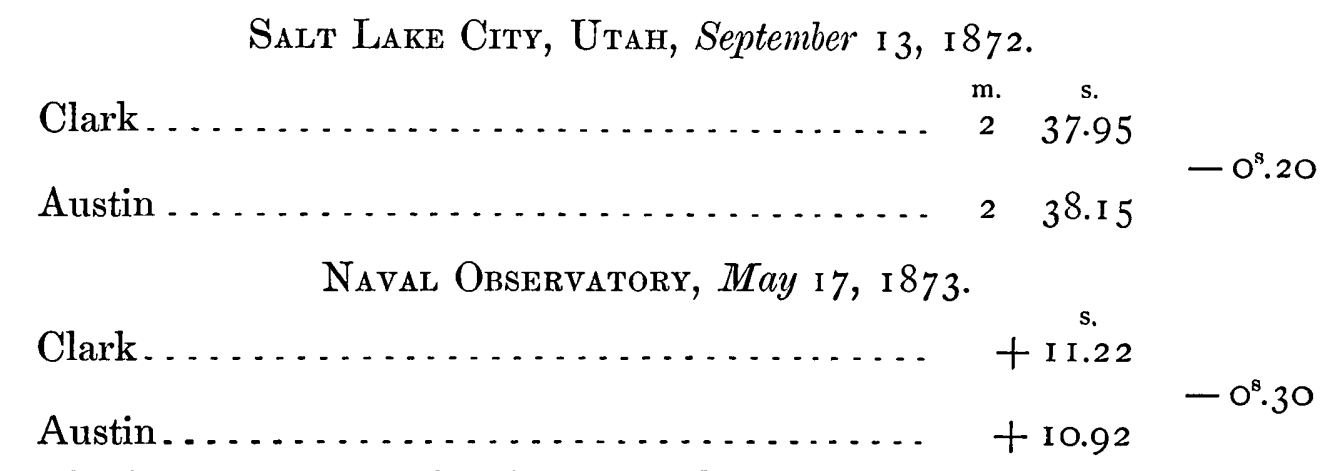

Though this is not considered sufficiently satisfactory to apply without further data, it is positive evidence of the existence of an element affecting our results; and I have the more faith in it because it expresses the sign between us which I would indicate reasoning a priori. I am convinced that; in my own case at least, personal equation is a variable quantity, and introduces an error which cannot be easily eliminated unless some means be devised by which it may be determined in the course of every evening's observations.

\section{(i3.) Probable Error.}

The computations were made by the method of least squares. The conditional equations were sometimes formed with and sometimes without the correction for collimation $(c)$; in the former case it was obtained from a preliminary reduction. All the observations at both stations were made under the same circumstances and conditions, except the night of the $14^{\text {th }}$ at Cheyenne, when the signals had to be received by sound in consequence of the failure of the chronograph to work. It accords with the rest, and is included with full weight; and the provalule error of the final result is by the formula,

$$
\text { Error }=0.6745 \bigwedge \sqrt{\frac{\sum\left(i^{2}\right)}{n(n-1)}}= \pm 0^{\prime \prime} .017 \text {. }
$$

\section{(i4.) Resclitivg Lovgitcde.}

From the foregoing I conclude that the difference of longitude between Salt Lake City observatory and the station at Cheyenne is $28^{\mathrm{m}} 19^{\mathrm{s}} \cdot 437$; which difference, however, is still subject to correction for personal equation; and if any weight is to be given to what is indicated as this equation between $\mathrm{Mr}$. Austin and myself, this longitudinal difference will be reduced by the extent of, perhaps, $\alpha^{s} .2$ of a second. 
(i 5.) Reduction of the Latitude Observations.

Mean Places of Stars for 1872.0 used for Determination of Latitude of Cheyenne, Wyoming Territory.

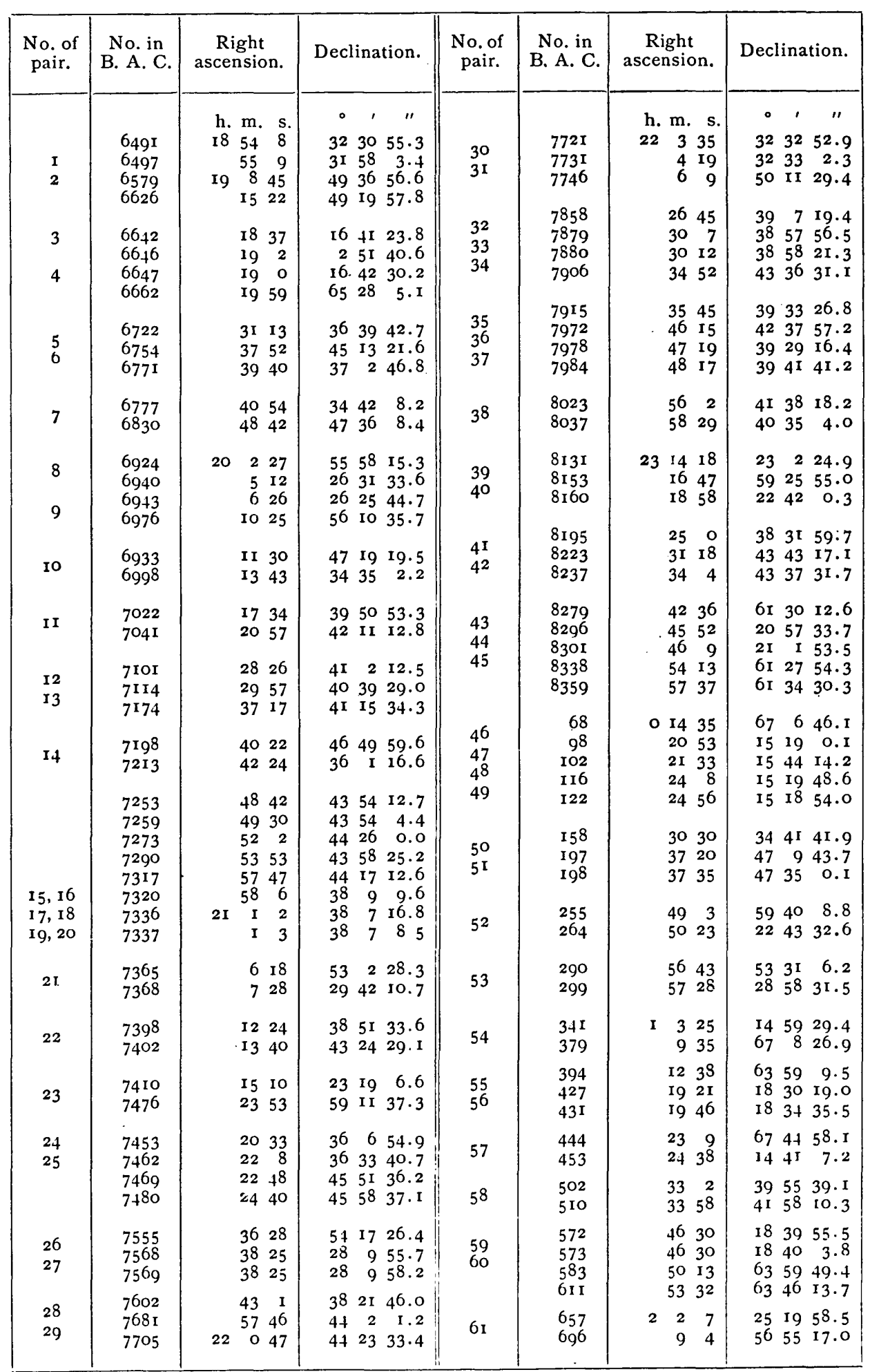


(i 5.) Reduction of the Latitude-Observations-Continued.

Observations for Latitude.-Station, Cheyenne, Wyoming Territory.

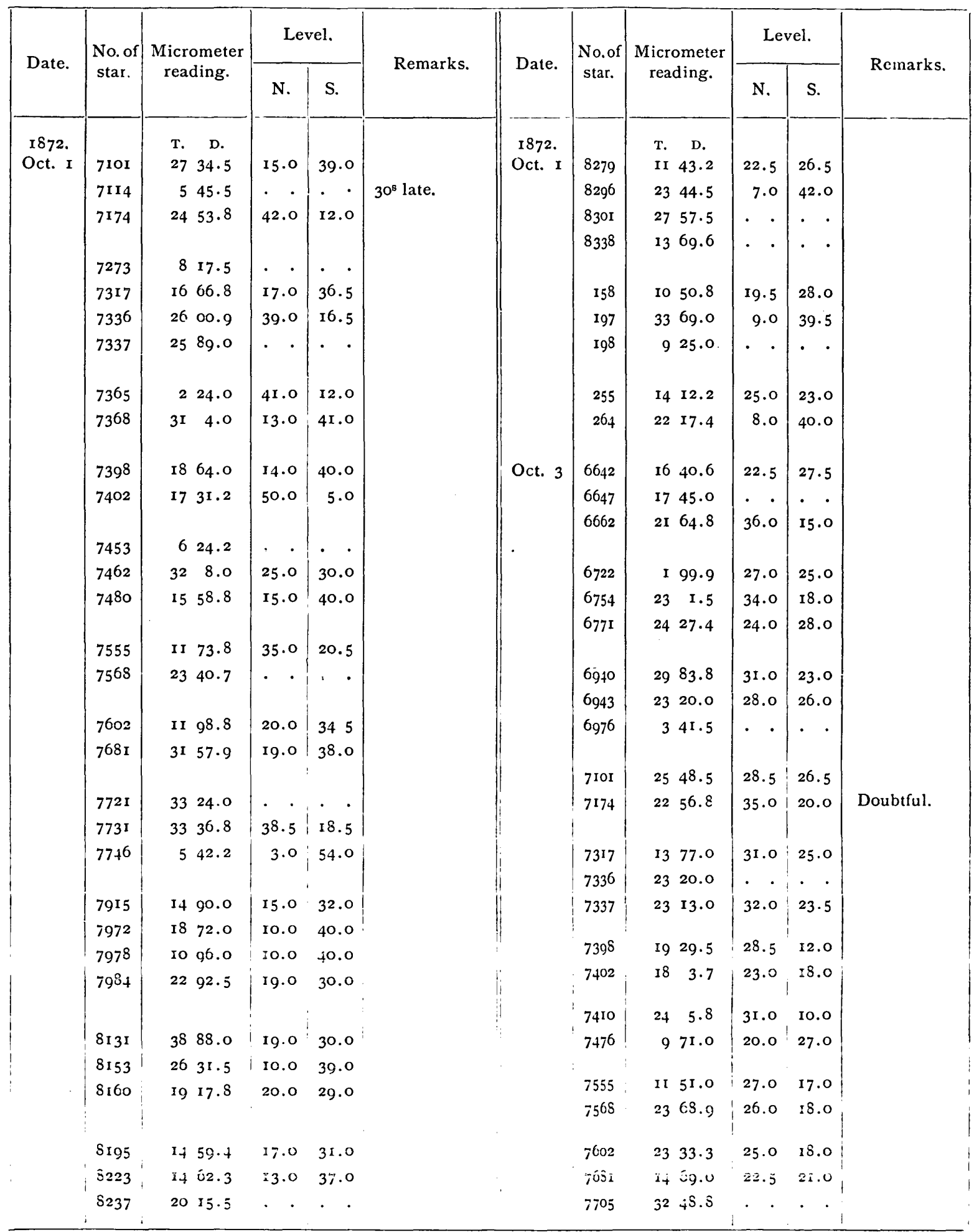


(i5.) Reduction of the Latitude-Observations-Continued.

Observations for Latitude.-Station, Cheyenne, Wyoming Territory.

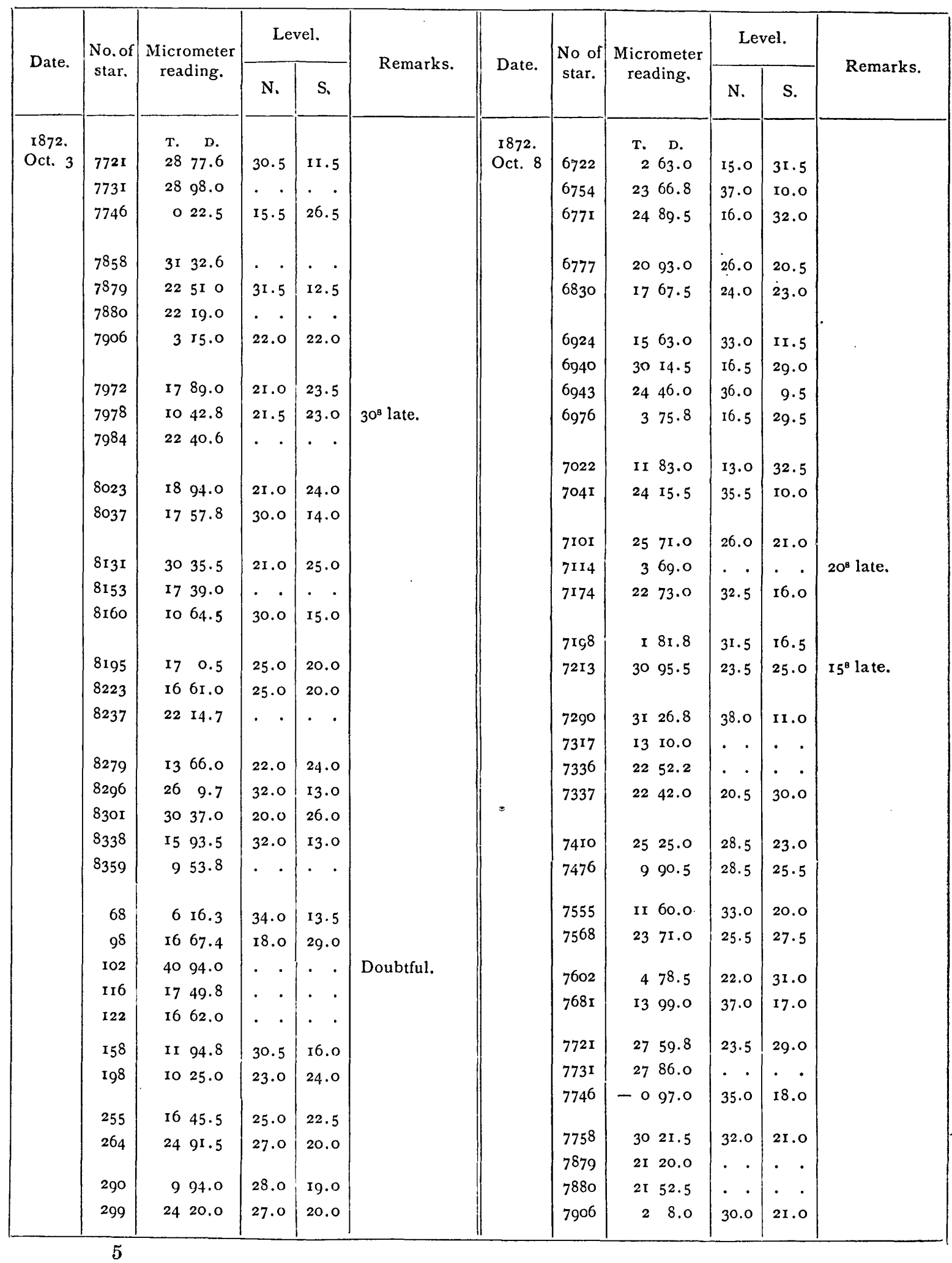


(15.) Reduction of the Latitude-OibSeRvatrons-Continued,

Observations for Latitude._Station, Cheyenne, Wyoming Territory.

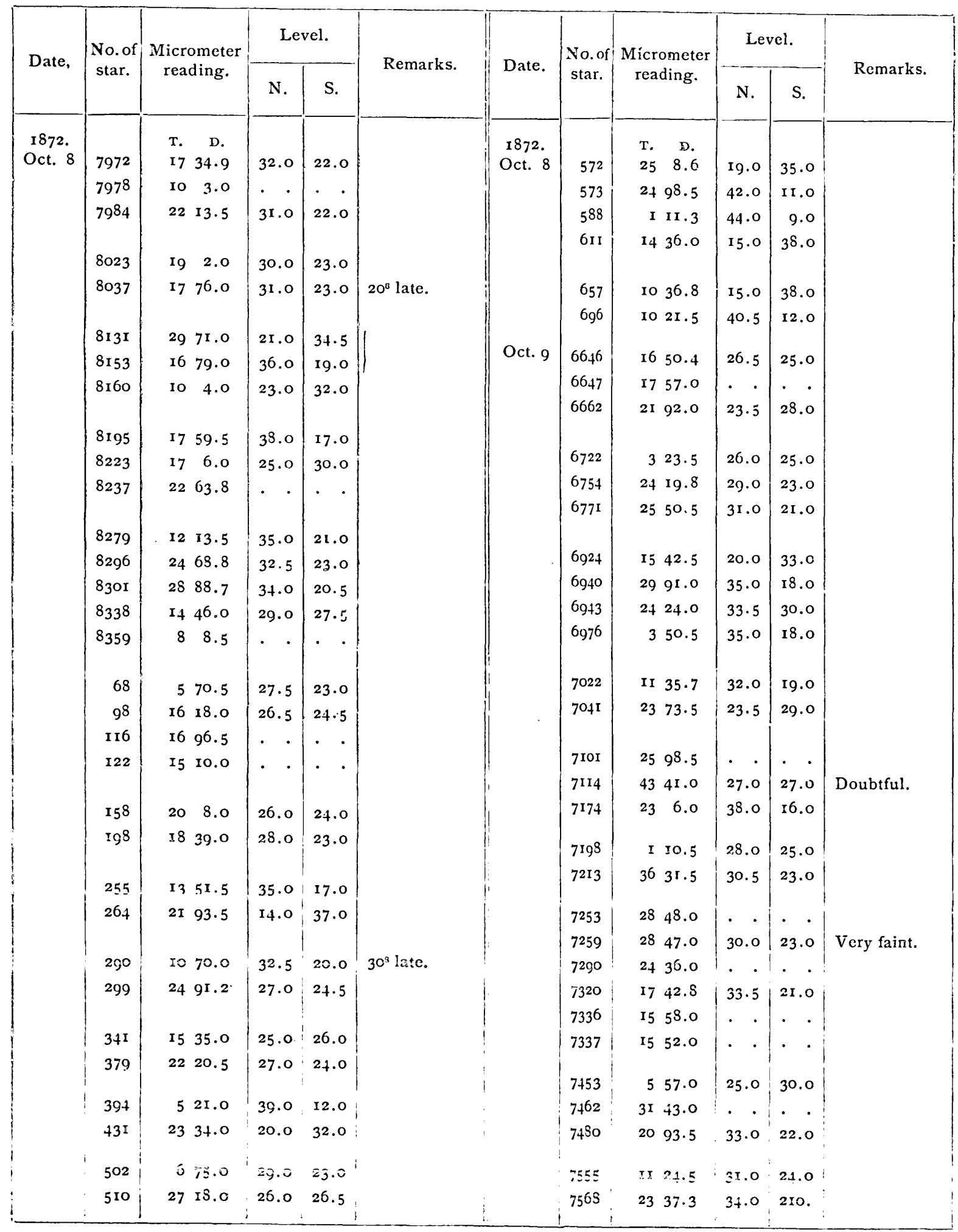


(i5.) Reduction of the Latitude-Observations-Continued.

Observations for Latitude.-Station, Cheyenne, Wyoming Territory.

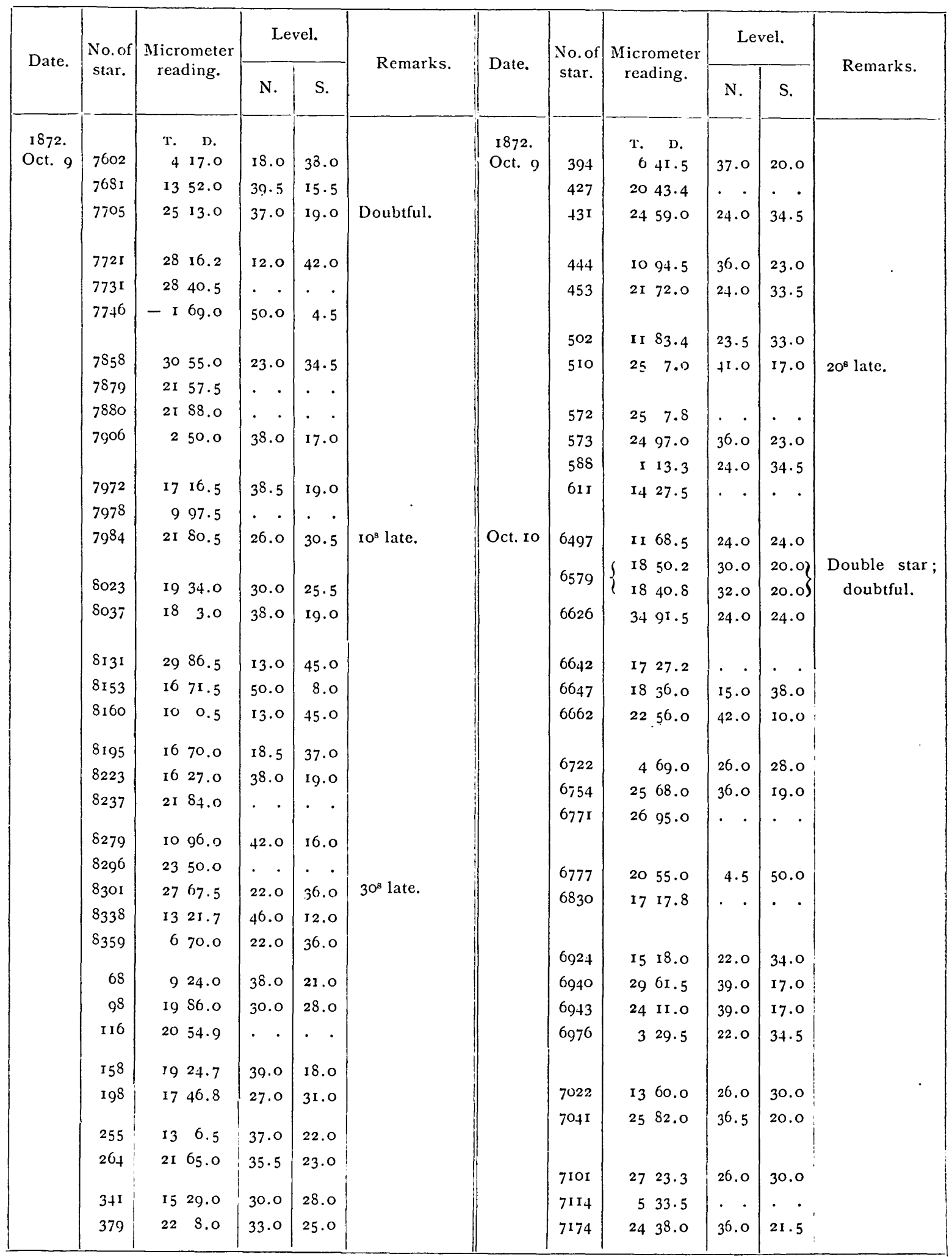


(i5.) Reduction of the Latitude-Omservations-Continued.

Observations for Latitude.-Station, Cheyenne, Wyoming Territory.

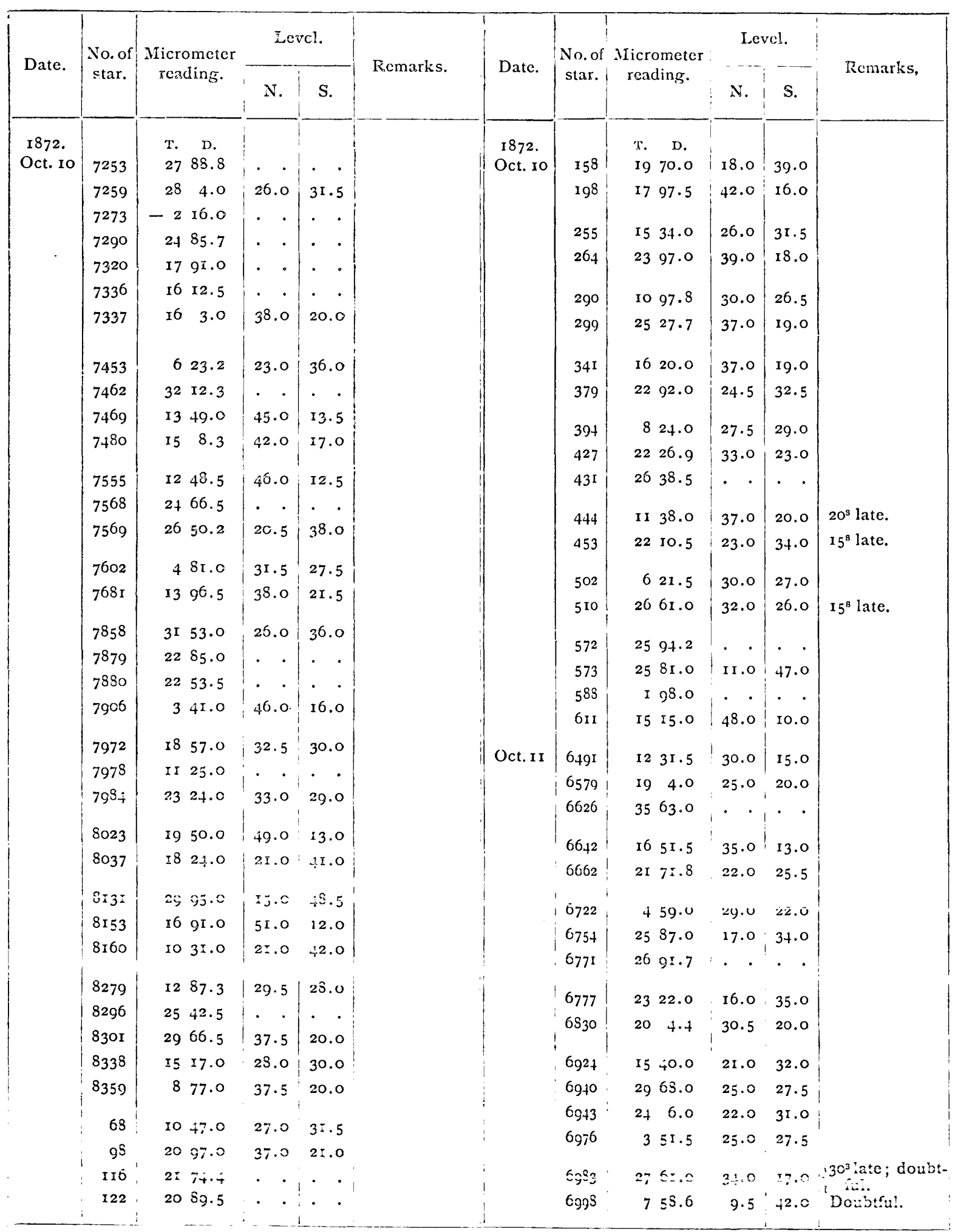


(15.) Reduction of the Latitude-Observations-Continued.

Observations for Latitude.-Station, Cheyenne, Wyoming Territory,

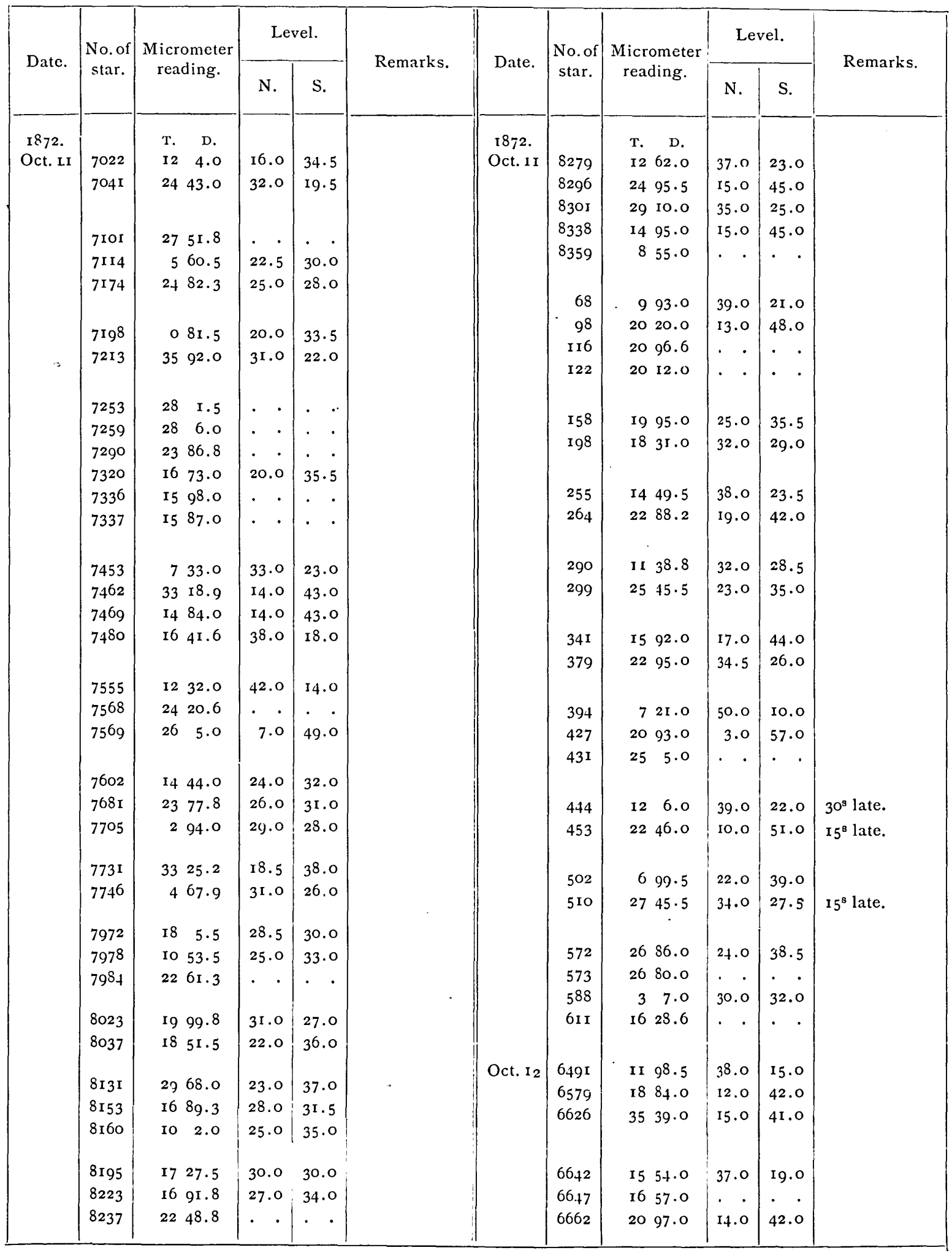


(i 5.) Reduction of the Latitude-Observations-Continued.

Observations for Latitude.-Station, Cheyenne, Wyoming Territory.

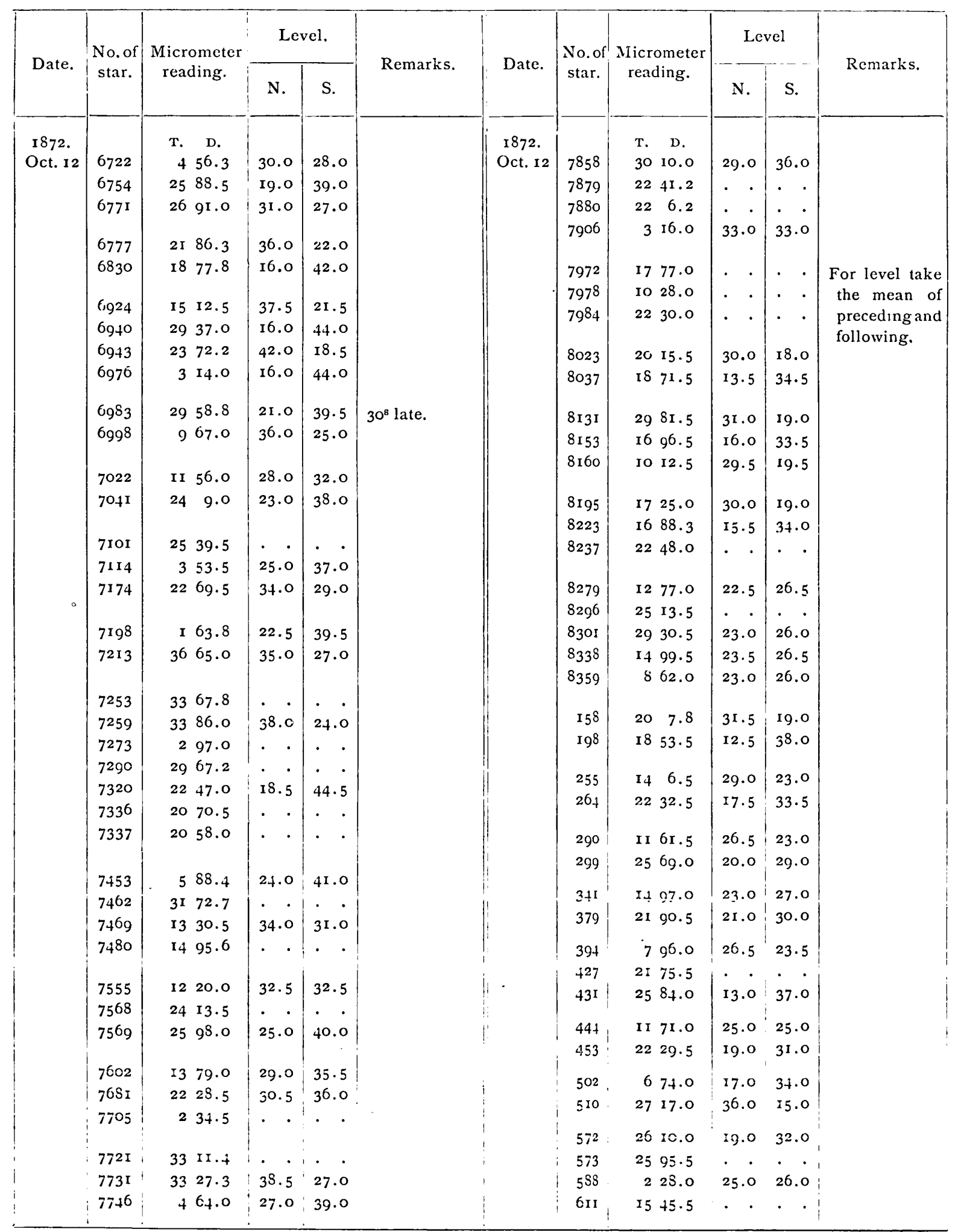


(i 5.) Reduction of the Latitude-Observations-Continued.

Computations for Latitude of Cheyenne, Wyoming Territory.

\begin{tabular}{|c|c|c|c|c|c|c|c|c|c|c|c|}
\hline \multirow{2}{*}{ Date. } & \multirow{2}{*}{$\begin{array}{l}\text { Number } \\
\text { of pair. }\end{array}$} & \multirow{2}{*}{\multicolumn{3}{|c|}{$\begin{array}{l}\text { Half-sum of dec- } \\
\text { linations. }\end{array}$}} & \multicolumn{4}{|c|}{ Corrections. } & \multirow{2}{*}{\multicolumn{3}{|c|}{ Latitude. }} \\
\hline & & & & & \multicolumn{2}{|c|}{ Mic. and ref. } & Level. & \multirow{2}{*}{ Meridian. } & & & \\
\hline \multirow{27}{*}{$\begin{array}{l}1872 . \\
\text { Oct. I }\end{array}$} & & $\circ$ & ' & $"$ & , & $"$ & $"$ & & 。 & , & " \\
\hline & I3 & 40 & 57 & 50.5 & +9 & 53.10 & +1.65 & 0.00 & $4 I$ & 7 & $45 \cdot 3$ \\
\hline & 12 & $4 I$ & 9 & 12.3 & $-\mathbf{I}$ & 27.24 & +1.65 & 0.00 & & & 46.6 \\
\hline & 17 & & 16 & 57.6 & -9 & I 4.28 & +083 & 0.00 & & & 44.1 \\
\hline & 20 & & 12 & 29.8 & -4 & 46,63 & +0.82 & 0.00 & & & 44.0 \\
\hline & $2 \mathrm{I}$ & & & 38.5 & $-I_{4}$ & 55.10 & +0.28 & 0.00 & & & 43.7 \\
\hline & 22 & & 8 & 20.7 & -0 & 41.27 & +5.20 & 0.00 & & & 44.6 \\
\hline & 25 & & 16 & 28.2 & -8 & 32.57 & -8.25 & 0.00 & & & 47.4 \\
\hline & 24 & & 3 & 5.3 & +4 & 50.47 & -8.25 & 0.00 & & & 47.5 \\
\hline & 26 & & 14 & O.I & -6 & 8. 67 & -8.80 & 0.00 & & & 48.6 \\
\hline & $3 \mathbf{I}$ & & 22 & 35.8 & $-\mathrm{I}_{4}$ & 42.54 & -8.50 & 0.00 & & & 44.8 \\
\hline & 30 & & & $3 I . I$ & $-I_{4}$ & 35,77 & -8.50 & 0.00 & & & 46.7 \\
\hline & 35 & & 6 & 0.8 & +1 & 58.72 & $-12.9^{2}$ & 0.00 & & & 46.6 \\
\hline & 36 & & & $55 \cdot 5$ & +4 & I. 18 & - II.22 & 0.00 & & & $45 \cdot 5$ \\
\hline & 37 & & Io & 7.8 & -2 & 10.69 & -11.20 & 0.00 & & & 45.9 \\
\hline & 39 & & I 4 & 27.6 & -6 & 30.52 & -11.00 & 0.00 & & & $46 . \mathrm{I}$ \\
\hline & 40 & & 4 & $15 \cdot 3$ & +3 & 41.82 & -10.50 & 0.00 & & & 46.6 \\
\hline & $4 \mathrm{I}$ & & & 56.2 & +0 & 0.90 & -10.45 & 0.00 & & & 46.6 \\
\hline & 42 & & 5 & $3 \cdot 5$ & +2 & 52.84 & -10.40 & 0.00 & & & 45.9 \\
\hline & 43 & & 14 & 10.0 & -6 & 13.36 & -10.70 & 0.00 & & & 45.9 \\
\hline & 45 & & I5 & 10.4 & -7 & I I $\cdot 36$ & -11.70 & 0.00 & & & $47 \cdot 3$ \\
\hline & 46 & & I3 & 8.4 & -5 & 9.62 & -10.70 & 0.00 & & & 48.1 \\
\hline & 48 & & 13 & 32.6 & -5 & 34.22 & -10.70 & 0.00 & & & 47.7 \\
\hline & 49 & & r3 & $5 \cdot 3$ & -5 & $5 . \mathrm{II}$ & -10.70 & 0.00 & & & 49.5 \\
\hline & 50 & & 55 & 58.3 & +12 & 0.50 & -10.70 & 0.00 & & & 48. I \\
\hline & 51 & & 8 & 36.5 & -0 & 39.10 & - 10.70 & 0.00 & & & 46.7 \\
\hline & 52 & & 12 & 5.4 & -4 & 10.26 & -8.25 & 0.00 & & & 46.8 \\
\hline \multirow[t]{16}{*}{ Oct. } & 3 & $4 \mathrm{r}$ & 5 & 0.0 & +2 & 42.92 & +4.40 & 0.00 & $4 I$ & 7 & $47 \cdot 3$ \\
\hline & 4 & & 5 & 33.2 & +2 & 10.47 & +4.40 & 0.00 & & & $48 . I$ \\
\hline & 5 & 40 & 56 & 49.8 & +10 & 53.18 & +4.95 & 0.00 & & & 47.9 \\
\hline & 6 & $4 \mathrm{I}$ & 8 & 22.0 & -0 & 39.13 & +4.00 & 0.00 & & & 46.9 \\
\hline & 9 & & $2 \mathrm{I}$ & 22.7 & -13 & $4 \mathrm{I} .23$ & +2.80 & 0.00 & & & $44 \cdot 3$ \\
\hline & 9 & & 18 & 28.3 & -10 & 46.02 & +2.80 & 0.00 & & & $45 \cdot I$ \\
\hline & 12 & & 9 & 12.5 & $-I$ & 30.66 & +2.00 & + o. 10 & & & 43.9 \\
\hline & 17 & & 12 & 34.2 & -4 & 53.08 & +3.98 & 0.00 & & & $45 \cdot I$ \\
\hline & I9 & & 12 & 30.0 & -4 & $50.9 \mathrm{r}$ & +3.98 & 0.00 & & & 43. I \\
\hline & 22 & & 8 & 21.0 & -0 & 39.10 & $+5.9 \mathrm{I}$ & 0.00 & & & 47.8 \\
\hline & 23 & & 15 & 40.9 & -7 & 57.04 & +3.85 & 0.00 & & & 47.7 \\
\hline & 26 & & 14 & 0.4 & -6 & 18.52 & +4.95 & 0.00 & & & 46.8 \\
\hline & 28 & & I2 & 13.3 & -7 & 28.62 & +2.33 & 0.00 & & & 47.0 \\
\hline & $3 \mathrm{I}$ & & 22 & 36.3 & -14 & $53 \cdot 71$ & +2.20 & 0.00 & & & 44.8 \\
\hline & $3 \mathrm{I}$ & & 22 & 30.8 & $-I_{4}$ & $47 \cdot 36$ & +2.20 & 0.00 & & & 45.6 \\
\hline & 32 & & 22 & I5.0 & $-\mathrm{I}_{4}$ & $35 \cdot 7 \mathrm{I}$ & +5.20 & 0.00 & & & 44.5 \\
\hline
\end{tabular}


(15.) Reidction of the Latitude-Obshrvations-Continued.

Computations for Latitude of Cheyenne, Wyoming Territory.

\begin{tabular}{|c|c|c|c|c|c|c|c|c|c|c|c|}
\hline \multirow{2}{*}{ Date. } & \multirow{2}{*}{$\begin{array}{l}\text { Number } \\
\text { of pair. }\end{array}$} & \multirow{2}{*}{\multicolumn{3}{|c|}{$\begin{array}{l}\text { Half-sum of dec- } \\
\text { linations. }\end{array}$}} & \multicolumn{5}{|c|}{ Corrections. } & \multirow{2}{*}{\multicolumn{2}{|c|}{ Latitude. }} \\
\hline & & & & & \multicolumn{2}{|c|}{ Mic. and ref. } & \multicolumn{2}{|c|}{ Level. } & \multirow{2}{*}{$\frac{\text { Meridian. }}{\prime \prime}$} & & \\
\hline \multirow{20}{*}{$\begin{array}{l}1872 . \\
\text { Oct. }\end{array}$} & & 。 & ' & " & , & $"$ & & $"$ & & ० , & $"$ \\
\hline & 33 & $4 I$ & 17 & $45 \cdot 9$ & - I0 & $1.7 x$ & + & 5.20 & 0.00 & $4 \mathrm{I} \quad 7$ & 49.4 \\
\hline & 34 & & 17 & 33.5 & -9 & 51.76 & + & 5.50 & 0.00 & & 47.2 \\
\hline & 36 & & & $55 \cdot 9$ & +3 & $5 \mathrm{I} .9 \mathrm{I}$ & - & I. 10 & 0.00 & & 46.7 \\
\hline & 37 & & IO & 8.4 & -2 & 20.36 & - & 1.10 & 0.00 & & 46.9 \\
\hline & 38 & & 7 & 0.3 & +0 & 42.33 & + & 3.57 & 0.00 & & 46.2 \\
\hline & 39 & & 14 & 28.0 & -6 & 42.95 & + & 3.10 & 0.00 & & 48.2 \\
\hline & 40 & & 4 & $15 \cdot 7$ & +3 & 29.63 & + & 2.00 & 0.00 & & $47 \cdot 3$ \\
\hline & $4 \mathrm{I}$ & & 7 & 56.6 & -0 & 12.28 & + & 2.75 & 0.00 & & 47.1 \\
\hline & 42 & & 5 & 3.9 & +2 & $39.8 \mathrm{I}$ & + & 2.70 & 0.00 & & 46.4 \\
\hline & 43 & & 14 & I0. 4 & -6 & 26.54 & + & .4 .67 & 0.00 & & 48.6 \\
\hline & 44 & & I5 & 10.8 & -7 & 28.64 & + & 3.57 & 0.00 & & $45 \cdot 7$ \\
\hline & 45 & & 18 & 28.8 & - Io & 47.46 & + & 3.60 & 0.00 & & 44.9 \\
\hline & 46 & & I3 & 9.0 & -5 & 26.68 & + & 2.60 & 0.00 & & 44.9 \\
\hline & 47 & & 25 & $45 \cdot 9$ & -18 & 0.87 & + & 2.60 & 0.00 & & 47.6 \\
\hline & 48 & & I3 & $33 . I$ & -5 & 52.29 & + & 2.60 & 0.00 & & 43.4 \\
\hline & 49 & & I3 & 5.8 & -5 & 25.00 & + & 2.60 & 0.00 & & 43.4 \\
\hline & 50 & & 8 & 37.0 & -0 & 52.77 & + & $3.7 \mathrm{I}$ & 0.00 & & 47.9 \\
\hline & 52 & & I 2 & 5.8 & -4 & 22.94 & + & 2.60 & 0.00 & & $45 \cdot 5$ \\
\hline & 53 & & 15 & $4 \cdot 4$ & -7 & 23.20 & + & 4.40 & 0.00 & & 45.6 \\
\hline \multirow[t]{23}{*}{ Oct. } & 5 & 40 & 56 & 49.9 & +10 & 53.86 & + & 2.88 & 0.00 & $\begin{array}{ll}4 \mathrm{I} & 7\end{array}$ & 46.6 \\
\hline & 6 & $4 I$ & 8 & 22.2 & -0 & 38.13 & + & 3.60 & 0.00 & & 47.7 \\
\hline & 7 & & 9 & 26.4 & $-I$ & $4 \mathrm{I} \cdot \mathrm{I} 7$ & + & 1.78 & 0.00 & & 47.0 \\
\hline & 8 & & I 5 & 12.7 & -7 & $3 \pi .13$ & + & 2.47 & 0.00 & & 44.0 \\
\hline & 9 & & I 8 & 28.6 & - IO & 43.42 & + & $3.7 \mathrm{I}$ & 0.00 & & 48.9 \\
\hline & II & & $\mathbf{I}$ & 22.3 & +6 & 23.06 & + & 1.65 & 0.00 & & 47.0 \\
\hline & I2 & & 9 & 13.0 & $-\mathbf{I}$ & 32.62 & + & $5.9 \mathbf{I}$ & 0.00 & & 46.3 \\
\hline & 13 & 40 & 57 & $5 \mathrm{I} \cdot 3$ & +9 & $51 \cdot 76$ & + & 6.20 & 0.00 & & $49 \cdot 3$ \\
\hline & 17 & $4 I$ & 3 & 11.1 & +4 & 31.83 & + & 4.80 & 0.00 & & 47.7 \\
\hline & 20 & & 12 & 30.7 & -4 & 49.67 & + & 4.80 & 0.00 & & 45.8 \\
\hline & 23 & & I5 & 41.6 & -7 & 56.92 & + & 2.30 & 0.00 & & 47.0 \\
\hline & 26 & & $\mathrm{I}_{4}$ & I. 2 & -6 & I6.38 & + & 3.00 & 0.00 & & 47.8 \\
\hline & 30 & & 22 & 36.3 & -14 & 56.04 & + & 3.30 & $0 . c 0$ & & 43.6 \\
\hline & $3 I$ & & 22 & 31.6 & $-\mathrm{I}_{4}$ & 47.90 & + & 3.30 & 0.00 & & 47.0 \\
\hline & 33 & & I7 & 34.5 & -9 & 54.25 & + & 5.50 & 0.00 & & 45.8 \\
\hline & 34 & & 17 & 46.9 & - 10 & $4 \cdot 35$ & + & $5 \cdot 30$ & 0.00 & & 47.9 \\
\hline & 36 & & 3 & 56.5 & +3 & 47.47 & + & $5 \cdot 30$ & 0.00 & & 49.3 \\
\hline & 37 & & 10 & 9.5 & -2 & $2 S .75$ & + & 5.20 & $0 . c 0$ & & 46.0 \\
\hline & 39 & & 7 & I. 2 & $+c$ & 39.16 & + & 4.50 & $0 . c 0$ & & 44.5 \\
\hline & 39 & & $I_{4}$ & 29.1 & -6 & $4 \mathrm{~T} .55$ & + & 1.00 & $0 . c 0$ & & 48.5 \\
\hline & 40 & 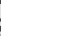 & 4 & 16.9 & $\div 3$ & 29.79 & - & 0.33 & o.co & & 46.4 \\
\hline & 41 & & 7 & 57.8 & -0 & 16.63 & + & 4.40 & 0.00 & & 45.6 \\
\hline & 42 & & 5 & $5 \cdot I$ & +2 & 36.74 & + & 4.40 & $0 . c 0$ & & 46.2 \\
\hline
\end{tabular}


(15.) Reduction of tue Latitude-Observations-Continued。

Computations for Latitude of Cheyenne, Wyoming Territory.

\begin{tabular}{|c|c|c|c|c|c|c|c|c|c|c|c|c|c|}
\hline \multirow{2}{*}{ Date. } & \multirow{3}{*}{$\begin{array}{l}\text { Number } \\
\text { of pair. }\end{array}$} & \multirow{2}{*}{\multicolumn{3}{|c|}{$\begin{array}{l}\text { Half-sum of dec- } \\
\text { linations. }\end{array}$}} & \multicolumn{6}{|c|}{ Corrections. } & \multirow{2}{*}{\multicolumn{3}{|c|}{ Latitude. }} \\
\hline & & & & & Mic. & nd ref. & & evel. & $\mathrm{Me}$ & eridian. & & & \\
\hline \multirow{16}{*}{$\begin{array}{l}\text { is } 82 . \\
\text { Bct. } 8\end{array}$} & & . & , & " & , & " & & $"$ & & $" 1$ & 。 & , & 11 \\
\hline & 43 & $4 I$ & 14 & II .5 & -6 & 30.15 & + & 6.50 & & 0.00 & 48 & 7 & 47.8 \\
\hline & 47 & & & $\$ 2.0$ & -7 & 59.49 & + & 3.90 & & 0.00 & & & 46.4 \\
\hline & 45 & & 18 & 30.0 & - I0 & 46.53 & + & 3.10 & & 0.00 & & & 46.6 \\
\hline & .46 & & 13 & 10.2 & -5 & 25.56 & + & I. 80 & & 0.60 & & & 46.4 \\
\hline & .48 & & 13 & $34 \cdot 4$ & -5 & $49 \cdot 9^{6}$ & + & 1. 80 & & 0.00 & & & 46.2 \\
\hline & .49 & & 13 & $7 . \mathrm{I}$ & -5 & 23.10 & + & I. 80 & & 0.00 & & & 45.8 \\
\hline & 51 & & 8 & $3 S .2$ & -0 & $52 \cdot 52$ & $\frac{1}{4}$ & 2.00 & & 0.00 & & & $47 \cdot 7$ \\
\hline & 52 & & 12 & 7.0 & -4 & 21.69 & - & I. 37 & & 0.00 & & & 43.9 \\
\hline & 53 & & I 5 & $5 \cdot 2$ & -7 & 21.71 & + & 4. IO & + & 0.10 & & & $47 \cdot 7$ \\
\hline & 54 & & 4 & $23 \cdot 3$ & +3 & 33.05 & + & 0.55 & & 0.00 & & & 46.9 \\
\hline & 5.5 & & $\times 7$ & 7.6 & - 9 & $23 \cdot 4^{8}$ & + & 4.10 & & 0.00 & & & 48.2 \\
\hline & 58 & & 57 & I I $\cdot 3$ & + Jo & $34 \cdot 9^{6}$ & + & 1.50 & & 0.00 & & & 47.8 \\
\hline & 59 & & 20 & $5 \cdot 7$ & -12 & 25.08 & + & 5.20 & & 0.00 & & & 45.8 \\
\hline & 60 & & 13 & 17.8 & -5 & 30.22 & & 2.20 & & 0.00 & & & 49.8 \\
\hline & $6 I$ & & 7 & 50.3 & -0 & 4.75 & + & I. 50 & & 0.00 & & & 47.1 \\
\hline \multirow[t]{27}{*}{ Oct. 9} & 4 & $4 \pi$ & 5 & $33 \cdot 3$ & +2 & $\mathrm{I} 5.20$ & - & 0.90 & & 0.00 & $4 I$ & 7 & 47.6 \\
\hline & 5 & 40 & $5^{6}$ & $49 \cdot 9$ & $+1 n$ & $5 \mathrm{I} \cdot 53$ & + & 2.10 & & 0.00 & & & $43 \cdot 5$ \\
\hline & 6 & $4 I$ & .8 & 22.2 & -0 & 40.62 & + & $5 \cdot 50$ & & 0.00 & & & $47 \cdot I$ \\
\hline & 8 & & 15 & 12.8 & -7 & 30.19 & + & I. 10 & & 0.00 & & & $43 \cdot 7$ \\
\hline & 9 & & 18 & 28.7 & -10 & 47.44 & & 2.90 & & 0.00 & & & $47 \cdot 2$ \\
\hline & II & & $\mathbf{I}$ & 22.4 & +6 & 24.71 & + & 2.10 & & 0.00 & & & 49.2 \\
\hline & 14 & & 25 & 58.0 & -18 & 14.33 & + & 2.80 & & 0.00 & & & 46.5 \\
\hline & 95 & & 2 & I. 3 & +5 & 43.50 & + & $5 \cdot 50$ & & 0.00 & & & 50.3 \\
\hline & 16 & & $\mathbf{I}$ & 55.2 & +5 & 43.18 & + & $5 \cdot 50$ & & C. 00 & & & 43.9 \\
\hline & I9 & & 3 & 7.2 & +4 & 34.75 & + & $5 \cdot 50$ & & 0.00 & & & 47.5 \\
\hline & 26 & & $I_{4}$ & 1. 4 & -6 & 16.94 & + & $5 \cdot 50$ & & 0.00 & & & 50.0 \\
\hline & 32 & & 22 & 16.1 & -14 & 31.79 & + & 2.60 & & 0.00 & & & 46.9 \\
\hline & 33 & & 17 & $34 \cdot 7$ & -9 & 52.85 & + & 2.60 & & 0.00 & & & $44 \cdot 5$ \\
\hline & 34 & & 17 & $47 \cdot I$ & - IO & 2.33 & + & 2.60 & & 0.00 & & & $47 \cdot 4$ \\
\hline & 36 & & 3 & 56.8 & +3 & 43.46 & + & $4 \cdot 10$ & & 0.00 & & & $44 \cdot 4$ \\
\hline & 37 & & 10 & $9 \cdot 7$ & -2 & 24.21 & + & $4 \cdot 10$ & & 0.00 & & & 49.6 \\
\hline & 38 & & 7 & I. 4 & +0 & 40.71 & + & 6.30 & & 0.00 & & & 48.4 \\
\hline & 39 & & 14 & $29 \cdot 3$ & -6 & 48.70 & + & 2.80 & & 0.00 & & & $43 \cdot 4$ \\
\hline & $4 I$ & & 4 & 17.0 & +3 & 28.55 & + & 2.80 & & 0.00 & & & 48.4 \\
\hline & 42 & & 7 & $5^{8 . I}$ & -0 & 13.36 & + & o.10 & & 0.00 & & & $44 \cdot 9$ \\
\hline & 43 & & 5 & $5 \cdot 3$ & +2 & 39.75 & + & 0.10 & & 0.00 & & & 45.2 \\
\hline & 44 & & I4 & I I . 8 & -6 & 29.74 & + & $3 \cdot 30$ & & 0.00 & & & 45.4 \\
\hline & 44 & & I 5 & 12.2 & -7 & 29.35 & + & $5 \cdot 5^{\circ}$ & + & 0.10 & & & $48 \cdot 3$ \\
\hline & 45 & & 18 & 30.2 & -10 & 51.90 & $f$ & $5 \cdot 50$ & & 0.00 & & & 43.8 \\
\hline & 46 & & 13 & I0. 4 & -5 & 30.07 & + & $5 \cdot 30$ & & 0.00 & & & 45.6 \\
\hline & 48 & & I3 & $34 \cdot 7$ & -5 & $5 \mathrm{I} \cdot 48$ & + & $5 \cdot 30$ & & 0.00 & & & 48.5 \\
\hline & $4 \hat{9}$ & & 13 & $7 \cdot 4$ & -5 & 25.10 & + & $5 \cdot 30$ & & 0.00 & & & 47.6 \\
\hline
\end{tabular}


(s5.) Reduction of the Latitune-Observatrons-Centinued.

Computations for Latitude of Cheyenne, Wyoming Territory.

\begin{tabular}{|c|c|c|c|c|c|c|c|c|c|c|c|c|}
\hline \multirow{2}{*}{ Date. } & \multirow{2}{*}{$\begin{array}{l}\text { Number } \\
\text { of pair. }\end{array}$} & \multirow{2}{*}{\multicolumn{3}{|c|}{$\begin{array}{l}\text { Haif-sum of dec- } \\
\text { linsations. }\end{array}$}} & \multicolumn{6}{|c|}{ Cozrections. } & \multirow{2}{*}{\multicolumn{2}{|c|}{ Latifude. }} \\
\hline & & & & & \multicolumn{3}{|c|}{ Mic. ard ref. } & \multicolumn{2}{|c|}{ Level. } & Meridian. & & \\
\hline \multirow{9}{*}{$\begin{array}{l}\text { 1872. } \\
\text { Oct. } 9\end{array}$} & & o & , & $"$ & & , & $"$ & & $"$ & $"$ & $\circ \quad$, & " \\
\hline & $5 \mathrm{I}$ & $4 \mathrm{I}$ & 8 & 38.5 & - & o & 55.29 & + & 4.67 & 0.00 & 417 & 47.9 \\
\hline & 52 & & 12 & $7 \cdot 3$ & - & 4 & 26.82 & + & 7.60 & 0.00 & & 48.1 \\
\hline & 54 & & 4 & 13.4 & + & 3 & 31.03 & + & 2.75 & 0.00 & & 47.2 \\
\hline & 55 & & 14 & $59 \cdot 5$ & - & 7 & $\mathrm{I}_{5} \cdot 7 \mathrm{I}$ & + & I. 80 & 0.00 & & 45.6 \\
\hline & 56 & & & 7.8 & - & 9 & 24.88 & + & 2.79 & 0.00 & & 44.7 \\
\hline & 57 & & 13 & 17.4 & & 5 & 34.89 & + & 1.00 & 0.00 & & 43.5 \\
\hline & 60 & & 20 & 10. $\mathrm{x}$ & - & 12 & $24.2 \mathrm{I}$ & + & 0.70 & 0.00 & & 46.6 \\
\hline & 59 & & I3 & 18.0 & & 5 & 32.40 & $\rightarrow$ & 0.70 & 0.00 & & 46.3 \\
\hline \multirow[t]{34}{*}{ Oct. $\quad$ Io } & 1 & 40 & 55 & 42.9 & + & I 2 & I.99 & & 3.30 & 0.00 & $\begin{array}{ll}417 & 7\end{array}$ & 48.2 \\
\hline & 4 & $4 I$ & 5 & 33.2 & + & 2 & 10.54 & & 2.47 & 0.00 & & 45.2 \\
\hline & 5 & 40 & 56 & $49 \cdot 9$ & & 10 & $52 \cdot 37$ & & $4 \cdot$ ro & 0.00 & & 46.4 \\
\hline & 6 & $4 \mathrm{I}$ & 8 & 22.2 & - & o & 39.47 & + & 4. so & 0.00 & & 46.8 \\
\hline & 7 & & 9 & 26.4 & - & $\mathbf{I}$ & 44.80 & + & 4.10 & 0.00 & & 45.7 \\
\hline & 8 & & 15 & 12.8 & - & 7 & 28.65 & + & 2.60 & 0.00 & & 46.8 \\
\hline & 9 & & 18 & 28.8 & - & 10 & $4^{6.93}$ & $\rightarrow$ & 2.60 & 0.00 & & 44.5 \\
\hline & I I & & I & 22.5 & + & 6 & 19.80 & & 3.40 & 0.00 & & $45 \cdot 7$ \\
\hline & 12 & & 9 & 13.2 & - & I & 28.67 & + & 2.86 & 0.00 & & $47 \cdot 4$ \\
\hline & I3 & 40 & 57 & $5^{1} \cdot 5$ & + & 9 & 51.92 & & $2 . y 0$ & 0.00 & & 46.3 \\
\hline & 15 & $4 I$ & 2 & I. 4 & + & 5 & 41.22 & + & 3.40 & 0.00 & & 46.0 \\
\hline & 16 & & I & $57 \cdot 3$ & & 5 & 45.94 & & 3.40 & 0.00 & & 46.6 \\
\hline & 17 & & 3 & $\bar{I} \cdot \bar{B}$ & + & 4 & $35 \cdot 37$ & & 3.40 & 0.00 & & 45.1 \\
\hline & 25 & & 16 & 29.6 & - & 8 & 49.60 & + & 5.10 & 0.00 & & $45 . I$ \\
\hline & 26 & & 14 & 1.5 & - & 6 & 18.55 & + & 4.40 & 0.00 & & $47 \cdot 3$ \\
\hline & 33 & & 17 & $47 . \dot{2}$ & - & 10 & 4.19 & + & $5 \cdot 50$ & 0.00 & & 48.5 \\
\hline & 34 & & 17 & 33.8 & - & 9 & $54 \cdot 40$ & + & 5.50 & 0.00 & & 45.9 \\
\hline & 36 & & 3 & 57.5 & + & 3 & $47.5 \mathrm{I}$ & + & I. 80 & 0.00 & & 46.8 \\
\hline & 37 & & 10 & 9.8 & - & 2 & 25.14 & + & x. 78 & 0.00 & & 46.4 \\
\hline & $3^{8}$ & & 7 & I. 6 & + & o & 39.16 & + & $4 \cdot 40$ & 0.00 & & 45.2 \\
\hline & 39 & & $x_{4}$ & 29.5 & - & 6 & +5.53 & + & I. 50 & $0 . \infty 0$ & & 45.5 \\
\hline & 40 & & 4 & 17.2 & + & 3 & 25.13 & & 4.90 & 0.00 & & 47.2 \\
\hline & 43 & & $\mathrm{I}_{4}$ & 12.0 & - & 6 & 30.12 & & $5 \cdot 30$ & 0.00 & & 47.2 \\
\hline & 44 & & 15 & 12.4 & - & 7 & 30.50 & + & 4.30 & $0 . \infty 0$ & & 46.2 \\
\hline & 45 & & 18 & 30.4 & - & ro & $40 \cdot ? ?$ & + & $4 \cdot 30$ & 0.00 & & $45 \cdot 3$ \\
\hline & 46 & & 13 & I0. 7 & - & 5 & 26.34 & & 3.20 & 0.00 & & 47.6 \\
\hline & 48 & & 13 & 34.9 & - & 5 & 50.40 & + & 3.20 & 0.00 & & 47.7 \\
\hline & 49 & & I3 & 7.6 & - & 5 & 24.01 & & 3.20 & 0.00 & & 46.8 \\
\hline & 50 & & 8 & $3 \hat{5} .7$ & - & o & $53.6 \mathrm{I}$ & + & I. $3^{8}$ & 0.00 & & 46.5 \\
\hline & 52 & i & 12 & $7 \cdot 5$ & - & 4 & 25.22 & $\div$ & 4.20 & 0.00 & & 43.5 \\
\hline & 53 & & 15 & $5 \cdot 5$ & - & 7 & 24.45 & + & 5.94 & 0.00 & & 47.0 \\
\hline & 54 & : & 4 & 13.4 & + & 3 & $2 S .56$ & + & 2.75 & 0.00 & & 45.0 \\
\hline & 55 & i & $I_{4}$ & 59.7 & - & 7 & 16.02 & + & 2.33 & 0.00 & & 46.0 \\
\hline & 56 & & 17 & 8.0 & - & 9 & 23.95 & + & $2.3^{\circ}$ & c.co & & 46.4 \\
\hline
\end{tabular}


(15.) Reduction of the Latitude-Observations-Contimued

Computations for Latitude of Cheigenne, Wyoming Territory.

\begin{tabular}{|c|c|c|c|c|c|c|c|c|c|c|c|}
\hline \multirow{2}{*}{ Date. } & \multirow{2}{*}{$\begin{array}{l}\text { Number } \\
\text { of pair. }\end{array}$} & \multirow{2}{*}{\multicolumn{3}{|c|}{$\begin{array}{l}\text { Half-sum of dec̀- } \\
\text { linations. }\end{array}$}} & \multicolumn{5}{|c|}{ Corrections. } & \multirow{2}{*}{\multicolumn{2}{|c|}{ Latitude. }} \\
\hline & & & & & Mic. a & and ref. & & evel. & Meridian. & & \\
\hline \multirow{5}{*}{$\begin{array}{c}187.2 . \\
\text { Oct. 1o }\end{array}$} & & $\circ$ & ' & 7 & ' & $"$ & & " & $"$ & - 1 & $"$ \\
\hline & 57 & $4 \mathrm{I}$ & 13 & 17.6 & -5 & $33 \cdot 33$ & + & $\mathrm{I} .65$ & $0 . \infty 0$ & $4 I 7$ & 45.9 \\
\hline & $5^{8}$ & 40 & 57 & $I I, 5$ & + ro & 33.88 & + & 1.30 & 0.00 & & 46.7 \\
\hline & 60 & $4 I$ & 20 & 10.5 & -12 & $24 \cdot 74$ & + & 0.60 & 0.00 & & 46.4 \\
\hline & 59 & & 13 & 18.2 & -5 & $31 \cdot 38$ & + & 0.55 & $0 . \infty 0$ & & 47.4 \\
\hline \multirow[t]{38}{*}{ Oct. II } & I & $4 I$ & 4 & 12. I & +3 & 29.01 & $\dot{+}$ & $5 \cdot 50$ & 0.00 & 417 & 46.6 \\
\hline & 2 & 40 & 55 & 42.9 & +21 & $58.4 \mathrm{I}$ & + & $5 \cdot 50$ & 0.00 & & 46.8 \\
\hline & 3 & $4 I$ & 5 & 0.0 & +2 & $4 I .7 I$ & + & 5.08 & 0.00 & & 46.8 \\
\hline & 5 & 40 & 56 & 50.0 & $+1 I$ & $\mathrm{I} \cdot 38$ & - & 2.75 & 0.00 & & 48.6 \\
\hline & 6 & $4 I$ & 8 & 22.3 & -0 & 32.54 & - & 2.70 & 0.00 & & 47.1 \\
\hline & 7 & & 9 & 26.4 & -1 & $38.7 x$ & - & 2.33 & 0.00 & & $45 \cdot 4$ \\
\hline & 8 & & 15 & 12.9 & -7 & 23.82 & - & $3 \cdot 74$ & 0.00 & & $45 \cdot 3$ \\
\hline & 9 & & is & 28.9 & -10 & 38.54 & & 3.20 & 0.00 & & 47.2 \\
\hline & ro & 40 & 57 & 30.0 & +10 & 22.34 & & 4.40 & 0.00 & & 48.0 \\
\hline & II & $4 I$ & I & 22.5 & +6 & 25.08 & & I. 65 & 0.00 & & 45.9 \\
\hline & 12 & & 9 & 13.3 & $-I$ & 23.76 & - & 2.90 & $0 . \infty 0$ & & 46.6 \\
\hline & 13 & 40 & 57 & $5 \mathrm{r} .6$ & +9 & 57.30 & & 2.88 & 0.00 & & 46.0 \\
\hline & 14 & $4 \pi$ & 25 & $5^{8.2}$ & -18 & II.06 & & I. 23 & 0.00 & & 45.9 \\
\hline & I5 & & 2 & 1.5 & +5 & 50.74 & & 3.40 & 0.00 & & 48.8 \\
\hline & 16 & & I & 57.4 & +5 & 52.14 & & 3.40 & 0.00 & & 46. I \\
\hline & 18 & & 3 & II. .4 & +4 & 36.26 & & 3.40 & 0.00 & & $44 \cdot 3$ \\
\hline & 24 & & 3 & 6.8 & +4 & 42.39 & & 2.40 & 0.00 & & 46.8 \\
\hline & 26 & & 14 & ฯ.7 & -6 & 9.42 & & 3.80 & 0.00 & & 48.5 \\
\hline & $3 \mathrm{I}$ & & 22 & 37.7 & $-I_{4}$ & 48.05 & & 3.98 & $0 . \infty 0$ & & $45 \cdot 7$ \\
\hline & 36 & & 10 & 10.0 & -2 & 21.66 & & 2.60 & 0.00 & & $45 \cdot 7$ \\
\hline & 38 & & 7 & I. 7 & +0 & 46.09 & & 2.75 & 0.00 & & 45.0 \\
\hline & $\$ 9$ & & 14 & 29.8 & -6 & 37.42 & & 4.80 & 0.00 & & 47.6 \\
\hline & 40 & & 4 & 17.4 & +3 & $33.6 \mathrm{r}$ & & $3 \cdot 70$ & 0.00 & & 47.5 \\
\hline & $4^{I}$ & & 7 & 58.6 & -0 & IIIIO & & $2 . \infty 0$ & 0.00 & & 45.5 \\
\hline & 42 & & 5 & 5.8 & +2 & 42.02 & - & 2.00 & 0.00 & & 45.8 \\
\hline & 43 & & 14 & 12.3 & -6 & $23 \cdot 37$ & & 4.40 & 0.00 & & 44.5 \\
\hline & 44 & & I5 & 12.7 & -7 & 19.78 & & $5 \cdot 50$ & 0.00 & & $47 \cdot 4$ \\
\hline & 45 & & 98 & 30.7 & - ro & 38.69 & & 5.50 & 0.00 & & 46.5 \\
\hline & 46 & & 13 & 10.9 & -5 & I9. I9 & & 4.67 & 0.00 & & 47.0 \\
\hline & 48 & & 13 & 35.2 & -5 & 43.00 & & 4.60 & 0.00 & & 47.6 \\
\hline & 49 & & I3 & 7.9 & -5 & 16.70 & & 4.60 & 0.00 & & 46.6 \\
\hline & $5 I$ & & 9 & 39.0 & -0 & 50.97 & & 2.00 & 0.00 & & 46.0 \\
\hline & 52 & & 12 & 3.7 & -4 & 20.67 & - & 2.33 & 0.00 & & 44.7 \\
\hline & 53 & & 15 & 5.8 & -7 & 17.20 & & 2.33 & 0.00 & & 46.3 \\
\hline & 54 & & 4 & 13.4 & +3 & 38.49 & & 5.08 & 0.00 & & 46.8 \\
\hline & 55 & & I5 & 0.0 & -7 & 6.42 & & 3.85 & 0.00 & & $49 \cdot 7$ \\
\hline & 56 & & 17 & 8.2 & -9 & 14.47 & & 3.80 & 0.00 & . & 49.9 \\
\hline & 57 & & 13 & 17.8 & -5 & 23.24 & & 6.60 & $0 . \infty$ & & 48.0 \\
\hline
\end{tabular}




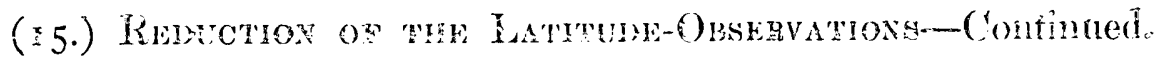

Computations for Latitude of Cheyenne, Wyoming Tervitory.

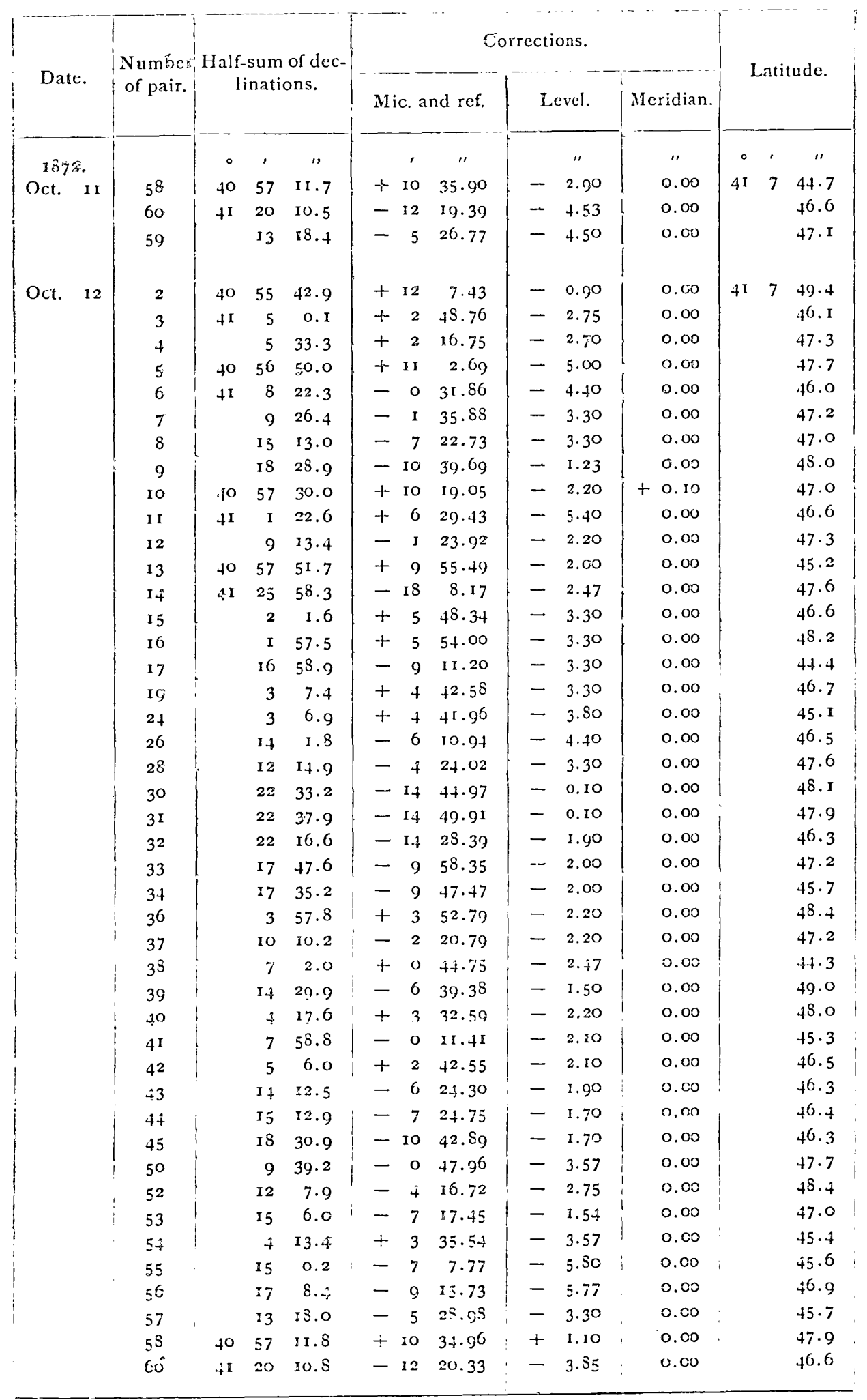


(15.) Observations for Latitude, Cheyenne, Wyoming 'Territory.

\section{Recapitulation.}

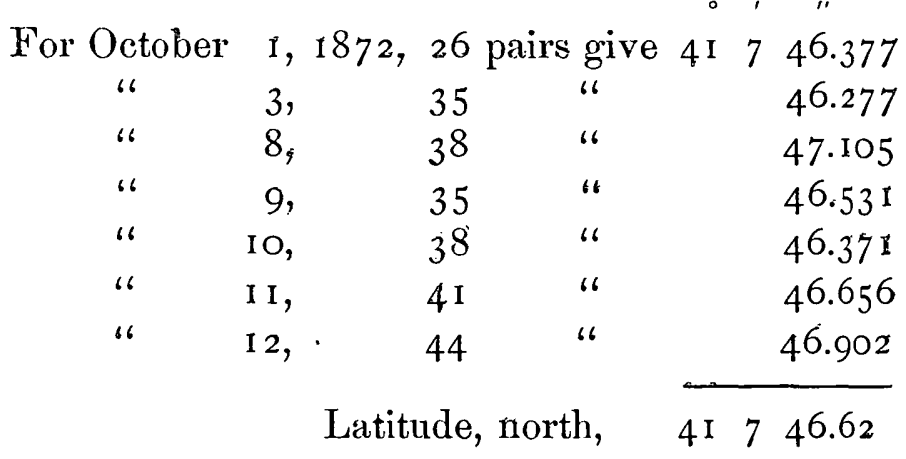

Giving the first series half-weight on account of the smaller number of the observations and the less favorable condition of that night's work, the resulting latitude, and the one adopted for this station, is, $4 \mathrm{I}^{\circ} 7^{\prime} 46^{\prime \prime} .62$, with a probable error of $\pm 0^{\prime \prime} .08$. The latitudes were originally computed by Professor William A. Rogers, of Cambridge, Mass., and revised by Dr. F. Kampf.

\section{(i6.) Resulting Astronomical Co-ordinates.}

Taking the longitude of the Salt Lake observatory to be $2^{\mathrm{ht}} 19^{\mathrm{m}} 22^{\mathrm{s}} \cdot 74$ west of Washington by determination of the United States Coast Survey, and Washington to be $5^{\mathrm{h}} 8^{\mathrm{m}}$ I $2^{\mathrm{s}}$. I 2 west of Greenwich according to the report of Rear-Admiral B. F. Sands, Superintendent of the United States Naval Observatory, October 6, I87 I, Cheyenne is in longitude west from Washington, in time, $\mathrm{I}^{\mathrm{l}} 5 \mathrm{I}^{\mathrm{m}} 3^{8} \cdot 30$; in are, $27^{\circ} 45^{\prime} 49^{\prime \prime} .50$; in longitude west from Greenwich, in time, $6^{\mathrm{h}} 59^{\mathrm{m}} 15^{\mathrm{s}} \cdot 4^{2}$; in are, $104^{\circ} 48^{\prime} 5 \mathrm{I}^{\prime \prime} .30$; in latitude, north, $4 \mathrm{I}^{\circ} 7^{\prime} 46^{\prime \prime} .62 \pm \mathrm{O}^{\prime \prime} .08$.

This final result for longitude is subject, as already stated, to a correction for the personal equation of the observers. It is possible, also, that the longitude of Salt Lake may be changed when the observations made last October at Detroit and Ogden by the United States Lake Survey and your expedition respectively are computed. In such an event, of course the longitude of Cheyenne will be correspondingly affected.

Respectfully, yours,

Lieut. Geo. M. Wheeler,

JOHN H. CLARK.

Corps of Engineers, in charge. 



\section{R E P O R'T}

ON

\section{ASTRONOMICAL OPERATIONS,}

CONDUCTED DURING

\section{THE FIELD-SEASON OF 1873,}

$\mathbf{A T}$

THE MAIN OR PRIMARY FIELD-STATION, COLORADO SPRINGS, COLORADO TERRITORY,

AND

DEDUCTION OF RESULTS.

BY

Dr. F. KAMPF,

CIVILIAN ASTRONOMICAL ASSISTANT. 



\section{U. S. Engineer Office, Geographical and Geological Explorations and Surveys West of iooth Meridian, Washington, D. C., January I, I 874 .}

SIR: There is presented herewith a report upon the astronomical observations taken by myself, and the party under my charge, at Colorado Springs, Colorado Territory, during the field-season of 1873 .

$$
\begin{aligned}
& \text { Geographical Position of Station. } \\
& \text { Longitude }=104^{\circ} 49^{\prime} \text { I } 5^{\prime \prime} \text {. IO. } \\
& \text { Latitude }=38^{\circ} 49^{\prime} 4 \mathrm{I}^{\prime \prime} .67 .
\end{aligned}
$$

Colorado Springs is a town in El Paso County, Colorado Territory. It has been built up within five years, and has nearly fifteen hundred inhabitants, and the place promises to become one of considerable importance. During the summer-months the hotels (of which there are quite a large number) are filled with invalids, who flock here on account of the beautiful scenery and the salubrity of the climate. The track of the Denver and Rio Grande Railway passes around the town at a distance from the town-limits of about four thousand feet.

The astronomical point is situated between the town and the railroad, about six hundred and fifty feet distant from the latter, on a slight eminence near the freightdepot of the Denver and Rio Grande Railroad. The observations were conducted on a pier built of bricks, which was replaced two months later by a solid sandstone monument, furnished by Mr. S. G. Ward, of Pueblo.

\section{Physical-Geography Details.}

This part of Colorado is not well watered, but the land where irrigated yields almost in every instance splendid harvests.

From the astronomical point there is a clear outlook to the north, south, and east. Looking west, prominent peaks and foot-hills of the Rocky Mountain range are seen running north and south ; Pike's Peak, immediately west, being the highest, and Cheyenne Mountain the highest in the south-southwest. At the foot of Cheyenne Mountain there is a creek, the waters of which are brought, by means of ditches, to Colorado Springs. From the station the plains rise a little to the east, at the horizon say one hundred and fifty feet. In the southeast there is a hill about four hundred feet high, called Washington Mountain.

Colorado Springs is laid out regularly, the streets running east and west and north and south; the greatest extension is from north to south.

Generally speaking, it is inadvisable to have the astronomical station near the railroad-track; but in this case the trains ran only during the day, and the observations were never affected by the vibrations of the ground. 


\section{Mrteorological Conitions.}

The meteorological observations made at the station show great changes in temperature during the day. I have been told by several old residents that they never experienced a summer similar to that of 1873 . The rainy period of the summer

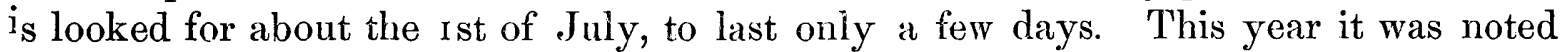
that from July 28th to August 9th there was no day without rain, accompanied by thunder and lightining. The prevailing winds were from the northeast or southeast, commencing at Io or I I o'clock a. m., and increasing in force until 2 or 3 o'clock p. m. Then clouds came up from the southwest or west, bringing much rain, thunder, and lightning. It was generally clear again by i 2 p. m.; but I found the air so very undulating, and the stars on that account so faint, that I was sometimes obliged to suspend the observations. It is probable that the temperature of the higher regions of the air was affected by the vicinity of the mountains, and after a rain changed very rapidly, while the lower strata remained under the same conditions.

The following table shows the general direction of the wind at 7 a. m., 2 p. m., and 9 p. m., giving the mean or prevailing direction of the wind for three hours before and three hours after the given time; also the estimated force of wind for the same time. The last column gives the general appearance of the sky, and needs no further explanation. It shows under what particularly unfavorable circumstances the observations were made:

\begin{tabular}{|c|c|c|c|c|c|c|c|c|}
\hline \multicolumn{2}{|c|}{ Date. } & \multicolumn{3}{|c|}{ Direction of wind. } & \multicolumn{3}{|c|}{ Estimated force. } & Remarks. \\
\hline \multicolumn{9}{|l|}{ I873. } \\
\hline July & 28 & . & S. & NE. & . & 2 & $\mathbf{I}$ & Cloudy during the day; clear after 12 p. m. \\
\hline & 29 & 0 & E. & SE. & o & 2 & 2 & Clear; heavy dew in the night. \\
\hline & 30 & N. & sw. & NW. & $\mathbf{I}$ & 3 & 2 & Heavy wind and rain in the afternoon. \\
\hline & $3 \mathrm{I}$ & $\mathrm{N}$ & $\mathrm{SE}$ & $\mathrm{N}$. & $\mathbf{r}$ & r & I & Rain from the west at 3 p. $m$. \\
\hline \multirow{10}{*}{ August } & $\mathbf{I}$ & N. & NE. & NW. & 2 & 4 & 3 & Heavy shoiver in the aftcrnoon; storm from northeast at 9 p. $m$. \\
\hline & 2 & 0 & NE. & S. & o & 5 & 3 & Heavy rain, with thunder and lightning, at 3 p. m. \\
\hline & 3 & S. & NE. & $\mathrm{SE}$. & 2 & 3 & 2 & Cloudy ail day. \\
\hline & 4 & 0 & SE. & $\mathrm{N}$. & $\circ$ & 3 & I & Cloudy all day; rain in the afternoon. \\
\hline & 5 & 0 & SE. & NW. & ○ & 2 & I & Ciear in the morning, clouchy in the dîtintavoñ. \\
\hline & 6 & NW. & NW. & S. & I & 2 & I & Cloudy all day; rain in the afternoon. \\
\hline & 7 & 0 & $S E$. & Nw. & $c$ & 2 & I & Cloudy all day; storming from the northwest at $12 \mathrm{~m}$. \\
\hline & $s$ & N. & N. & $\mathrm{SE}$. & $\underline{\mathbf{I}}$ & 4 & 4 & Cinudy alt day. \\
\hline & 9 & NW. & SE. & $\mathrm{SE}$. & $\mathbf{I}$ & 2 & $\mathbf{I}$ & Cloudy all day; rain at 9.13 p.m. \\
\hline & Io & NE. & . & . & $\mathbf{I}$ & - & . & Clear. \\
\hline
\end{tabular}

\section{Description of Observatory at Colorado Springs, Colorado Territory.}

As soon as the monument was built, a solid framework, 8 by ro feet, was constructed, and a large wall-tent put orer it. There was an opening in the tent for the meridian-line; this was closed, when necessary, by a fly. During heavy winds the 
tent was in danger of being blown away, and it was found necessary to nail the fly to the framework, while the rent itself was fastened to the stakes hy strong iron wires. The entrance to the tent was from the west side, and was closeri hy ropes. In the northwest corner of the observatory a large box was used for a table. (On it the switchboard and galvanic battery were placed; the chronometer heing also placed there during the observations. The connection from the switch-hoard to the Western Union Telegraph office was made by a line 600 feet in length, supported by the framework of the tent and one telegraph-post 30 feet in height. A ground-wire was used after switching in the Westem Union office to complete the circuit. In the northeast comer of the tent the chronograph was placed upon a solid and insulated franework. Wires for the connection of the chronometer and breaking-key were fastened to the tent-fiame. The levels were also set on an insulated post in the southeast comer of the tent. For chairs I used two small boxes, one on the north and the other on the south side of the monument. In arranging and constructing the observing-tent I was assisted by C. I). Gerney and Privates J. Meier and J. Clancy, Battalion of Engineers. They also took the meteorological observations. Mr. G. 'T. Ellison, at that time in charge of the Westem Union office, kindly assisted in sending the telegraphic signals.

\section{Description of Instruments Useio.}

Observations were made by means of a combined transit-instrument number: 28 , made by $W$ ürdemann. Its focal length is three feet; radius of aperture, $2 \underset{4}{3}$ inches; diameter of pivots, $1 \frac{1}{4}$ inches. The diagonal eye-piece used had a magnifying-power of 40 diameters. This instrument was provided with two finding-circles, $3 \frac{1}{2}$ inches in diameter, graduated to every twenty minutes, and reading to single minutes by means of the vernier. Another circle was affixed to the upper part of the tube, divided also also to twenty minutes, and having in the center a level used in latitude-observations for determining the change in the inclination of the horizontal revolving-base. Seven wires were placed in the focus for time-observations, besides one horizontal wire for latitude-observations. The equatorial intervals of the wires from mean of wires, clamp west, upper culmination, were :

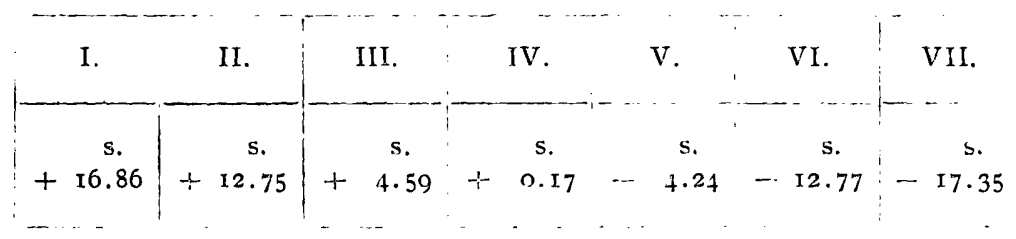

One revolution of the micrometer-screw moved the horizontal-wire $62^{\prime \prime}$. I 2 ; the the value of one division of the striding-level, which was used at every station, was $\mathrm{O}^{\prime \prime} .75$; the value of one division of the zenith-telescope level was $\mathrm{I}^{\prime \prime} .10$. The chronograph used was similar to that used at the United States Naval Observatory invented by Professor William Harkness; the barrel being 8 inches in diameter and 24 inches long, and makes one revolution a minute. The chronograph worked rery well when it was cleaned before commencing operations at a new station. It had but a single pen, which recorded clock-signals and those made by the observer. 
For time-observations and exchange, sidereal chronometer No. I49I, Negrts, was alway used. The galvanic connections were made by means of a switch-board, the comnections of which are given in the following diagram:

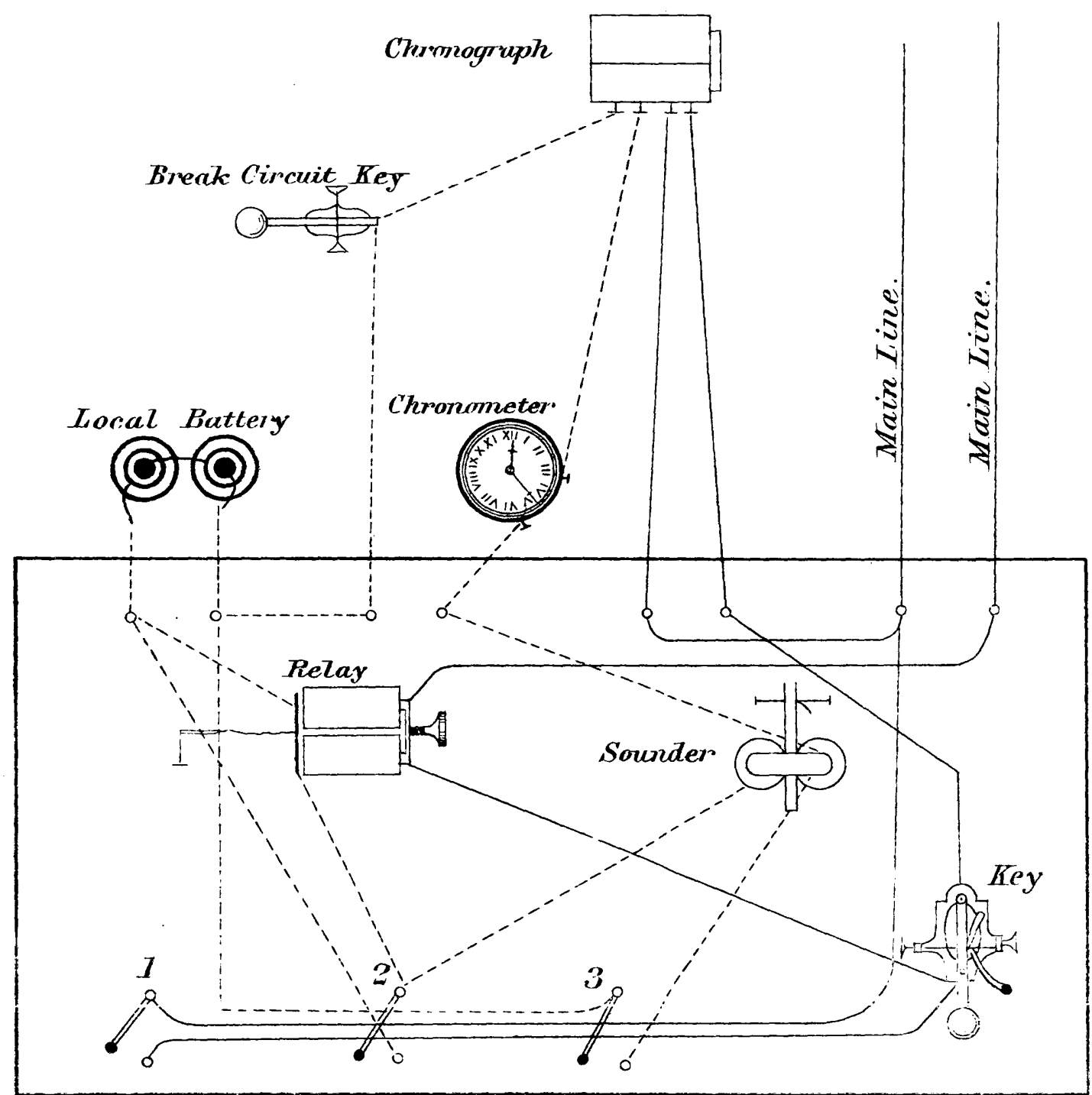

SwItch 1.-Closed when receiring fiom connected station; Nos. 2 and 3 open. Smitch 2.-Closed when sending to connected station; Yos. I and 3 open. SWITCH 3.-Local; throws sounder into local cireuit. 
Points with which Connections were Made, \&c.

Connection was made with Salt Lake City on the nights of July 29th and 3oth, and August 2d, 5th, and 6th. Observations for time were made at Colorado Springs on the nights of July 28th, 29th, 3oth, and 3ist, and August 2d, 4th, 5th, and 6th; at Salt Lake on the nights of July 28th, 29th, 3oth, and 3 Ist, and August Ist, 2d, 5 th, and 6th.

The reductions of time-observations for Colorado Springs were made in the field by the astronomer, and also those made at Salt Lake after returning from the field. $\mathrm{He}$ also made a new reading of the signals sent and received from both stations.

The telegraph-line between Colorado Springs and Salt Lake is $76_{3}$ miles long, and divided into four circuits. The signals are transmitted from one circuit to another by means of automatic repeaters: one placed at Denver, Colorado Territory; one at Cheyenne, Wyoming Territory; and one at Corinne, Utah: the length of the line being from Colorado Springs to Denver, 75 miles; from Denver to Cheyenne, Io6 miles; from Cheyenne to Corinne, 537 miles; and from Corinne to Salt Lake, 45 miles; using at every station sixty-five Grove cells.

The use of the wires was always freely tendered by the Western Union Telegraph Company, although in many cases they were needed at the same time for the transaction of the regular business of the company.

It sometimes occurs when two lines of wires are fixed to the same poles that, during heavy storms, the wires are brought in contact by oscillation, which was overcome in this case by connecting the two wires at an intermediate station, Denver, Colorado, upon the suggestion of Mr. Woodward, the superintendent at that point. 
Tabulation of Stars used for Determination of Time at Colorado Springs, Colorado, and Salt Lake City, Utah.

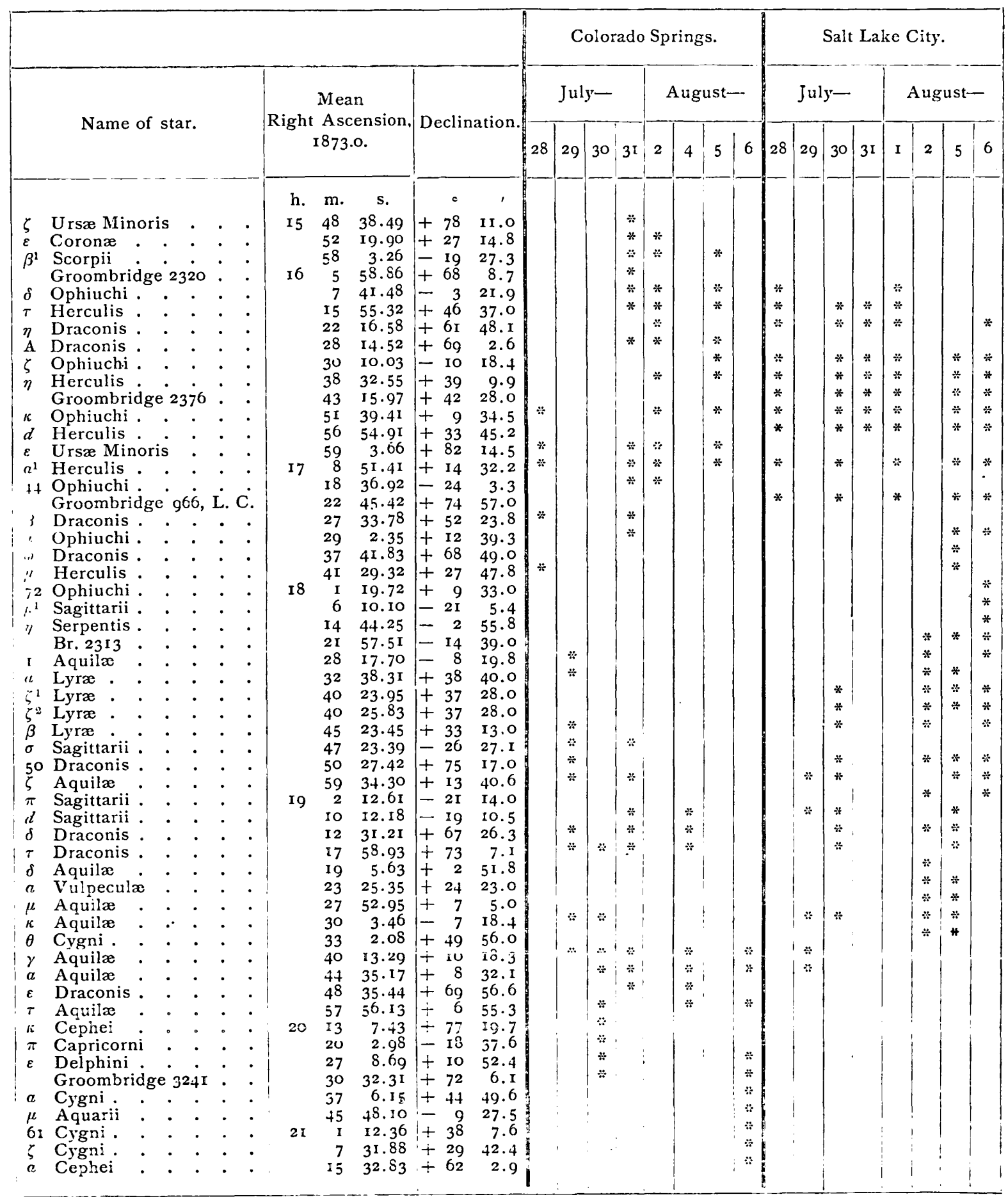


COLORADO SPRINGS, COLORADO TERRITORY, $\mathcal{F}_{u l y ~} 28,1873$.

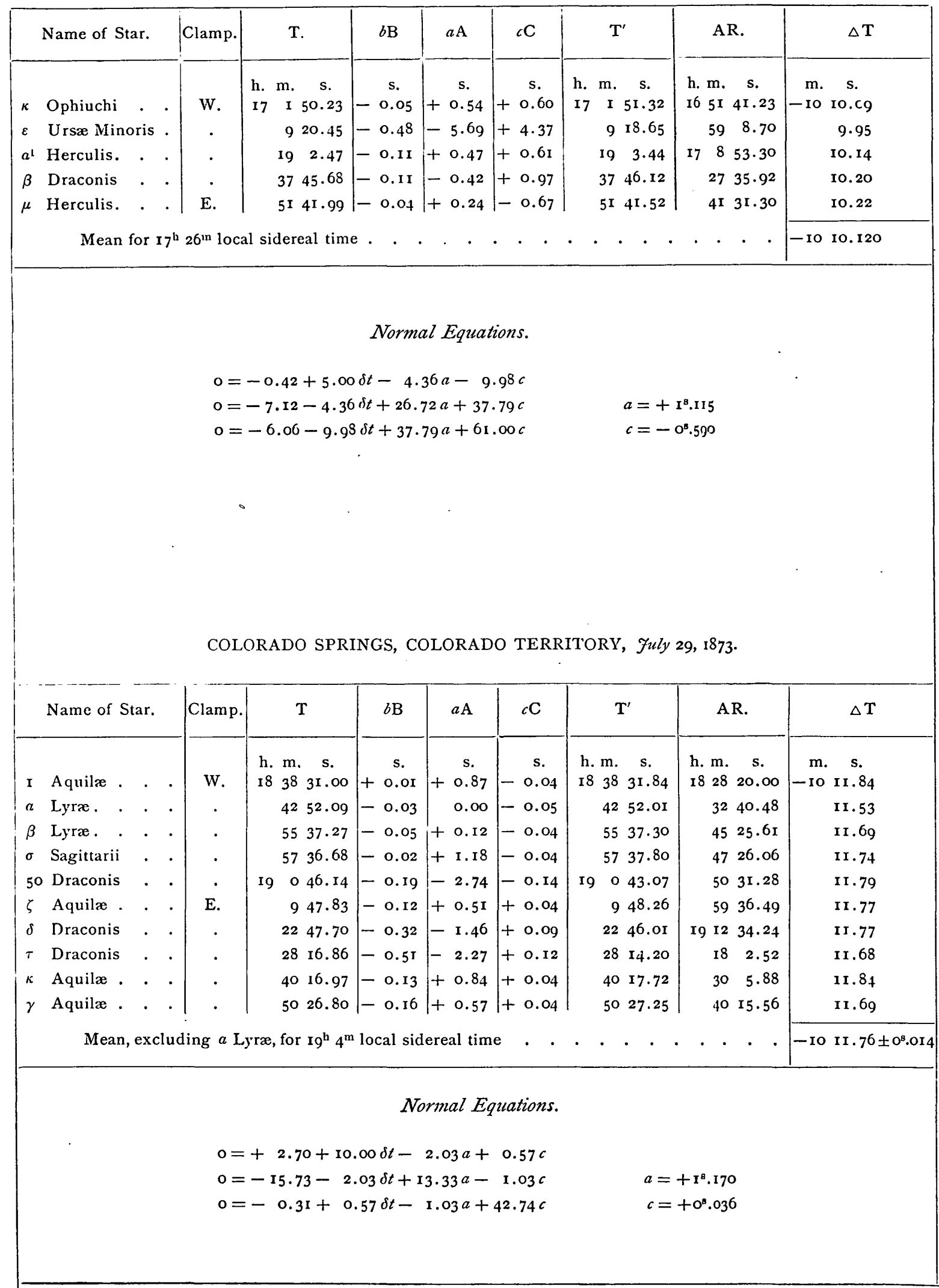


COLORADO SPRINGS, COLORADO TERRITORY, $\mathcal{f u l y}^{\prime} 3^{\circ}, \mathbf{1} 873$.

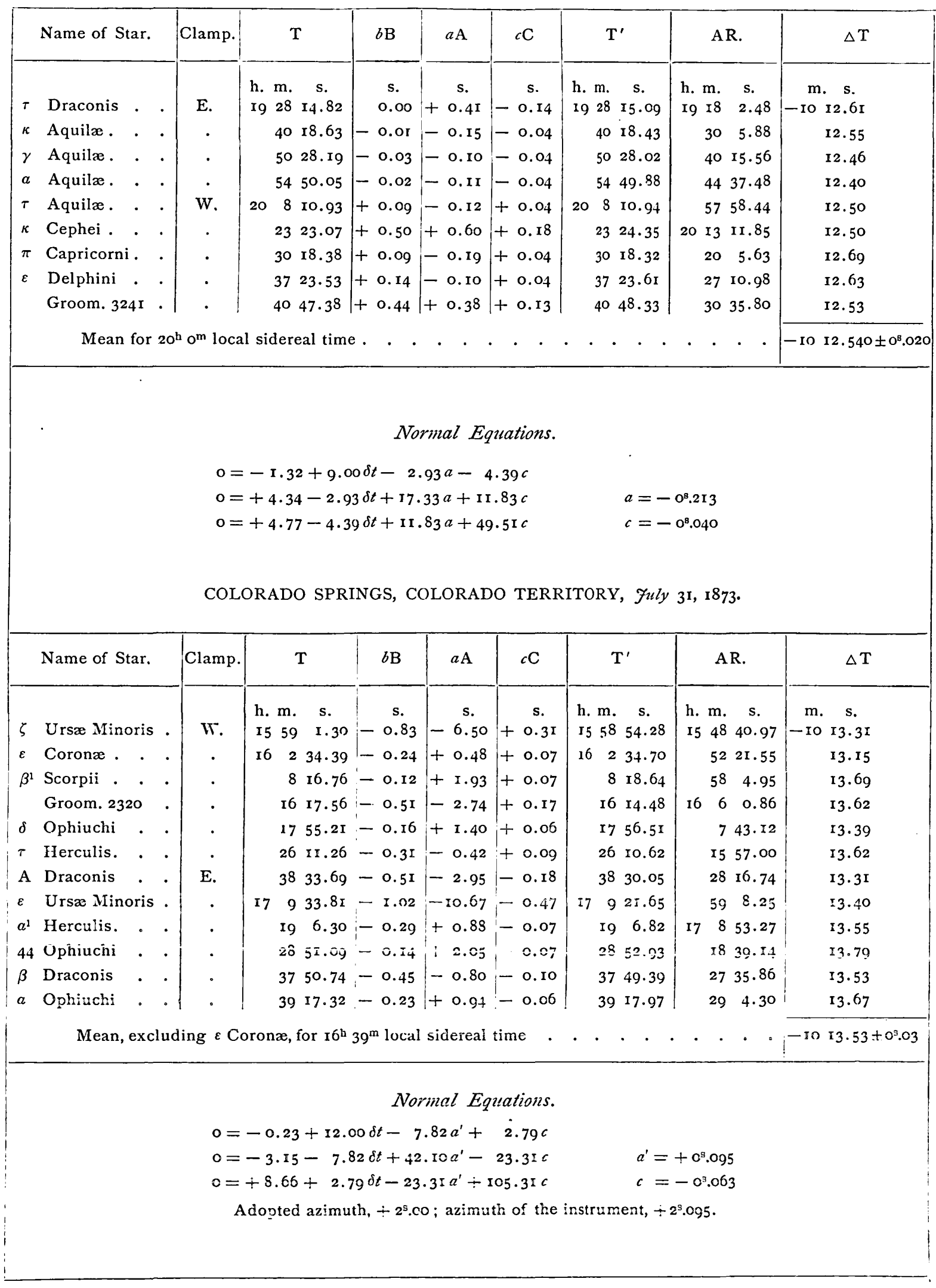


COLORADO SPRINGS, COLORADO TERRITORY, $\mathcal{F}_{i l y}$ 31, i873.

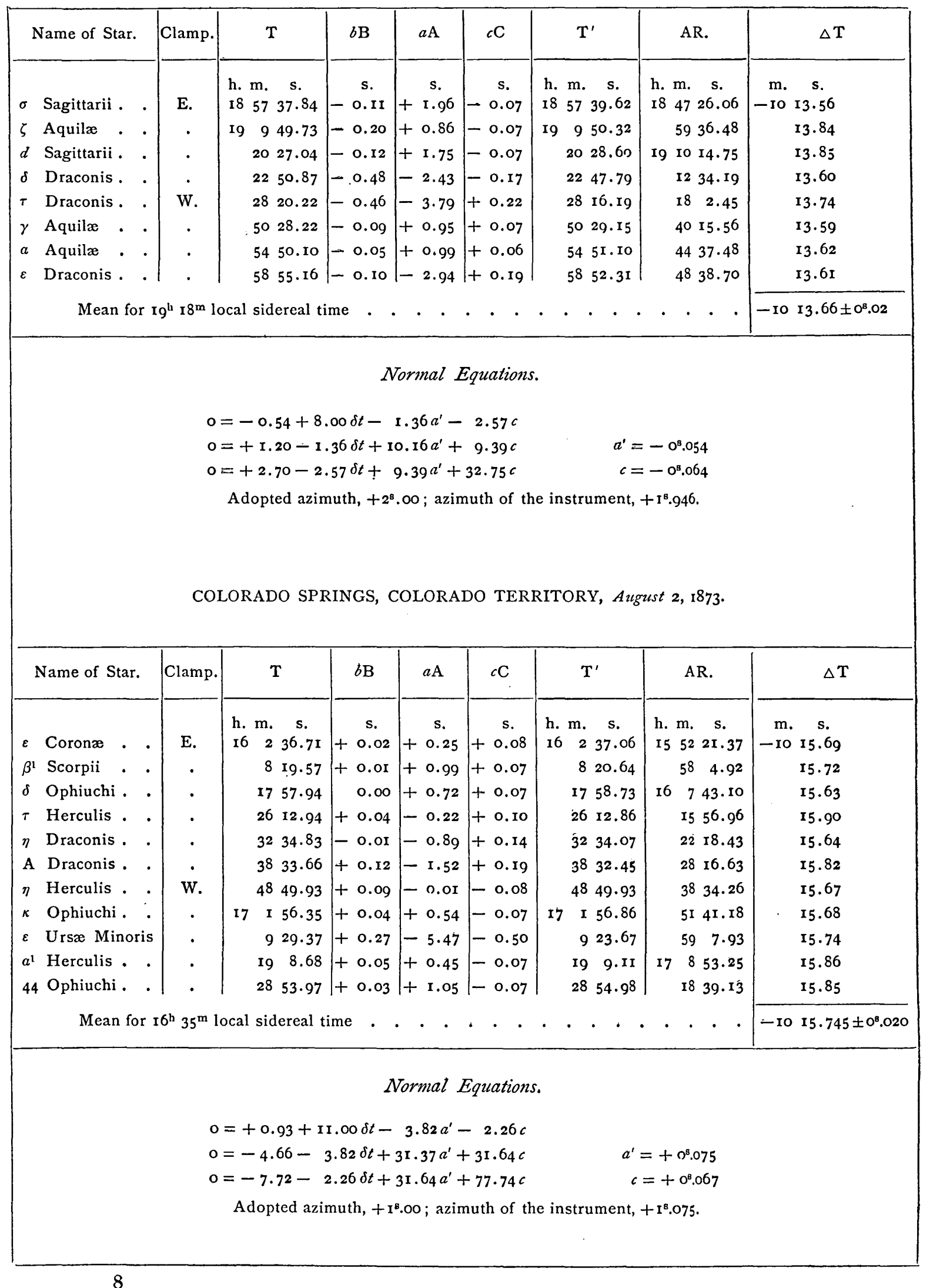


COLORADO SPRINGS, COLORADO .TERRITORY, August 4, 1873 .

\begin{tabular}{|c|c|c|c|c|c|c|c|c|c|}
\hline & Name of Star. & Clamp. & $\mathrm{T}$. & $b \mathrm{~B}$ & $a \mathrm{~A}$ & $c \mathrm{C}$ & $\mathrm{T}^{\prime}$ & AR. & $\Delta \mathrm{T}$ \\
\hline$d$ & Sagittarii . & E. & $\begin{array}{ccc}\text { h. m. } & \text { s. } \\
\text { I9 } & 20 & 31.64\end{array}$ & $\begin{array}{c}\text { s. } \\
-0.04\end{array}$ & $\begin{array}{c}\text { s. } \\
+\quad 1.27\end{array}$ & $\begin{array}{c}\mathrm{s} . \\
-0.04\end{array}$ & 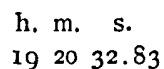 & $\begin{array}{l}\text { h. m. s. } \\
\text { 19 10 1 } 4.76\end{array}$ & $\begin{array}{c}\text { m. s. } \\
- \text { - } 10 \text { 18.07 }\end{array}$ \\
\hline$\delta$ & Draconis . & . & $22 \quad 54.29$ & -0.23 & -1.77 & -0.09 & 2252.20 & I2 34.10 & I8. I0 \\
\hline - & Draconis . & . & $28 \quad 23.53$ & -0.34 & -2.73 & -0.12 & $28 \quad 20.34$ & $28 \quad 2.33$ & 18.01 \\
\hline$\gamma$ & Aquilæ & W. & $50 \quad 32.95$ & -0.04 & +0.69 & +0.04 & $50 \quad 33.64$ & $40 \quad 15.57$ & I8.07 \\
\hline$a$ & Aquilæ & . & 5454.92 & -0.02 & +0.72 & +0.03 & 5455.65 & $44 \quad 37.49$ & I8. I6 \\
\hline$\varepsilon$ & Draconis . & 。 & $5^{8} \quad 58.68$ & 0.00 & -2.13 & +0.10 & $5^{8} \quad 56.65$ & $48 \quad 38.63$ & 18.02 \\
\hline$\tau$ & Aquilæ & . & 815.76 & $1+0.04$ & +0.76 & +0.04 & 20816.60 & 5758.46 & I8. 14 \\
\hline
\end{tabular}

Mean for $19^{\mathrm{h}} 44^{\mathrm{m}}$ local sidereal time . . . . . . . . . . . . . . . . $-10 \mathrm{18.081 \pm 08.014}$

\section{Normal Equations.}

$\begin{array}{ll}0=+0.16+7.00 \delta t-2.26 a^{\prime}+1.15 c & \\ 0=-0.93-2.26 \delta t+7.20 a^{\prime}-6.16 c & a^{\prime}=+0^{8} .110 \\ 0=+1.58+\mathrm{I} .15 \delta t-6.16 a^{\prime}+31.46 c & c=-0^{8} .034\end{array}$

Adopted azimuth, $+\mathrm{I}^{\mathrm{B}} \cdot 30$; azimuth of the instrument, $+\mathrm{I}^{\mathrm{B}} \cdot 4 \mathrm{IO}$ 。

COLORADO SPRINGS, COLORADO TERRITORY, August 5, 1873.

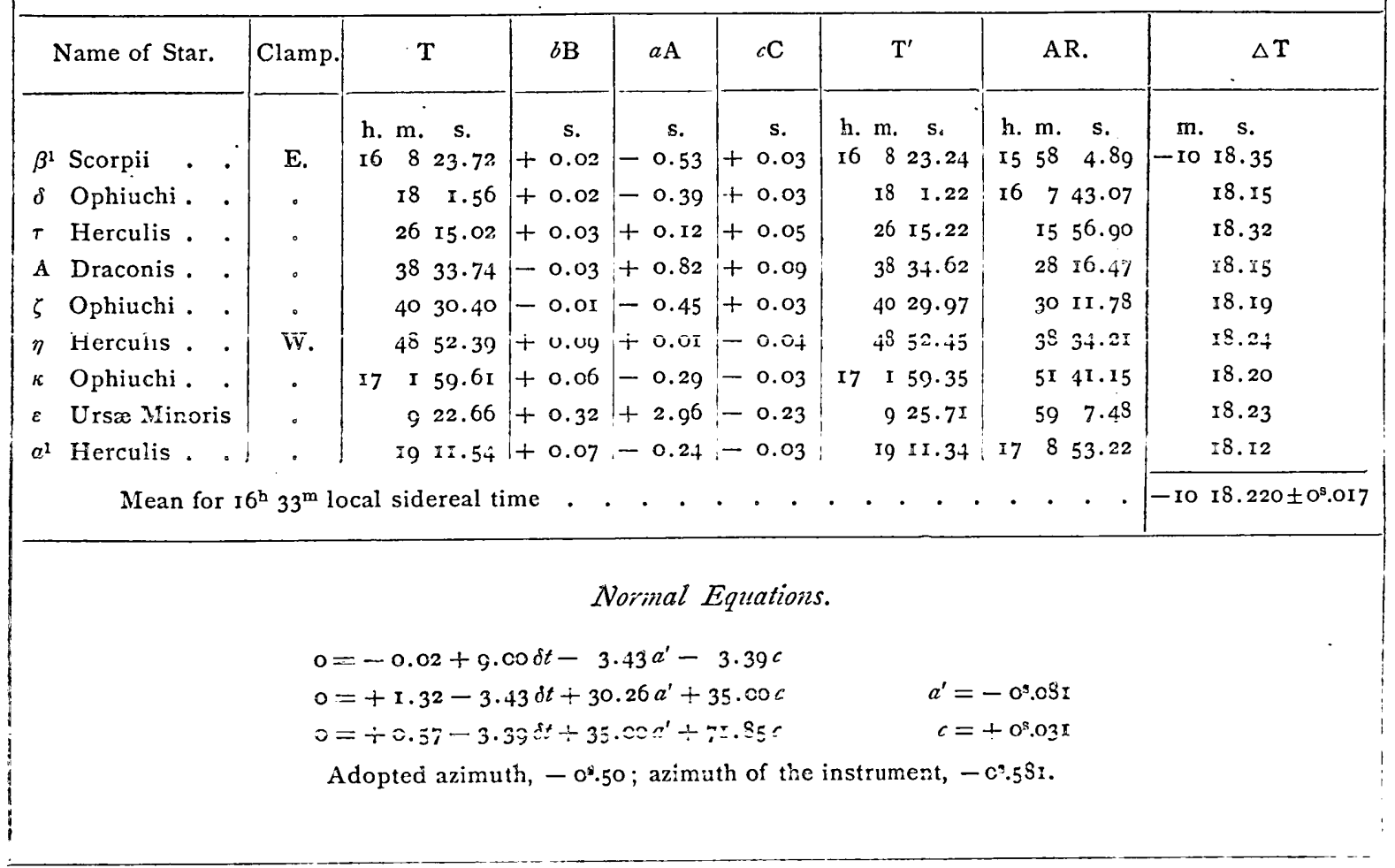


COLORADO SPRINGS, COLORADO TERRITORY, August 6, I873.

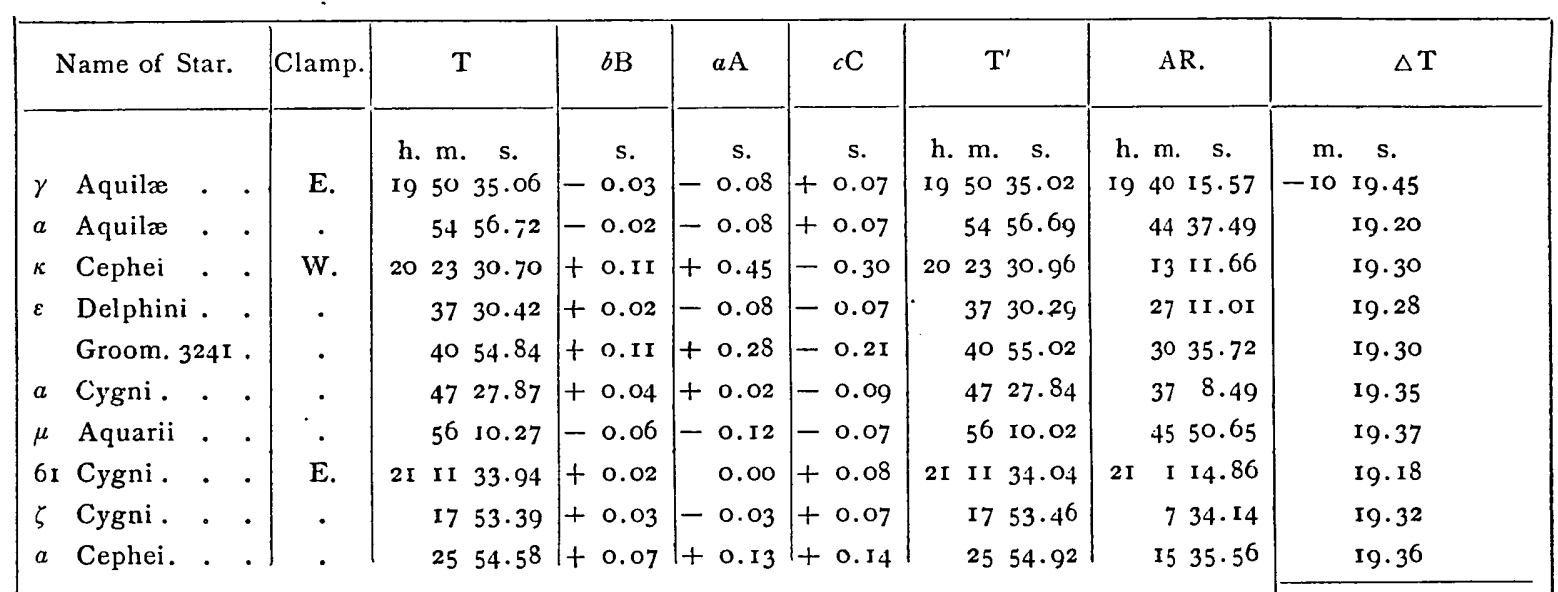

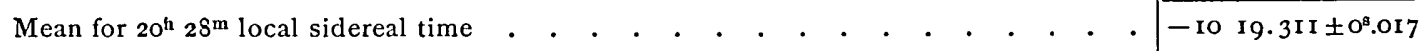

\section{Normal Equations.}

$$
\begin{array}{ll}
0=-2.07+10.00 \delta t-3.18 a-4.67 c & \\
0=+1.57-3.18 \delta t+13.31 a+17.08 c & a=-0^{8} .159 \\
0=+0.59-4.67 \delta t+17.08 a+44.96 c & c=+0^{8} .067
\end{array}
$$

SALT LAKE CITY, UTAH TERRITORY, $7 u l y 28,1873$.

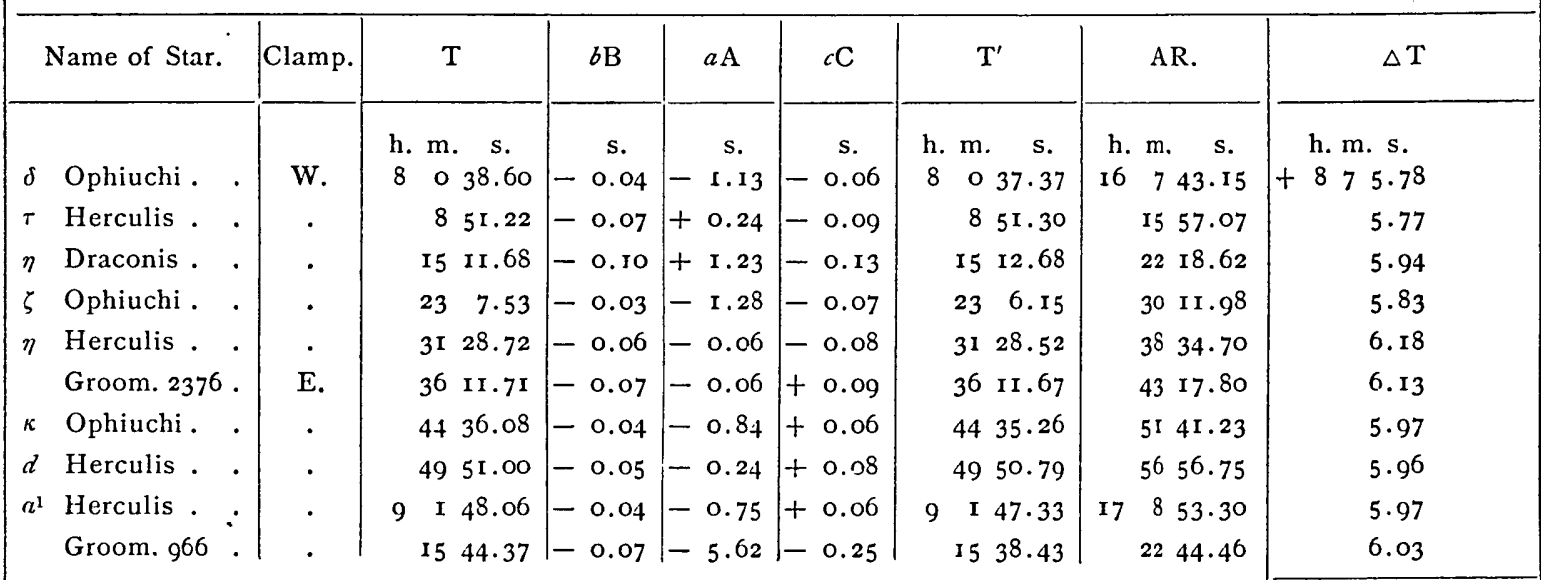

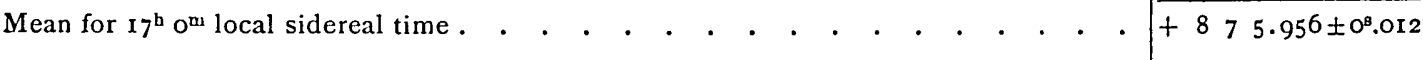

\section{Normal Equations.}

$$
\begin{array}{ll}
0=+0.15+11.00 \delta t+6.08 a-5.12 c & \delta t=-0^{8} .938 \\
0=-19.16+6.08 \delta t+14.92 a-11.02 c & a=+1^{8} .619 \\
0=+15.06-5.12 \delta t-11.02 a+31.57 c & c=-0^{8} .064
\end{array}
$$

These equations were used when $v$ Serpentis was observed; the observation is excluded for the final result on account of the doubtful position of the star. 
SALT LAKE CITY, UTAH TERRITORY, fuly 29, 1873 .

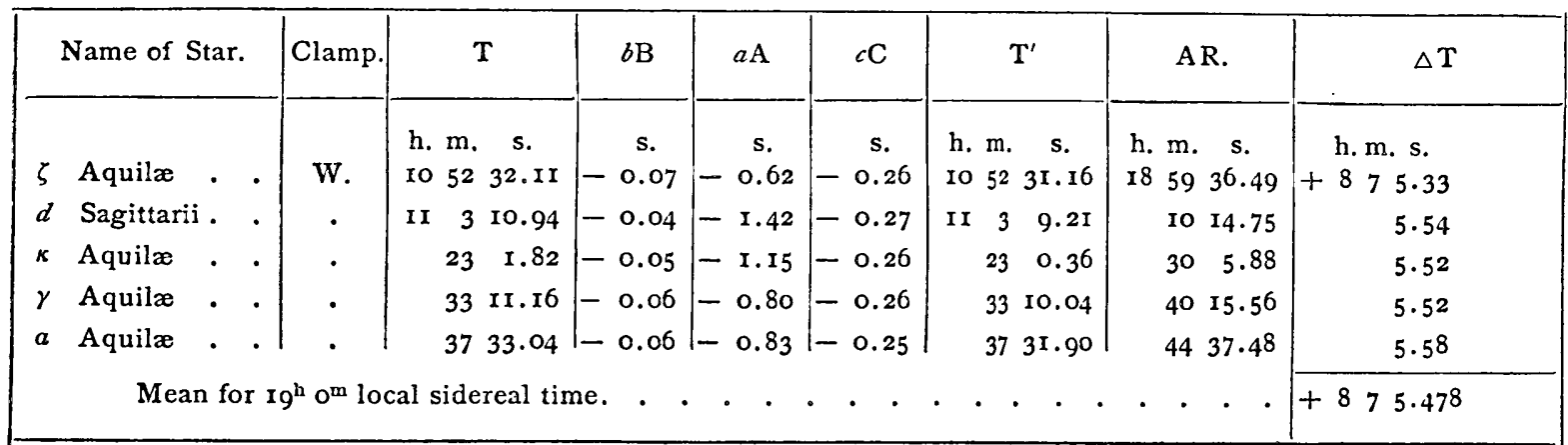

\section{Normal Equations.}

$$
\begin{array}{rlrl}
0=+\mathrm{I} . \mathrm{I} 3+5.00 \delta t+3.20 a & a & =-\mathrm{I}^{8} .530 \\
\mathrm{o}=+0.94+3.20 \delta t+2 . \mathrm{I} 9 a & c \text { (adopted) } & =+0^{8} .255
\end{array}
$$

SALT LAKE CITY, UTAH TERRITORY, $\mathfrak{f}_{u l y} 3^{\circ}, \mathrm{x} 873$.

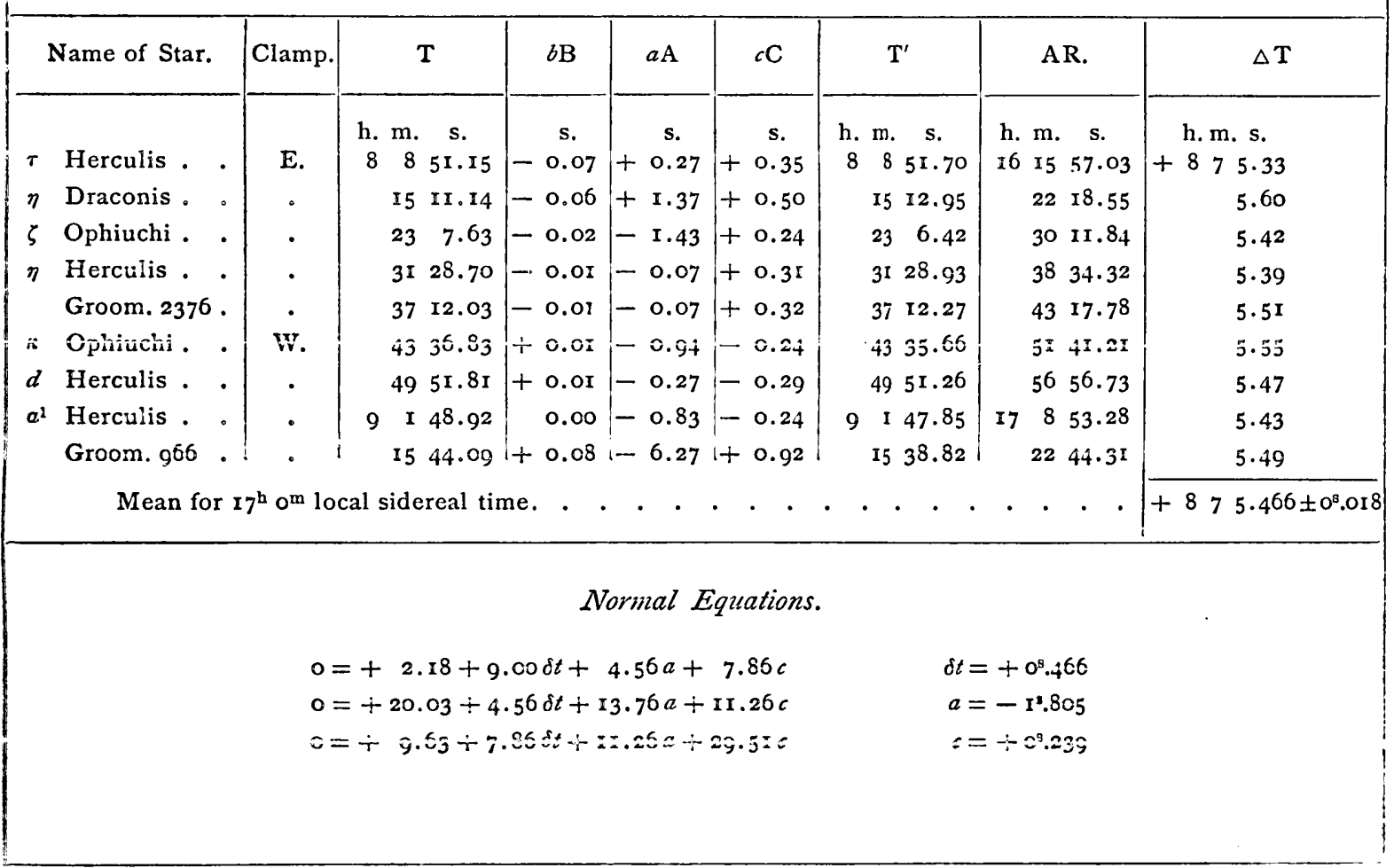


SALT LAKE CITY, UTAH TERRITORY, $\mathfrak{F}_{u l y} 30,1873$.

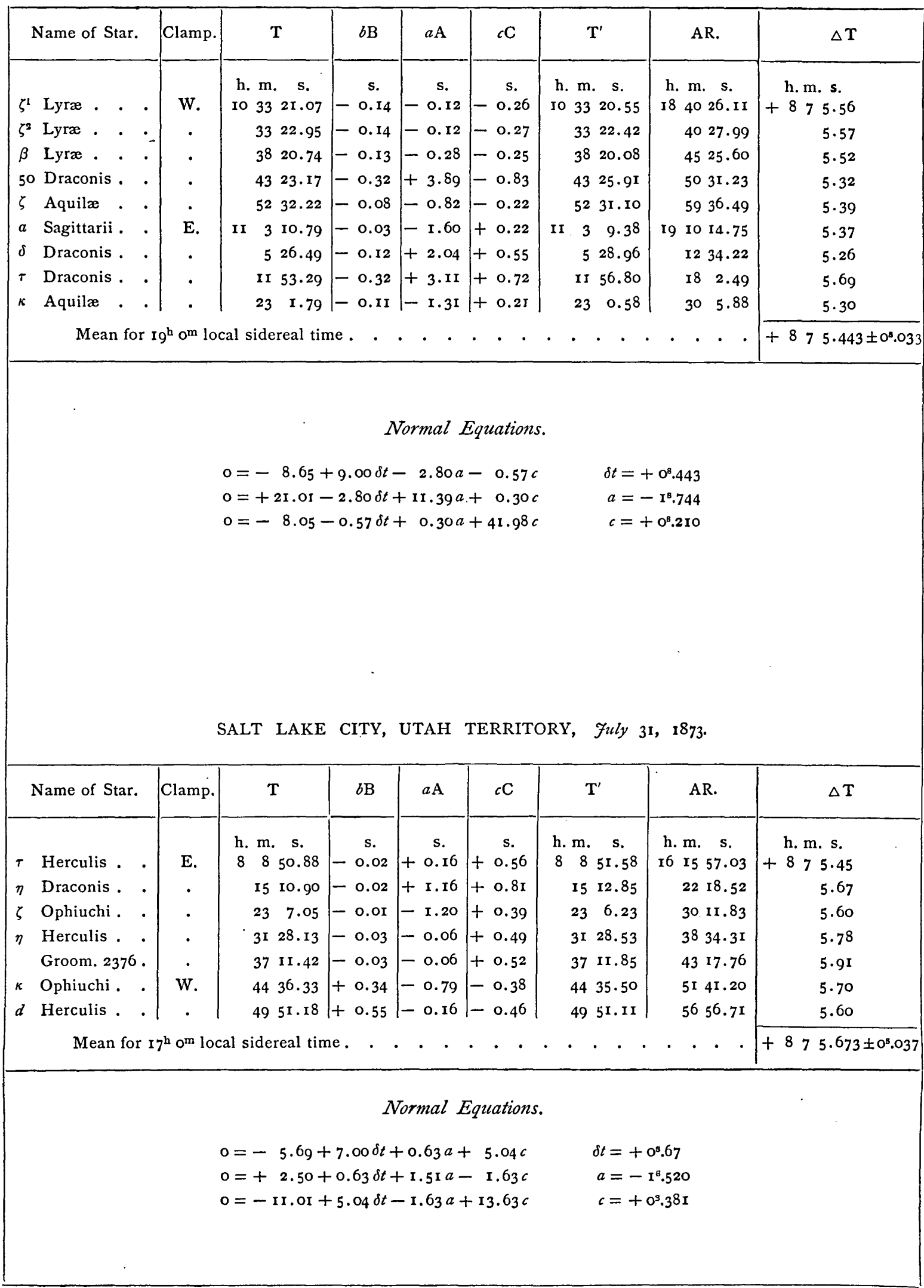


SAIT LAKE CITY, UTAY TERRITORY, $A$ ugtst 1 , 1873 .

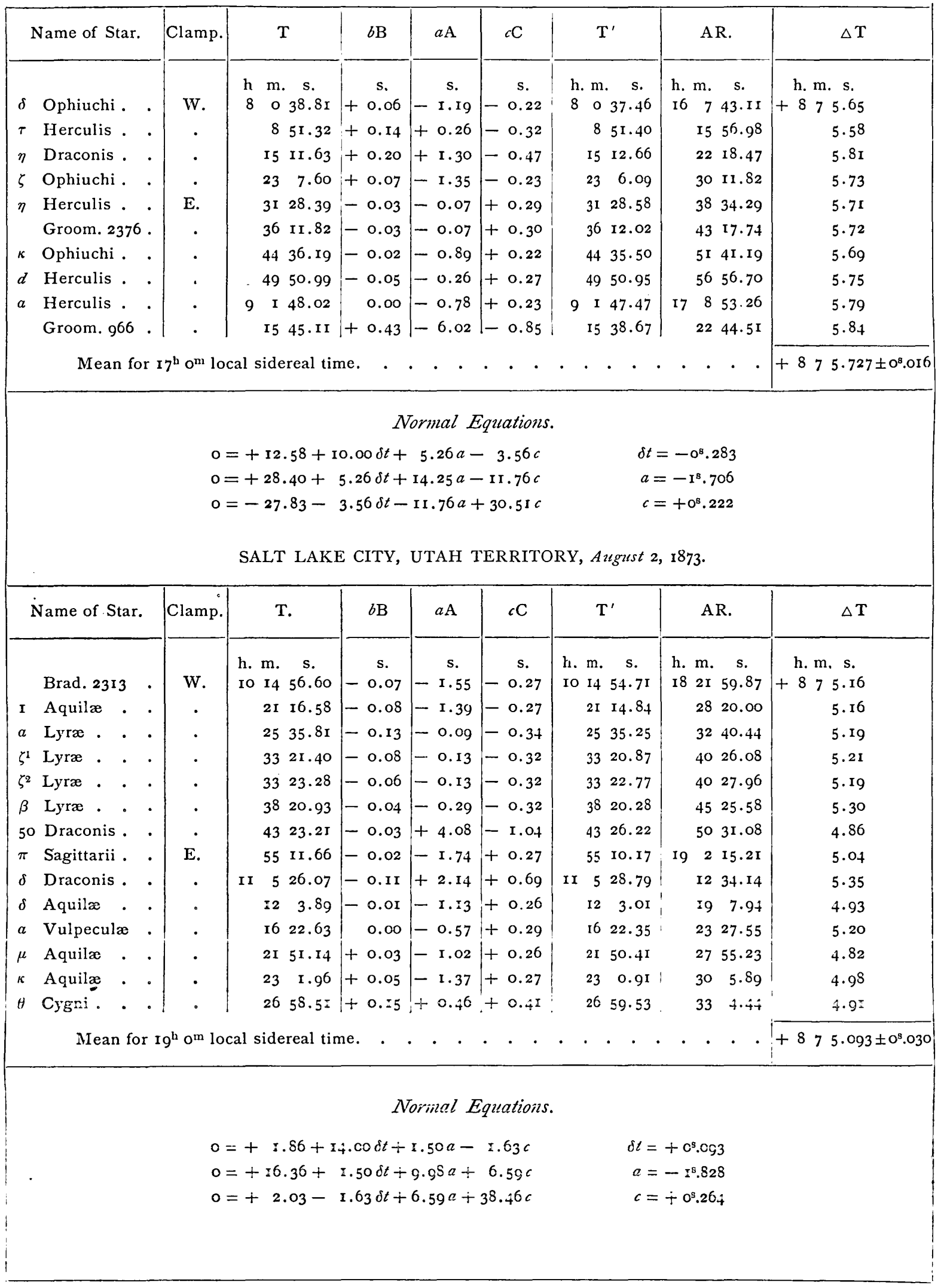


SALT LAKE CITY, UTAH TERRITORY, August 5 , I873.

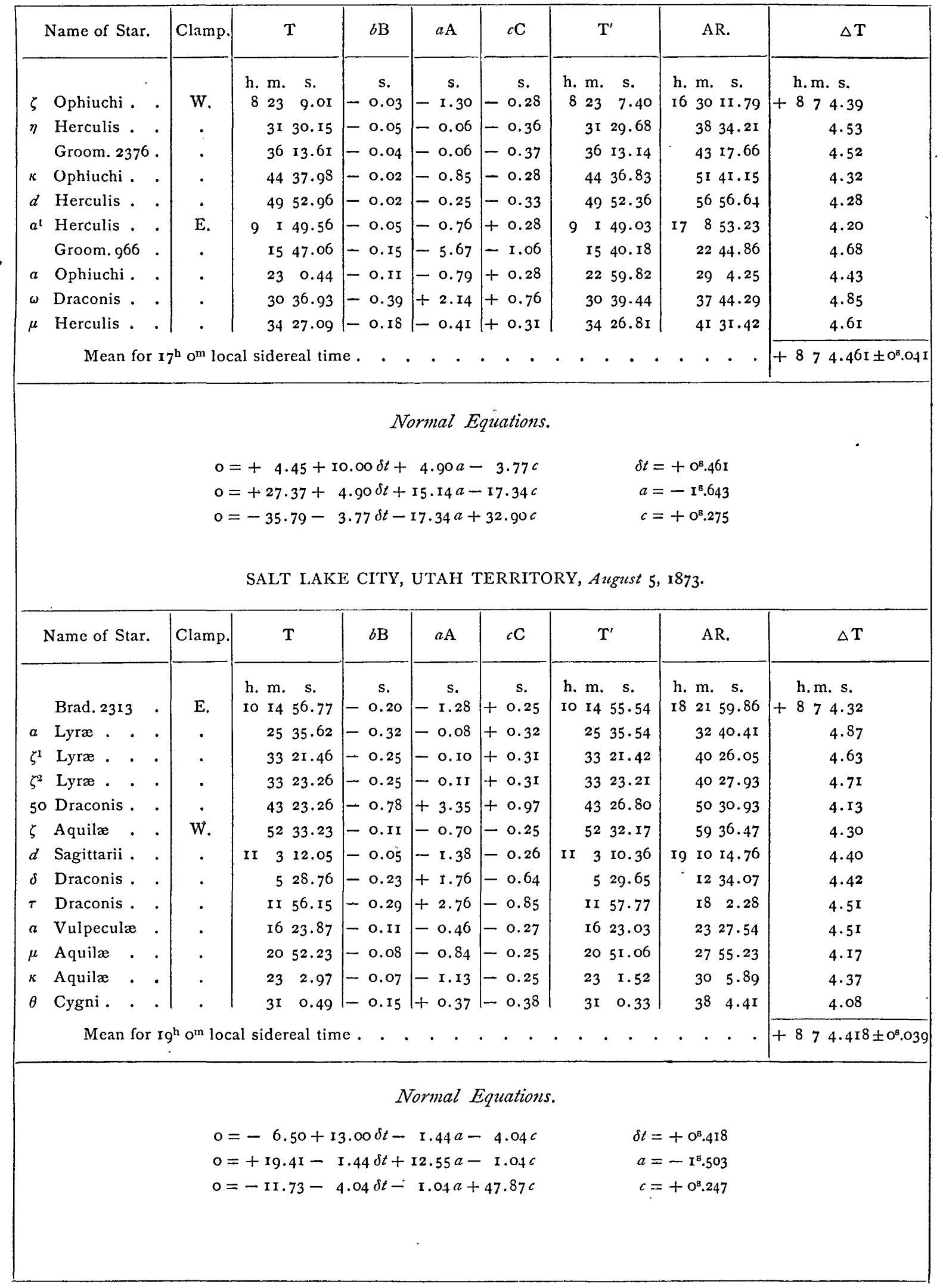


SALT LAKE CITY, UTAH TERRITORY, August 6, 1873 .

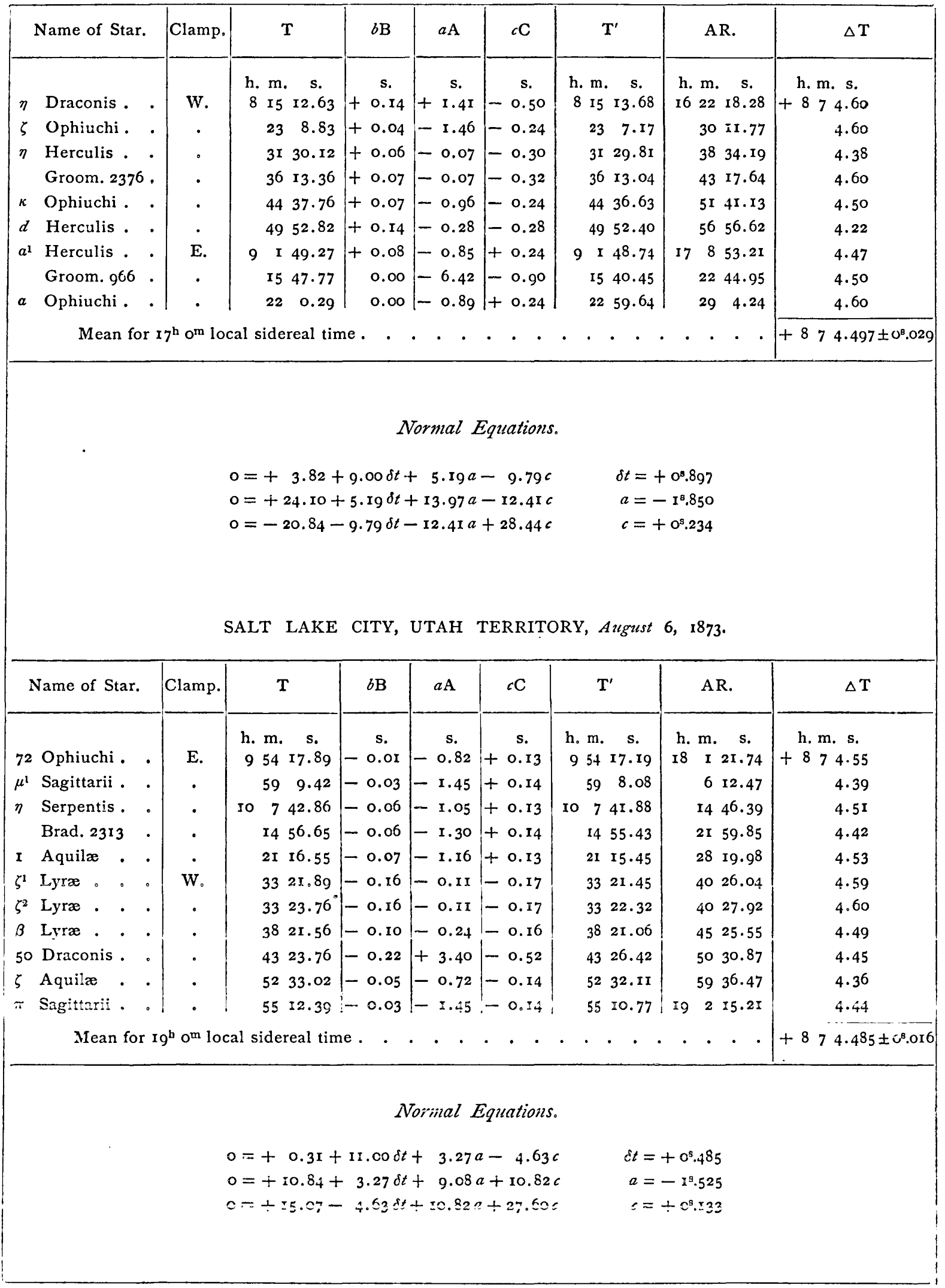


Taking the mean of the determinations of the corrections of the chronometer, the following table shows the corrections and the adopted rates of the chronometers used at Colorado Springs and Salt Lake City:

\begin{tabular}{|c|c|c|c|c|}
\hline \multicolumn{5}{|c|}{ NEGUS I49I. } \\
\hline \multirow{2}{*}{$\begin{array}{c}\text { Date. } \\
\begin{array}{c}\text { I873. } \\
\text { July } 28\end{array}\end{array}$} & \multirow{2}{*}{ 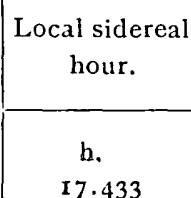 } & \multicolumn{2}{|c|}{ Correction of chronometer. } & \multirow{2}{*}{$\frac{\begin{array}{c}\text { Adopted } \\
\text { hourly rate. }\end{array}}{\text { s. }}$} \\
\hline & & h. m. & s. s. & \\
\hline 29 & 19.067 & & $11.760 \pm 0.014$ & +0.034 \\
\hline 30 & 20.000 & & $12.540 \pm 0.020$ & +0.048 \\
\hline $3 \mathrm{I}$ & 17.975 & & $13.596 \pm 0.028$ & +0.047 \\
\hline Aug. $\quad 2$ & 16.583 & & $15.745 \pm 0.020$ & +0.044 \\
\hline 4 & 19.733 & & 18 o8 $1 \pm 0.014$ & +0.007 \\
\hline 5 & 16.550 & & $\$ 8.220 \pm 0.017$ & +0.039 \\
\hline 6 & 20.467 & - 0 10 & $19.311 \pm 0.017$ & +0.060 \\
\hline \multicolumn{5}{|c|}{ NEGUS I5Ir. } \\
\hline Date. & $\begin{array}{c}\text { Local sidereal } \\
\text { hour. }\end{array}$ & Correction & of chronometer. & $\begin{array}{c}\text { Adopted } \\
\text { hourly rate. }\end{array}$ \\
\hline $\begin{array}{c}\text { I8 } 873 . \\
\text { July } 28\end{array}$ & $\begin{array}{l}\text { h. } \\
17.0\end{array}$ & $\begin{array}{r}\text { h. m. } \\
+87\end{array}$ & $\begin{array}{c}\text { s. s. } \\
5.956 \pm 0.012\end{array}$ & $\begin{array}{c}s . \\
-0.019\end{array}$ \\
\hline 29 & Ig.0 & & $5.478 \pm 0.020$ & -0.010 \\
\hline 30 & เ8.0 & & $5.455 \pm 0.026$ & +0.009 \\
\hline $3 I$ & 17.0 & & $5.673 \pm 0.037$ & +0.002 \\
\hline Aug. I & 17.0 & & $5.727 \pm 0.016$ & -0.024 \\
\hline 2 & I9.0 & & $5.093 \pm 0.030$ & -0.019 \\
\hline 5 & 18.0 & & $4.440 \pm 0.040$ & +0.002 \\
\hline 6 & t8.0 & +8 & $4.491 \pm 0.024$ & 0.000 \\
\hline
\end{tabular}


Signals for Determination of Longitude between Salt Lalie City, Utah, and Colorado Springs, Colorado Territory.

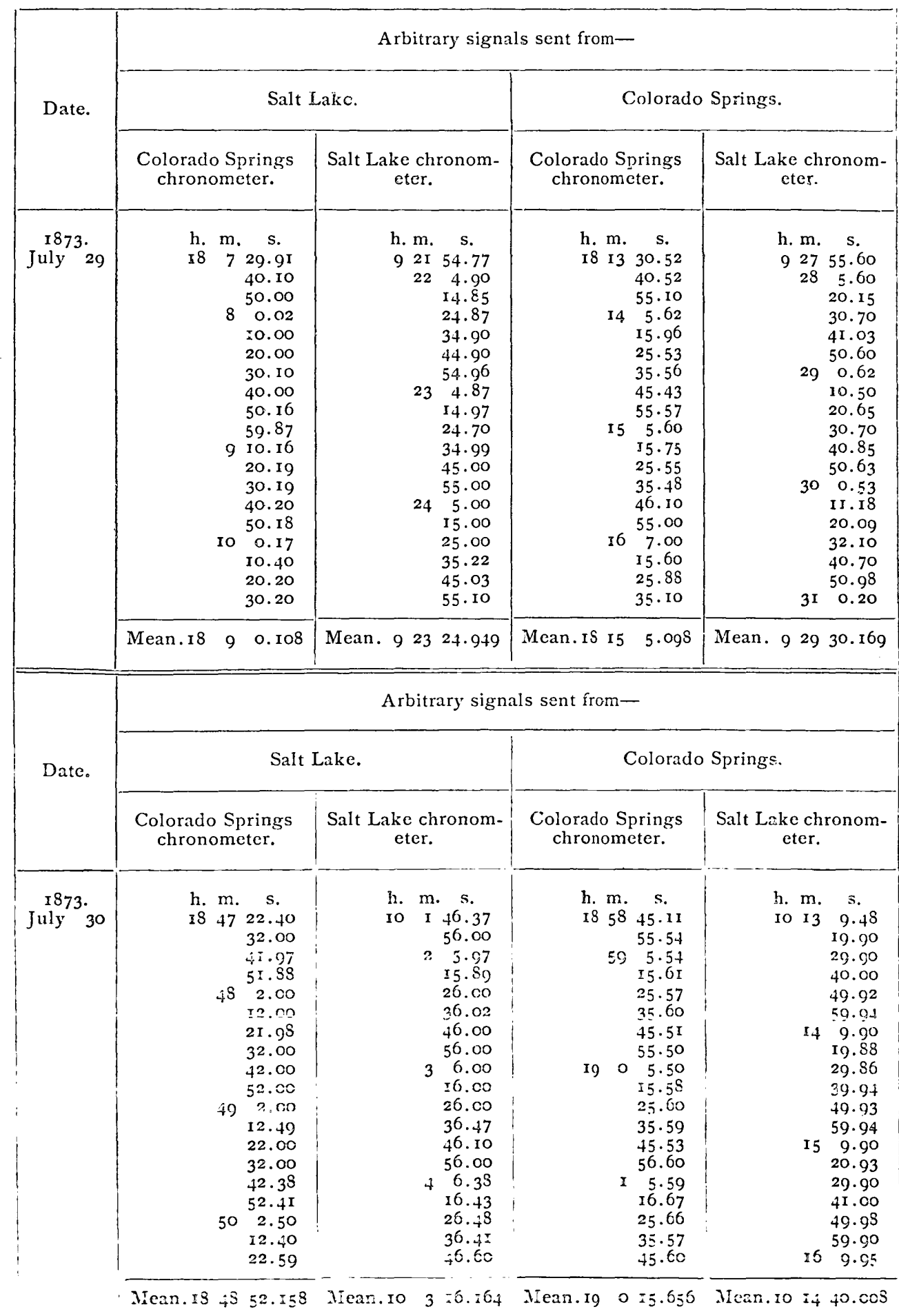


Signals for Determination of Longitude, \&c.-Continued.

\begin{tabular}{|c|c|c|c|c|}
\hline \multirow{3}{*}{ Date. } & \multicolumn{4}{|c|}{ Arbitrary signals sent from- } \\
\hline & \multicolumn{2}{|c|}{ Salt Lake. } & \multicolumn{2}{|c|}{ Colorado Springs. } \\
\hline & $\begin{array}{l}\text { Colorado Springs } \\
\text { chronometer. }\end{array}$ & $\begin{array}{c}\text { Salt Lake chronom- } \\
\text { eter. }\end{array}$ & $\begin{array}{l}\text { Colorado Springs } \\
\text { chronometer. }\end{array}$ & $\begin{array}{c}\text { Salt Lake chronom- } \\
\text { eter. }\end{array}$ \\
\hline \multirow[t]{2}{*}{$\begin{array}{l}\text { I873. } \\
\text { Augusî } 2\end{array}$} & 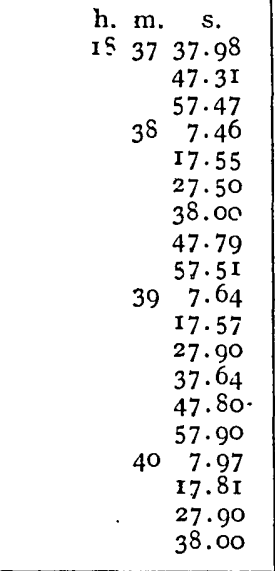 & $\begin{array}{rr}\text { h. m. } & \text { s. } \\
9 \text { 5I } & 59.00 \\
52 & 8.31 \\
& \text { I } 8.47 \\
& 28.44 \\
& 38.54 \\
& 48.50 \\
& 59.01 \\
& 8.79 \\
53 & 88.50 \\
& 18 \\
& 28.65 \\
& 38.55 \\
& 48.90 \\
& 58.66 \\
54 & 8.90 \\
18.90 \\
& 29.00 \\
& 38.82 \\
48.90 \\
& 59.00\end{array}$ & $\begin{array}{lcr}\text { h. m. } & \text { s. } \\
\text { I8 } & 42 & 55.56 \\
43 & 5.52 \\
& \text { I } 5.60 \\
& 26.53 \\
& 36.59 \\
& 46.49 \\
& 55.54 \\
& 5.60 \\
44 & 16.40 \\
& 25.60 \\
& 35.60 \\
& 45.56 \\
& 55.53 \\
45 & 8.50 \\
& 15.53 \\
& 25.59 \\
& 35.57 \\
& 45.59 \\
& 55.56\end{array}$ & $\begin{array}{rr}\text { h. m. } & \text { s. } \\
957 & 16.90 \\
& 26.86 \\
& 36.92 \\
& 47.83 \\
& 57.91 \\
& \\
58 & 7.80 \\
& 16.87 \\
& 26.93 \\
& 37.71 \\
& 46.90 \\
& 56.90 \\
& 69.86 \\
& 69 \\
& 16.87 \\
& 29.82 \\
& 36.88 \\
& 46.87 \\
& 56.85 \\
\text { Io } & 6.86 \\
& 16.90\end{array}$ \\
\hline & Mean.18 $39 \quad 7.721$ & Mean. 95328.728 & Mean.I8 $44 \quad 25 \cdot 9^{1} 4$ & Mean. $95^{8} 47.234$ \\
\hline \multirow{3}{*}{ Date. } & \multicolumn{4}{|c|}{ Arbitrary signals from- } \\
\hline & \multicolumn{2}{|c|}{ Colorado Springs. } & \multicolumn{2}{|c|}{ Salt Lake. } \\
\hline & $\begin{array}{l}\text { Colorado Springs } \\
\text { chronometer. }\end{array}$ & $\begin{array}{l}\text { Salt Lake chronom- } \\
\text { eter. }\end{array}$ & $\begin{array}{l}\text { Colorado Springs } \\
\text { chronometer. }\end{array}$ & $\begin{array}{l}\text { Salt Lake chronom- } \\
\text { eter. }\end{array}$ \\
\hline \multirow[t]{2}{*}{$\begin{array}{l}\text { I873. } \\
\text { August } 5\end{array}$} & 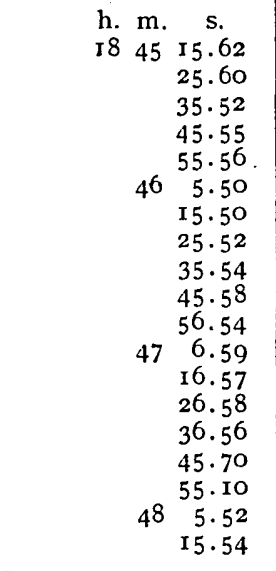 & 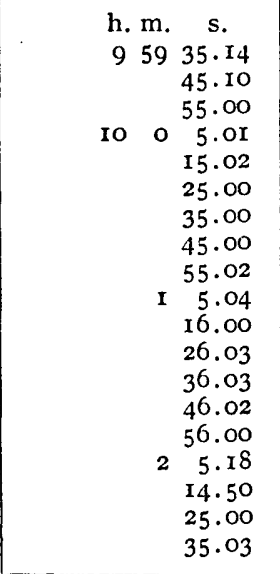 & 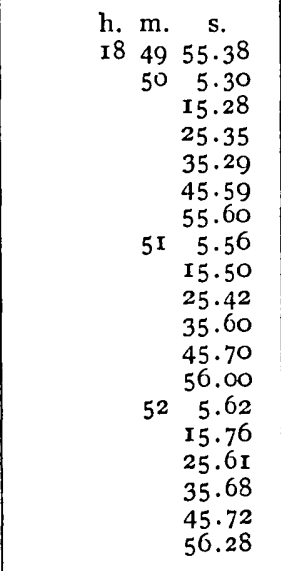 & 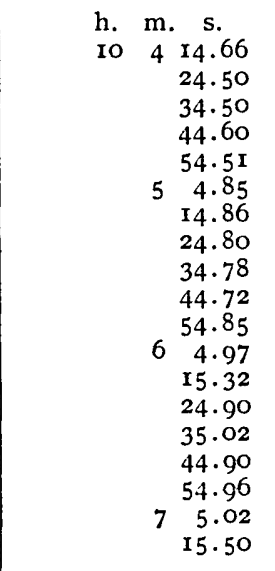 \\
\hline & Mean.18 46 45.800 & Mean.ro I 5.269 & Mean. I8 5I 25.596 & Mean.10 544.854 \\
\hline
\end{tabular}


Signals for Determination of Longitude, \&c......Continued.

\begin{tabular}{|c|c|c|c|c|}
\hline \multirow{3}{*}{ Date. } & \multicolumn{4}{|c|}{ Arbitrary signals sent from- } \\
\hline & \multicolumn{2}{|c|}{ Salt Lake. } & \multicolumn{2}{|c|}{ Colorado Springs. } \\
\hline & $\begin{array}{l}\text { Colorado Springs } \\
\text { chronometer. }\end{array}$ & $\begin{array}{c}\text { Salt Lake chronom- } \\
\text { eter. }\end{array}$ & $\begin{array}{l}\text { Colorado springs } \\
\text { chronometer. }\end{array}$ & $\begin{array}{l}\text { Salt Lake chronom- } \\
\text { eter. }\end{array}$ \\
\hline $\begin{array}{l}\text { I873. } \\
\text { Aug. } 6\end{array}$ & $\begin{array}{cc}\text { h. m. } & \text { s. } \\
18 \text { r5 } & 35.24 \\
& 45.18 \\
& 55.00 \\
\text { I6 } & 5.40 \\
& 15.24 \\
& 25.19 \\
& 35.03 \\
& 45.29 \\
& 55.18 \\
\text { I7 } & 5.30 \\
& 15.47 \\
& 25.48 \\
& 35.00 \\
& 45.40 \\
& 55.30 \\
18 & 5.60 \\
15.59 \\
\\
& 25.64 \\
& 35.61\end{array}$ & $\begin{array}{rr}\text { h. m. } & \text { s. } \\
9 \text { 29 } & 53.60 \\
30 & 3.59 \\
& 13.50 \\
& 23.80 \\
& 33.65 \\
& 43.53 \\
& 53.50 \\
31 & 3.65 \\
13.53 \\
\\
23.69 \\
33.86 \\
43.87 \\
53.50 \\
32 \\
3.80 \\
13.65 \\
23.98 \\
33.97 \\
44.00 \\
54.03\end{array}$ & $\begin{array}{cc}\text { h. m. s. } \\
\text { I8 } 24 \text { I5.60 } \\
25.55 \\
35.61 \\
\\
45.60 \\
\\
55.59 \\
25 \quad 5.61 \\
15.12 \\
25.61 \\
\\
34.60 \\
44.61 \\
44.60 \\
26 \\
4.60 \\
15.68 \\
25.60 \\
35.00 \\
45.10 \\
55.62 \\
27.60 \\
56.53\end{array}$ & 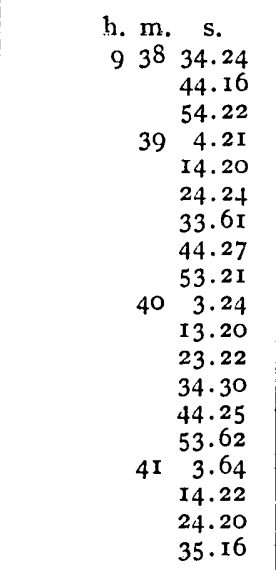 \\
\hline & Mean.18 I7 5.323 & Mean.931 23.72I & Mean.18 2545.360 & Mean. $940 \quad 3.969$ \\
\hline
\end{tabular}

July 30, the breaks for the seconds of Salt Lake and Colorado Springs coincide. By measuring the differences where I find the full minute, I develop the fact that the Colorado Springs chronometer breaks $0^{8} .1$ Io later than the Salt Lake break; therefore the mean of the signals sent from Salt Lake and received on the Colorado Springs chronograph is, $18^{\mathrm{b}} 4^{8^{\mathrm{m}}} 5^{2^{\mathrm{B}} .048 .}$

The same happens on August 2. The Colorado Springs chronometer is $0^{8} .095$ later than the Salt Lake chronometer on the Colorado Springs chronograph; therefore the mean is, $18^{\mathrm{h}} 39^{\mathrm{m}} 7^{\mathrm{s}} .626$. 
Final Results for Longitude.

\begin{tabular}{|c|c|c|c|c|c|c|c|}
\hline Signals sent from- & Stations of record. & $\begin{array}{l}\text { Mean of sig- } \\
\text { nals sent and } \\
\text { received. }\end{array}$ & $\begin{array}{l}\text { Time-correc- } \\
\text { tion. }\end{array}$ & $\begin{array}{c}\text { Corrected } \\
\text { time. }\end{array}$ & $\begin{array}{c}\text { Difference of } \\
\text { longitude. }\end{array}$ & $\begin{array}{c}\text { Double } \\
\text { wave-time. }\end{array}$ & Means. \\
\hline $\begin{array}{c}\text { 1873. } \\
\mathcal{F}_{f u l y} 29 . \\
\text { Salt Lake } \ldots .\{\end{array}$ & $\begin{array}{l}\text { Colo. Springs chron. } \\
\text { Salt Lake chron. . - }\end{array}$ & $\begin{array}{ccc}\text { h. m. } & \text { s. } \\
\text { I8 } & 9 & 0.1 \text { I } \\
& & \\
9 & 23 & 24.95\end{array}$ & $\begin{array}{r}\text { h. m. s. } \\
-0 \text { 10 I1:72 } \\
+875.46\end{array}$ & 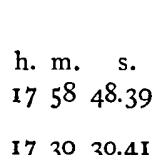 & $\begin{array}{l}\text { h. m. } \mathrm{s} . \\
\text { o } 28 \\
17.98\end{array}$ & s. & s. \\
\hline Colorado Springs . \{ & $\begin{array}{l}\text { Colo. Springs chron. } \\
\text { Salt Lake chron. . }\end{array}$ & $\begin{array}{lll}18 & 15 & 5.10 \\
9 & 29 & 30.17\end{array}$ & $\begin{array}{l}-0 \text { 10 } 11.72 \\
+875.46\end{array}$ & $\begin{array}{l}18453.3^{8} \\
173635.63\end{array}$ & 17.75 & 0.23 & 17.865 \\
\hline $\begin{array}{c}\mathcal{f}^{\prime} u l y \\
30 . \\
\text { Salt Lake } . . .\{\end{array}$ & $\begin{array}{l}\text { Colo. Springs chron. } \\
\text { Salt Lake chron. - . }\end{array}$ & $\begin{array}{l}184852.05 \\
10 \quad 3 \quad 16.16\end{array}$ & $\begin{array}{r}-01012.48 \\
+875.46\end{array}$ & $\begin{array}{l}\text { I8 } 38 \quad 39.57 \\
\text { I8 I0 } 21.62\end{array}$ & I 7.95 & & \\
\hline Colorado Springs . \{ & $\begin{array}{l}\text { Colo. Springs chron. } \\
\text { Salt Lake chron. . - }\end{array}$ & $\begin{array}{lll}19 & 0 & 15.66 \\
10 & 14 & 40.01\end{array}$ & 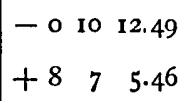 & $\begin{array}{l}1850 \quad 3.17 \\
1821 \quad 45.47\end{array}$ & I 7.70 & 0.25 & 17.825 \\
\hline Salt Lake ... & $\begin{array}{l}\text { Colo. Springs chron. } \\
\text { Salt Lake chron. . - }\end{array}$ & $\begin{array}{rrr}18 & 39 & 7.63 \\
9 & 53 & 28.73\end{array}$ & $\begin{array}{l}-01015.83 \\
+875.11\end{array}$ & $\begin{array}{lll}18 & 28 & 51.80 \\
18 & \circ & 33.84\end{array}$ & I 7.96 & & \\
\hline Colorado Springs . \{ & $\begin{array}{l}\text { Colo. Springs chron. } \\
\text { Salt Lake chron. - - }\end{array}$ & $\begin{array}{rrr}18 & 44 & 25.91 \\
9 & 58 & 47.24\end{array}$ & $\begin{array}{l}-01015.83 \\
+875.11\end{array}$ & $\begin{array}{lll}18 & 34 & 10.08 \\
18 & 5 & 52.35\end{array}$ & 17.73 & 0.23 & I 7.845 \\
\hline Colorado Springs.\{ & $\begin{array}{l}\text { Colo. Springs chron. } \\
\text { Salt Lake chron. . . }\end{array}$ & 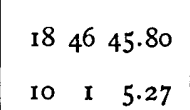 & $\begin{array}{l}-0 \text { 10 } 18.30 \\
+874.44\end{array}$ & $\begin{array}{lll}18 & 36 & 27.50 \\
18 & 8 & 9.71\end{array}$ & I 7.79 & & \\
\hline $\begin{array}{c}\text { Salt Lake... } \\
\text { August } 6 .\end{array}$ & $\begin{array}{l}\text { Colo. Springs chron. } \\
\text { Salt Lake chron. . }\end{array}$ & $\begin{array}{lrr}18 & 51 & 25.60 \\
10 & 5 & 44.85\end{array}$ & $\begin{array}{l}-0 \text { 10 } 18.31 \\
+874.44\end{array}$ & $\begin{array}{lrr}\mathrm{I} 8 & 4 \mathrm{I} & 7.29 \\
\mathrm{I} 8 & \mathrm{I} 2 & 49.29\end{array}$ & 18.00 & 0.21 & 1 7.895 \\
\hline Salt Lake ... & $\begin{array}{l}\text { Colo. Springs chron. } \\
\text { Salt Lake chron. - }\end{array}$ & $\begin{array}{rrr}18 & 17 & 5 \cdot 32 \\
9 & 31 & 23 \cdot 72\end{array}$ & $\begin{array}{l}-0 \text { 10 } 19.17 \\
+874.49\end{array}$ & $\begin{array}{lll}18 & 6 & 46.15 \\
17 & 38 & 28.21\end{array}$ & 17.94 & & \\
\hline Colorado Springs . \{ & $\begin{array}{l}\text { Colo. Springs chron. } \\
\text { Salt Lake chron. . - }\end{array}$ & $\begin{array}{rrr}18 & 25 & 45 \cdot 3^{6} \\
9 & 40 & 3.97\end{array}$ & $\begin{array}{l}-0 \text { 10 } 19.18 \\
+874.49\end{array}$ & $\begin{array}{l}181526.18 \\
1747 \quad 8.46\end{array}$ & 17.72 & 0.22 & 17.830 \\
\hline
\end{tabular}


Mean Places of Sturs for 18\%3.0, ased for Determination of Latitud? of Coloralo Springs, Colorado Territory.

\begin{tabular}{|c|c|c|c|c|c|c|c|}
\hline $\begin{array}{l}\text { No. of } \\
\text { pair. }\end{array}$ & $\begin{array}{l}\text { No. in } \\
\text { B. A. C. }\end{array}$ & $\begin{array}{c}\text { Right } \\
\text { ascension. }\end{array}$ & Declination. & $\begin{array}{l}\text { No. of } \\
\text { pair. }\end{array}$ & $\begin{array}{l}\text { No. in } \\
\text { B. A. C. }\end{array}$ & $\begin{array}{c}\text { Right } \\
\text { ascension. }\end{array}$ & Declination. \\
\hline $\mathbf{I}$ & $\begin{array}{l}5587 \\
5628\end{array}$ & 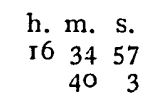 & $\begin{array}{ccc}\circ & 1 & \prime \prime \\
12 & 38 & 35.4 \\
64 & 49 & 48.4\end{array}$ & 25 & $\begin{array}{l}7297 \\
7320\end{array}$ & $\begin{array}{rrr}\text { h. } & \text { m. } & \text { s. } \\
20 & 55 & 5 \\
& 58 & 9\end{array}$ & $\begin{array}{ccc}\circ & \prime & \prime \prime \\
39 & 45 & 23.86 \\
38 & 9 & 23.70\end{array}$ \\
\hline 2 & $\begin{array}{l}5747 \\
5775\end{array}$ & 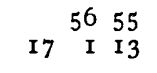 & $\begin{array}{rrr}33 & 45 & 14.08 \\
43 & 59 & 9.46\end{array}$ & 26 & $\begin{array}{l}736 \mathrm{r} \\
740 \mathrm{r}\end{array}$ & 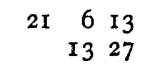 & $\begin{array}{lll}22 & 33 & 45.2 \\
55 & \mathrm{I} 5 & 54.42\end{array}$ \\
\hline 3 & $\begin{array}{l}5790 \\
5834\end{array}$ & $\begin{array}{r}338 \\
\text { 10 } 38\end{array}$ & $\begin{array}{lll}40 & 40 & 59.86 \\
36 & 57 & 13.04\end{array}$ & 27 & $\begin{array}{l}7444 \\
7489\end{array}$ & $\begin{array}{ll}\text { I } 8 & 55 \\
27 & \text { II }\end{array}$ & $\begin{array}{rrr}25 & 37 & 43.49 \\
52 & 3 & 36.28\end{array}$ \\
\hline 4 & $\begin{array}{l}5871 \\
5927\end{array}$ & $\begin{array}{rr}16 & 46 \\
26 & 7\end{array}$ & $\begin{array}{lll}46 & 21 & 58.58 \\
31 & 15 & 15.10\end{array}$ & 28 & $\begin{array}{l}7505 \\
7521\end{array}$ & $\begin{array}{ll}29 & 35 \\
31 & 52\end{array}$ & $\begin{array}{lll}37 & 57 & 56 \cdot 32 \\
39 & 50 & 37.76\end{array}$ \\
\hline 5 & $\begin{array}{l}5978 \\
599 I\end{array}$ & $\begin{array}{ll}33 & 41 \\
36 & 16\end{array}$ & $\begin{array}{rrr}61 & 58 & 18.9 \\
16 & 0 & 44.7\end{array}$ & 29 & $\begin{array}{l}7554 \\
7566\end{array}$ & $\begin{array}{ll}36 & 28 \\
38 & 10\end{array}$ & $\begin{array}{lll}40 & 13 & 45.02 \\
37 & 42 & 10.92\end{array}$ \\
\hline $\begin{array}{l}6 \\
7\end{array}$ & $\begin{array}{l}6079 \\
6110 \\
6157\end{array}$ & $\begin{array}{r}5120 \\
5657 \\
18 \quad 320\end{array}$ & $\begin{array}{lll}56 & 53 & 35 \cdot 36 \\
20 & 50 & 5 \cdot 7 \\
20 & 47 & 46.2\end{array}$ & 30 & $\begin{array}{l}7621 \\
7641\end{array}$ & $\begin{array}{ll}46 & \text { I } 2 \\
50 & 45\end{array}$ & $\begin{array}{lll}66 & 12 & 8.4 \\
\text { II } & 28 & 27.5\end{array}$ \\
\hline 8 & $\begin{array}{l}6238 \\
6255\end{array}$ & $\begin{array}{rr}\text { I6 } & 4 \\
\text { I } 8 & 18\end{array}$ & $\begin{array}{lll}28 & 48 & 39.4 \\
49 & 3 & 28.30\end{array}$ & $3 I$ & $\begin{array}{l}7683 \\
7733\end{array}$ & $\begin{array}{r}5748 \\
22 \quad 430\end{array}$ & $\begin{array}{lll}57 & 23 & 17.18 \\
20 & 21 & 16.4\end{array}$ \\
\hline $\begin{array}{r}9 \\
10\end{array}$ & $\begin{array}{l}6357 \\
6365 \\
6391\end{array}$ & $\begin{array}{ll}33 & 55 \\
35 & 54 \\
40 & \mathrm{I} 4\end{array}$ & $\begin{array}{rrr}39 & 33 & 26.28 \\
38 & 15 & 1.54 \\
39 & 28 & 51.50\end{array}$ & 32 & $\begin{array}{l}7757 \\
7825\end{array}$ & $\begin{array}{rl}7 & 50 \\
20 & 17\end{array}$ & $\begin{array}{rrr}27 & 58 & 46.49 \\
49 & 45 & 25.22 \\
-0 & & \\
- & 40 & 9.8\end{array}$ \\
\hline I I & $\begin{array}{l}6468 \\
6475\end{array}$ & $\begin{array}{ll}50 & 13 \\
51 & 28\end{array}$ & $\begin{array}{lll}33 & 48 & 27 \cdot 32 \\
43 & 46 & 47.30\end{array}$ & $\begin{array}{l}33 \\
34\end{array}$ & $\begin{array}{l}7857 \\
7874\end{array}$ & $\begin{array}{l}2544 \\
2844\end{array}$ & $\begin{array}{rrrr}78 & 8 & 18.1 \\
78 & \text { 10 } & 19.9\end{array}$ \\
\hline 12 & $\begin{array}{l}6520 \\
6571\end{array}$ & 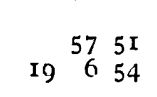 & $\begin{array}{rrr}46 & 45 & 20.56 \\
31 & 4 & 22.42\end{array}$ & 35 & $\begin{array}{r}7880 \\
\text { Gr.3873 } \\
793 \mathrm{I}\end{array}$ & $\begin{array}{rr}30 & 13 \\
39 & 8 \\
38 & 21\end{array}$ & $\begin{array}{rrr}38 & 58 & 39.82 \\
38 & 32 & 7 \cdot 36 \\
38 & 48 & 1,90\end{array}$ \\
\hline 13 & $\begin{array}{l}6586 \\
66 \text { I } 5\end{array}$ & $\begin{array}{rr}9 & 17 \\
13 & 44\end{array}$ & $\begin{array}{rrr}65 & 45 & 57.5 \\
12 & 8 & 35.4\end{array}$ & 36 & $\begin{array}{l}7951 \\
7990\end{array}$ & $\begin{array}{l}41 \text { I } 8 \\
47 \quad 55\end{array}$ & $\mid \begin{array}{rrr}-4 & 53 & 13.7 \\
82 & 28 & 47.5\end{array}$ \\
\hline 14 & $\begin{array}{l}6652 \\
668 I\end{array}$ & $\begin{array}{ll}\text { 19 } & 49 \\
23 & 29\end{array}$ & $\begin{array}{rrr}z 0 & 1 & 21.6 \\
57 & 46 & 18.90\end{array}$ & $\begin{array}{l}37 \\
38\end{array}$ & $\begin{array}{l}8003 \\
8039 \\
8077\end{array}$ & 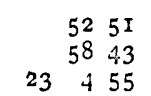 & $\begin{array}{lrr}\text { II } & 3 & 3 \cdot 3 \\
66 & 3 \mathrm{I} & 29 \cdot 0 \\
66 & 33 & 9 \cdot 5\end{array}$ \\
\hline I5 & $\begin{array}{l}6698 \\
6720\end{array}$ & $\begin{array}{rr}27 & 3 \\
30 & 43\end{array}$ & $\begin{array}{lll}34 & \text { II } & 3 \cdot 32 \\
43 & 40 & 2 \cdot 52\end{array}$ & 39 & $\begin{array}{l}8147 \\
8188\end{array}$ & $\begin{array}{ll}\text { I6 } & 26 \\
24 & 11\end{array}$ & $\begin{array}{lll}\text { I9 } & 51 & 47.4 \\
57 & 50 & 55.08\end{array}$ \\
\hline 16 & $\begin{array}{l}6731 \\
6784\end{array}$ & $\begin{array}{ll}32 & 42 \\
4 I \quad 36\end{array}$ & $\begin{array}{rrr}44 & 24 & 56.30 \\
33 & 26 & 0.74\end{array}$ & 40 & Gr.4IIO & $322 \mathrm{I}$ & $\begin{array}{lll}57 & 57 \quad 5.58\end{array}$ \\
\hline I 7 & $\begin{array}{l}6819 \\
6852\end{array}$ & $\begin{array}{ll}46 & 42 \\
51 & 19\end{array}$ & $\begin{array}{lll}18 & 20 & 49.49 \\
59 & 22 & 23.28\end{array}$ & $\begin{array}{l}4 \mathrm{I} \\
42\end{array}$ & $\begin{array}{l}8296 \\
8310 \\
8317\end{array}$ & $\begin{array}{rr}45 & 57 \\
48 & 3 \\
49 & 12\end{array}$ & $\begin{array}{lll}20 & 57 & 54.22 \\
56 & 47 & 34.04 \\
56 & 42 & 18.74\end{array}$ \\
\hline$x 8$ & 6863 & $525 \mathrm{x}$ & $57 \quad 54 \quad 55$. & 43 & $\begin{array}{r}\text { Gr.t216 } \\
8374\end{array}$ & $0 \quad \begin{array}{rr}57 & 39 \\
0 & I\end{array}$ & $\begin{array}{rrr}49 & 9 & 47 \cdot 30 \\
28 & 19 & 15.15\end{array}$ \\
\hline & 6918 & 20 I 40 & 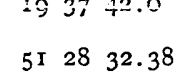 & 44 & $\begin{array}{l}28 \\
67\end{array}$ & $\begin{array}{rr}0 & 35 \\
14 & 26\end{array}$ & $\begin{array}{llr}40 & 20 & 3.36 \\
37 & 15 & 53.68\end{array}$ \\
\hline 19 & $69+4$ & $64 \mathrm{I}$ & $\begin{array}{lll}26 & 6 & 2.7\end{array}$ & 45 & $\begin{array}{r}87 \\
105\end{array}$ & $\begin{array}{ll}\text { I8 } & 54 \\
22 & 45\end{array}$ & $\begin{array}{lll}1 & 14 & 10.80 \\
70 & 19 & 5.8\end{array}$ \\
\hline 20 & $\begin{array}{l}6963 \\
6998\end{array}$ & $\begin{array}{r}926 \\
1346\end{array}$ & $\begin{array}{lll}42 & 59 & 40.52 \\
34 & 35 & 13.28\end{array}$ & 46 & $\begin{array}{l}\text { I2I } \\
178\end{array}$ & $\begin{array}{l}2447 \\
3452\end{array}$ & $\begin{array}{lll}53 & 49 & 15.16 \\
23 & 55 & 57.42\end{array}$ \\
\hline $2 I$ & $\begin{array}{l}7022 \\
700 ́:\end{array}$ & $\begin{array}{l}1740 \\
2252\end{array}$ & $\begin{array}{rrr}3951 & 4.58 \\
38 & : 27.56\end{array}$ & $\begin{array}{l}47 \\
48\end{array}$ & $\begin{array}{r}201 \\
\text { Gr. } 137 \\
250\end{array}$ & $\begin{array}{rr}35 & 4 \\
39 & 0 \\
49 & 10\end{array}$ & $\begin{array}{lll}54 & 3 \mathrm{I} & 33.26 \\
54 & 36 & 37.4 \\
22 & 56 & 25.7 \mathrm{I}\end{array}$ \\
\hline 22 & $\begin{array}{l}708_{4} \\
7 \times 0:\end{array}$ & $\begin{array}{ll}26 & 10 \\
28 & 29\end{array}$ & $\begin{array}{rrr}36 & 30 & 32.70 \\
4 I & 2 & 24.5 S\end{array}$ & 49 & $\begin{array}{l}285 \\
330\end{array}$ & $\begin{array}{r}5552 \\
=\quad 28\end{array}$ & $\begin{array}{lrr}31 & 7 & 18.84 \\
46 & 33 & 49.88\end{array}$ \\
\hline 23 & 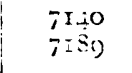 & $\begin{array}{lr}32 & 5 \mathrm{r} \\
39 & \mathrm{~S}\end{array}$ & $\begin{array}{lll}20 & 45 & 23.4 \\
56 & 55 & 44.74\end{array}$ & 50 & $\begin{array}{l}357 \\
+7=\end{array}$ & $\begin{array}{r}549 \\
22 \quad 30\end{array}$ & $\begin{array}{lll}31 & 24 & 3.54 \\
4 & 21 & 4.50\end{array}$ \\
\hline 24 & $\begin{array}{l}, 2+3 \\
7256\end{array}$ & $\begin{array}{l}533 \\
4938\end{array}$ & $\begin{array}{lll}50 & +8 & 7.5 \\
27 & 3+33.03\end{array}$ & $5^{I}$ & 517 & $\begin{array}{l}28+2 \\
34 \quad 29\end{array}$ & $\begin{array}{ccc}45 & +23.02 \\
29 & 2+13.5\end{array}$ \\
\hline
\end{tabular}


Observations for Latitude.-Station, Colorado Springs.

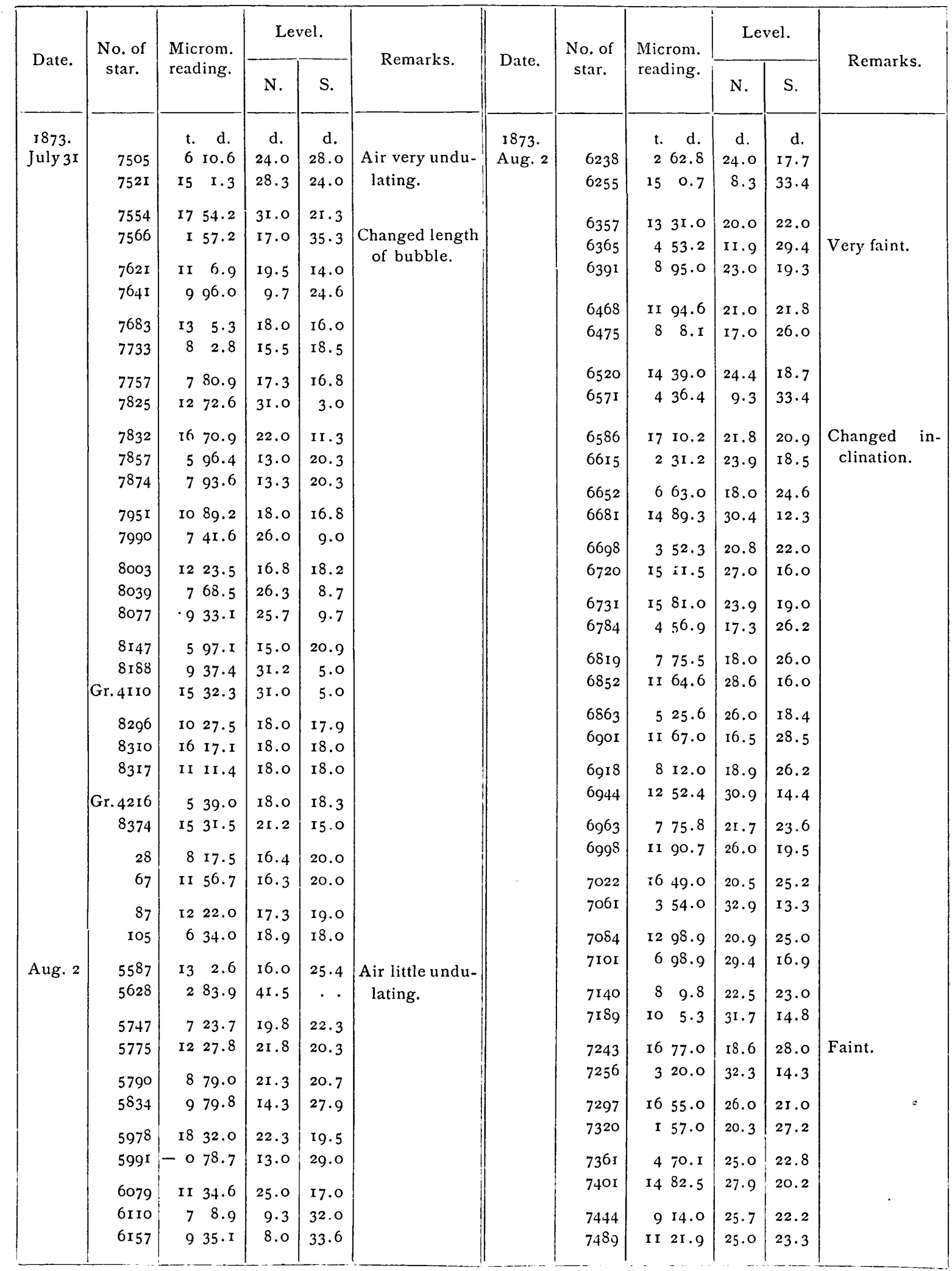


Observations for Latitude.-Station, Colorado Springs-Continued.

\begin{tabular}{|c|c|c|c|c|c|c|c|c|c|c|c|}
\hline \multirow{2}{*}{ Date. } & \multirow{2}{*}{$\begin{array}{l}\text { No. of } \\
\text { star. }\end{array}$} & \multirow{2}{*}{$\begin{array}{l}\text { Microm. } \\
\text { reading. }\end{array}$} & \multicolumn{2}{|c|}{ Level. } & \multirow{2}{*}{ Remarks. } & \multirow{2}{*}{ Date. } & \multirow{2}{*}{$\begin{array}{l}\text { No. of } \\
\text { star. }\end{array}$} & \multirow{2}{*}{$\begin{array}{l}\text { Nicrom. } \\
\text { reading. }\end{array}$} & \multicolumn{2}{|c|}{ Level. } & \multirow{2}{*}{ Remarks. } \\
\hline & & & N. & S. & & & & & N. & S. & \\
\hline \multirow[t]{36}{*}{$\begin{array}{l}1873 . \\
\text { Aug. } 2\end{array}$} & 7505 & $\begin{array}{l}\text { t. d. } \\
548.0\end{array}$ & $\begin{array}{c}\text { d. } \\
24.7\end{array}$ & $\begin{array}{r}\text { d. } \\
23.5\end{array}$ & & $\begin{array}{l}\text { I873. } \\
\text { Aug. } 4\end{array}$ & 357 & $\begin{array}{lr}\text { t. } & \text { d. } \\
6 & 96.9\end{array}$ & $\begin{array}{r}\mathrm{d} . \\
23.0\end{array}$ & $\begin{array}{r}\text { d. } \\
21.0\end{array}$ & \multirow{41}{*}{$\begin{array}{l}\text { Very heavy } \\
\text { wind. }\end{array}$} \\
\hline & $752 \mathrm{I}$ & 1445.6 & 24.6 & 23.5 & Cloudy. & & $44 \mathrm{I}$ & 1263.9 & 25.8 & 18.3 & \\
\hline & 7505 & 550.6 & 24.3 & 15.5 & Air good. & & 474 & 560.8 & 21.8 & 22.2 & \\
\hline & $752 \mathrm{I}$ & I4 23.7 & 3.0 & 37.0 & & & 514 & $16 \quad 5 \cdot 3$ & $\mathrm{I}_{4} .8$ & 29.0 & \\
\hline & 7554 & I7 79.8 & 22.6 & 17.3 & & 5 & 6238 & 296.0 & 19.8 & 15.0 & \\
\hline & 7566 & I 83.1 & $7 \cdot 4$ & 32.7 & & & 6255 & I5 $39 . \mathrm{I}$ & -1.0 & $35 \cdot 4$ & \\
\hline & 7621 & Io 19.3 & 23.9 & 16.2 & Changed the & & $665^{2}$ & $64 \mathrm{I} .2$ & 15.0 & 21.0 & \\
\hline & $764 \mathrm{I}$ & 910.0 & 5.0 & $35 \cdot 3$ & inclination. & & $668 \mathrm{I}$ & $\mathrm{I}_{4} 59.7$ & 21.6 & I 5.0 & \\
\hline & 7683 & I2 44.7 & 22.7 & I8.0 & & & 6698 & 341.8 & 20.2 & 16.0 & \\
\hline & 7733 & 726.9 & 23.0 & 18.0 & & & 6720 & I 487.3 & 16.0 & 21.0 & \\
\hline & 7757 & 721.0 & 25.2 & I5.8 & & & $673 \mathrm{I}$ & I5 13.4 & 20.2 & 17.0 & \\
\hline & 7825 & 124.6 & 20.7 & 21.0 & & & 6784 & $384 \cdot 3$ & 17.9 & 20.1 & \\
\hline & 7832 & I5 53.0 & 23.0 & I 8.3 & & & 6819 & 699.0 & 18.0 & 20.0 & \\
\hline & 7857 & 488.9 & 23.8 & 17.8 & & & 6852 & 10 95.0 & 28.0 & 10.2 & \\
\hline & 7874 & 686.5 & 23.6 & 18.0 & & & 6863 & 650.4 & 22.4 & 16.0 & \\
\hline & 7880 & 946.8 & 20.3 & 21.0 & Wrong setting. & & 6901 & I2 83.0 & 17.5 & 21.0 & \\
\hline & Gr. 3873 & I7 $49 \cdot 3$ & 30.0 & II. 3 & & & 6918 & $8 \quad 5.6$ & 17.3 & 21.2 & \\
\hline & $795 \mathrm{I}$ & I0 20.8 & 23.0 & 18.4 & & & $69+4$ & 1253.0 & 21.0 & 17.2 & \\
\hline & 7990 & 663.9 & 20.3 & 21.0 & & & 6963 & 827.0 & 20.8 & 18.0 & \\
\hline & 8003 & II 59.9 & 17.9 & 23.0 & & & 6998 & 1236.0 & 22.4 & 16.5 & \\
\hline & 8039 & 77.0 & 27.4 & $\mathrm{I}_{4} .0$ & & & 7022 & 1564.0 & 25.8 & I 3.9 & \\
\hline & 8077 & 868.5 & 27.8 & $\mathrm{I}_{4} .8$ & & & $706 \mathrm{I}$ & 264.9 & 19.0 & 20.0 & \\
\hline & 8147 & 542.0 & 19.8 & 22.8 & & & $70 S_{4}$ & 1222.5 & 21.3 & 17.3 & \\
\hline & 8188 & 891.0 & 33.7 & 9.0 & & & 7 IOI & 618.0 & 19.3 & 20.0 & \\
\hline & Gr.4110 & I4 83.0 & 32.7 & 9.8 & & & & 7798 & & ? & \\
\hline & 8296 & 951.0 & 28.0 & $I_{4} .5$ & & & 7540 & $\begin{array}{l}771.8 \\
055.2\end{array}$ & $\mid \begin{array}{l}20.0 \\
8.0\end{array}$ & I9.2 & \\
\hline & 8310 & I 546.0 & $x_{4} .0$ & 28.6 & & & 7189 & 955.2 & 18.0 & $21 \cdot 3$ & \\
\hline & 8317 & Io 38.9 & 13.5 & 29.2 & & & 7243 & I6 70.0 & 20.3 & 19.0 & \\
\hline & Gr. 4216 & $5 \quad 4 \cdot 3$ & 24.7 & 18.0 & . & & 7256 & 319.6 & $x 6.6$ & 23.0 & \\
\hline & $8_{374}$ & It 02.5 & 16.6 & 26.0 & & & 7297 & 1644.0 & 23.9 & 15.8 & \\
\hline & 28 & 799.1 & 25.0 & 18.0 & & & 7320 & I 60.5 & 9.0 & 30.8 & \\
\hline & 67 & II 26.2 & 20.3 & 22.3 & & & $736 \mathrm{r}$ & 418.0 & 23.3 & 16.0 & \\
\hline & 87 & II 44.4 & 22.0 & 20.8 & & & $740 z$ & 1412.7 & 9.8 & 30.0 & \\
\hline & 105 & 570.3 & 25.6 & 17.3 & & & $7+44$ & Io 34.0 & 18.0 & 21.8 & \\
\hline & $\mathrm{I} 2 \mathrm{I}$ & I 58.0 & 17.3 & 25.7 & & & 7489 & 1240.9 & $24 \cdot 3$ & 15.7 & \\
\hline & I 78 & 588.5 & 28.8 & 14.5 & & & 7505 & 571.0 & 24.5 & 15.0 & \\
\hline \multirow[t]{5}{*}{ - } & $20 \mathrm{r}$ & $4 \div 9.3$ & 19.0 & 24.3 & & & $752 \mathrm{I}$ & 1460.3 & I0.8 & 29.0 & \\
\hline & 1 & 935.0 & 55.3 & 23.0 & & & $755-7$ & $: 830.9$ & 23.5 & $=5.6$ & \\
\hline & 250 & I $5 \div 2.0$ & 27.0 & 16.3 & & & 7565 & $22 \pi .0$ & $I \div .3$ & 25.5 & \\
\hline & $2 \hat{5} 5$ & $9=0.5$ & :s. & 25.2 & & & $762 I$ & 977.0 & 20.0 & 21.0 & \\
\hline & 330 & II $\mathrm{II} .2$ & 32.0 & 12.0 & & & $76+1$ & $8+4.8$ & 23.3 & 13.0 & \\
\hline
\end{tabular}


Observations for Latitude.-Station, Colorado Springs-Continued.

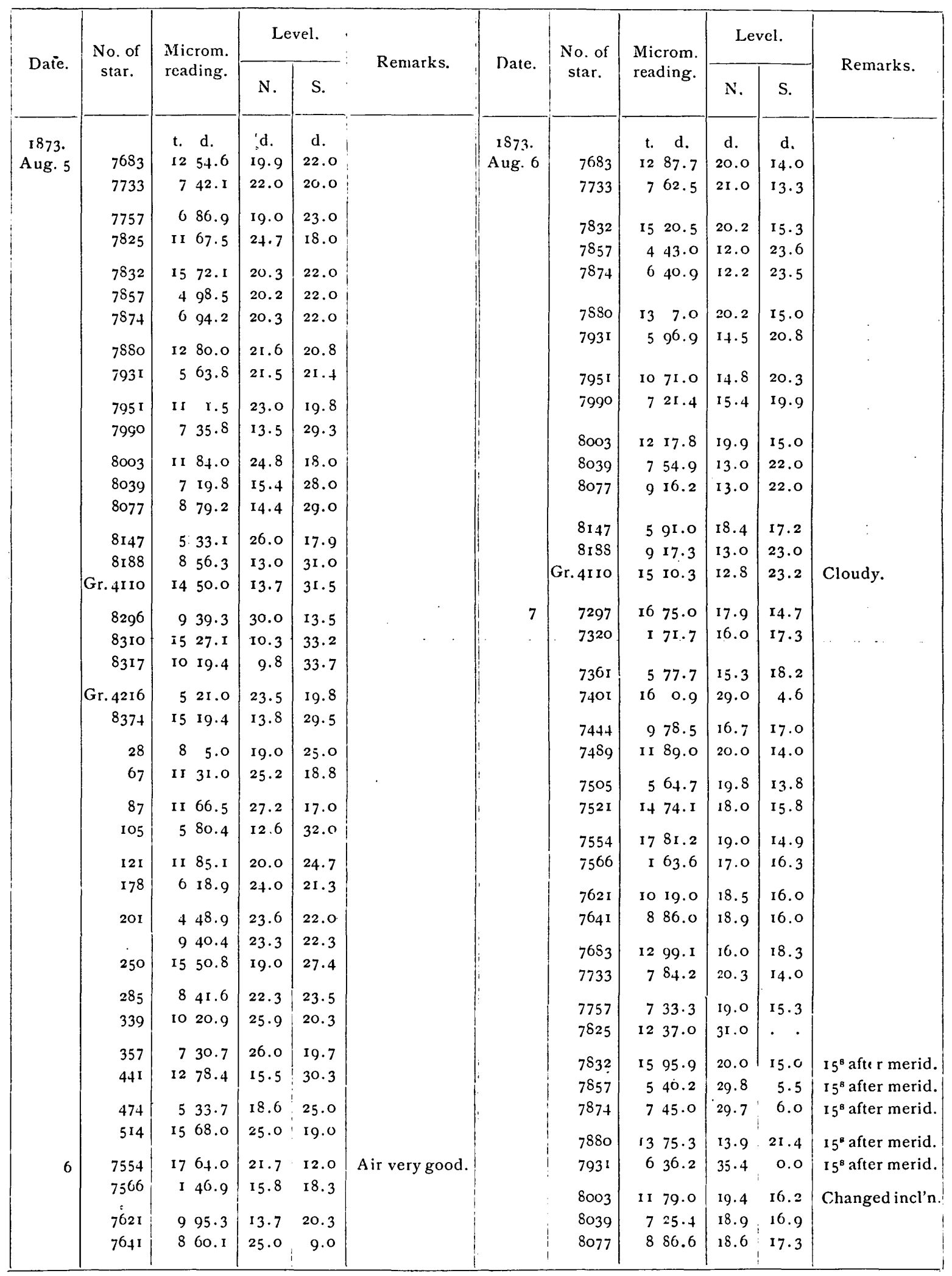


74

Observations for Latitude._Station, Colorado Springs-Continued.

\begin{tabular}{|c|c|c|c|c|c|c|c|c|c|c|c|}
\hline \multirow{2}{*}{ Date. } & \multirow{2}{*}{$\begin{array}{l}\text { No. of } \\
\text { star. }\end{array}$} & \multirow{2}{*}{$\begin{array}{l}\text { Microm. } \\
\text { reading. }\end{array}$} & \multicolumn{2}{|c|}{ Level. } & \multirow{2}{*}{ Remarks. } & \multirow{2}{*}{ Date. } & \multirow{2}{*}{$\begin{array}{l}\text { No. of } \\
\text { star. }\end{array}$} & \multirow{2}{*}{$\begin{array}{l}\text { Microm. } \\
\text { reading. }\end{array}$} & \multicolumn{2}{|c|}{ Level. } & \multirow{2}{*}{ Remarks. } \\
\hline & & & N. & S. & & & & & N. & S. & \\
\hline \multirow{22}{*}{$\begin{array}{l}\text { I873. } \\
\text { Aug. } 7\end{array}$} & & t. d. & d. & d. & & & & & & & \multirow{19}{*}{$\begin{array}{l}\text { Cloudy, heavy } \\
\text { rain,and storm. }\end{array}$} \\
\hline & 8147 & 580.5 & 19.8 & 16.8 & & $\begin{array}{l}1873 . \\
\text { Aug. }\end{array}$ & 5747 & 725.0 & $\begin{array}{l}\text { a. } \\
\text { I } 8.0\end{array}$ & $\begin{array}{c}\text { a. } \\
2 \mathrm{~J} .6\end{array}$ & \\
\hline & 8188 & 921.2 & 20.3 & 16.6 & & & 5775 & 1236.0 & 27.0 & 13.0 & \\
\hline & Gr. 4 IIIO & I 514.2 & 20.3 & 16.6 & & & & & & & \\
\hline & & & & & & & 5790 & 922.8 & 19.5 & 20.4 & \\
\hline & 8290 & IO $45 \cdot 3$ & 22.0 & 14.8 & & & 5834 & $10 \mathrm{II} .7$ & 22.0 & 18.0 & \\
\hline & 8310 & I6 46.5 & 17.3 & I9. 8 & & & & & & & \\
\hline & 8317 & I I 38.9 & 17.0 & 20.2 & & & $587 \mathrm{I}$ & 846.8 & 22.3 & 17.9 & \\
\hline & Gr. 4216 & 485.3 & ro.6 & 17.6 & & & 5927 & IO 34.2 & 16.0 & 25.0 & \\
\hline & 8374 & 1490.6 & 8.3 & 29.0 & & & 5978 & 1874.0 & 18.5 & 22.6 & \\
\hline & 28 & 842.0 & 22.0 & 15.2 & & & $599 \mathrm{I}$ & -062.7 & 30.3 & 10.8 & \\
\hline & 67 & II 76.1 & 9.7 & 28.2 & & & 6079 & 1224.2 & 24.0 & 17.9 & \\
\hline & & & & & & & 6110 & 790.9 & 16.0 & 26.0 & \\
\hline & 87 & II 92.6 & 18.0 & I9.6 & & & 6157 & 10 16.0 & 16.3 & 25.9 & \\
\hline & 105 & $\begin{array}{ll}6 & 8.0\end{array}$ & 14.7 & 22.9 & & & & & & & \\
\hline & I 21 & I 88.3 & 17.3 & 20.2 & & & 7444 & $10 \quad 1.0$ & 19.0 & $24 \cdot 3$ & \\
\hline & I 78 & 630.3 & 15.2 & 22.2 & & & 7489 & 1223.2 & 34.0 & $9 \cdot 7$ & \\
\hline & \multirow{2}{*}{201} & & & & & & 7505 & $6 \mathrm{I}_{4} .6$ & 26.0 & 16.0 & \\
\hline & & $\begin{array}{l}459.9 \\
954.6\end{array}$ & $\begin{array}{l}20.0 \\
21.8\end{array}$ & $\begin{array}{l}17.3 \\
15.9\end{array}$ & & & $752 \mathrm{I}$ & 1528.7 & 21.8 & $2 \mathrm{I} .8$ & \\
\hline & 250 & 1577.0 & 1.0 & 36.0 & & & 8003 & II 93.5 & 25.0 & 19.5 & \multirow{3}{*}{$\begin{array}{l}\text { Cloudy ; thro' } \\
\text { clouds. } \\
\text { Setting of mic. } \\
\text { uncertain. }\end{array}$} \\
\hline & 474 & 568.4 & 17.3 & 20.2 & & & 8039 & 752.0 & 30.0 & I 4.5 & \\
\hline & $5 I_{4}$ & I6 24.0 & $5 \cdot 9$ & 31.8 & Cloudy. & & 8077 & 923.2 & 30.4 & $\mathrm{I} 4.0$ & \\
\hline
\end{tabular}


Computations for Latitude.-Station, Colorado Springs.

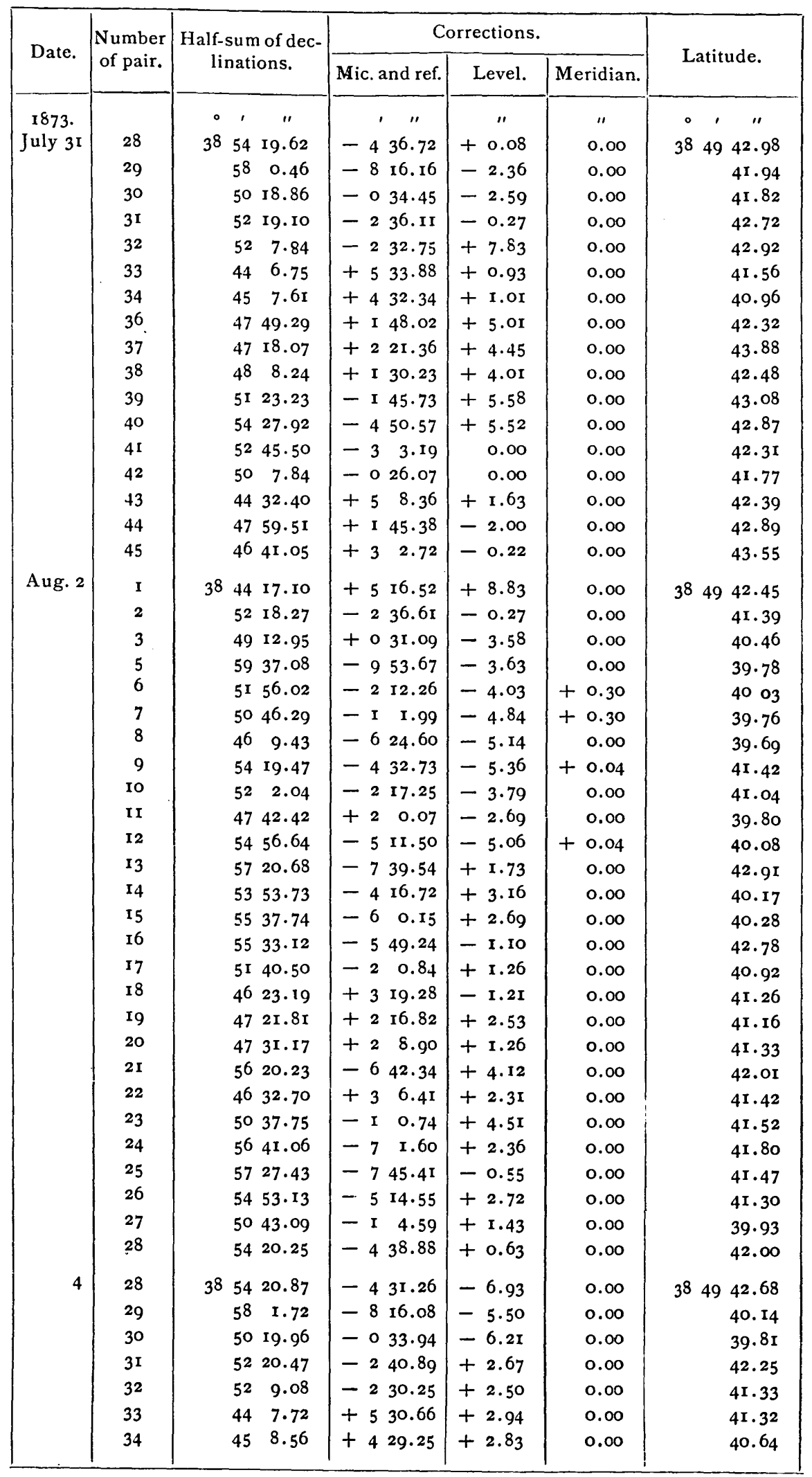


Computation for Latitude.-Station, Colorado Springs-Continued.

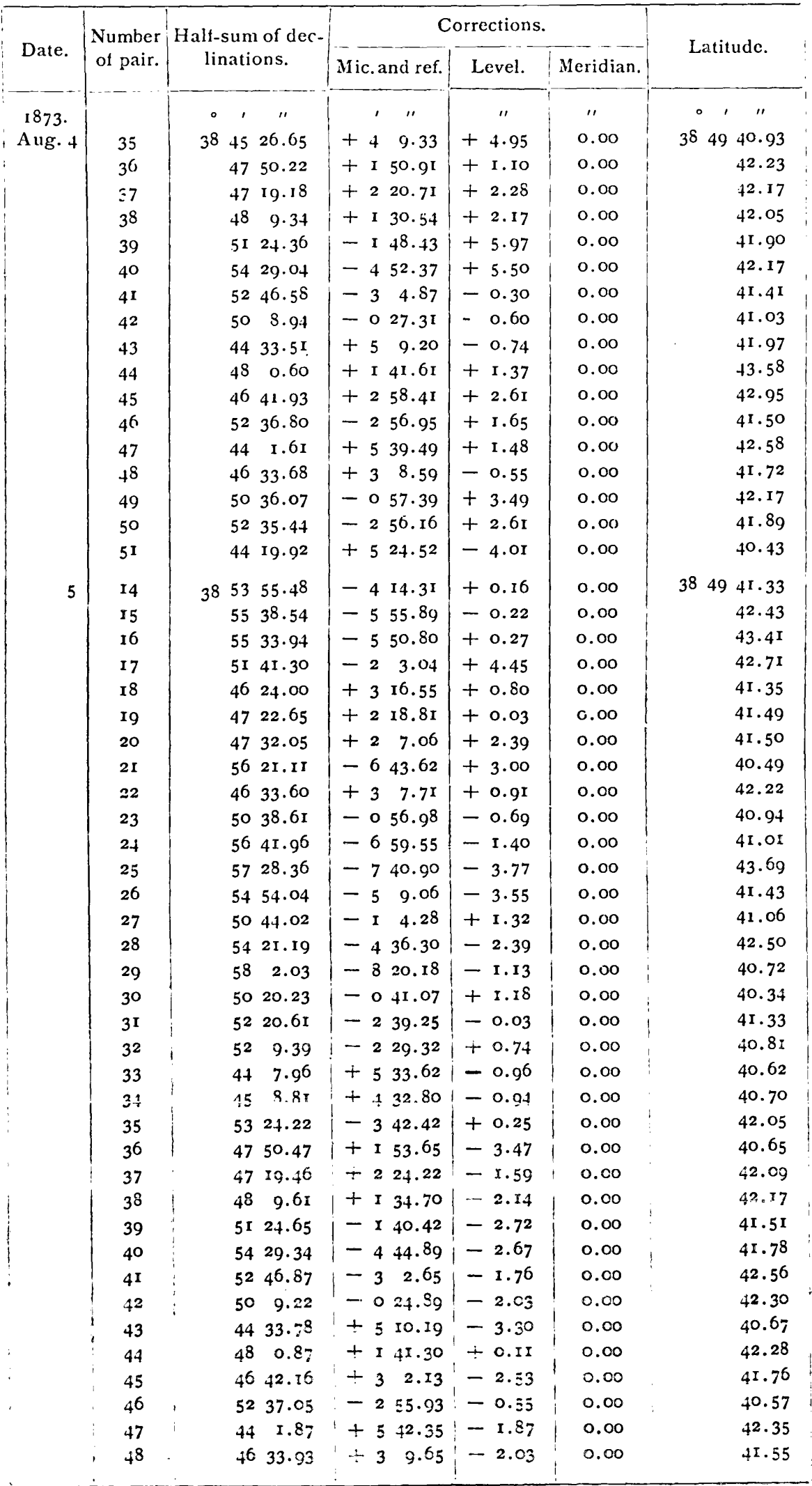


Computations for Latitude.-Station, Colorado Springs-Continued

\begin{tabular}{|c|c|c|c|c|c|c|}
\hline \multirow{2}{*}{ Date. } & \multirow{2}{*}{$\begin{array}{l}\text { Number } \\
\text { of pair. }\end{array}$} & \multirow{2}{*}{$\begin{array}{l}\text { Half-sum of dec- } \\
\text { linations. }\end{array}$} & \multicolumn{3}{|c|}{ Corrections. } & \multirow{2}{*}{ Latitude. } \\
\hline & & & Mic. and ref. & Level. & Meridian. & \\
\hline \multirow{15}{*}{$\begin{array}{l}\text { I } 873 . \\
\text { Aug. } 5\end{array}$} & & $0,1$. & , & " & " & $\circ, 11$ \\
\hline & 49 & $3^{8} \quad 50 \quad 3^{6} \cdot 3^{6}$ & $-\circ 55.7 \mathrm{I}$ & $+\mathrm{I} .2 \mathrm{I}$ & 0.00 & $\begin{array}{lll}38 & 49 & 41.86\end{array}$ \\
\hline & 50 & $5^{2} 35.67$ & -250.17 & -2.34 & 0.00 & 43.16 \\
\hline & $5 \mathrm{I}$ & 4420.15 & +521.35 & -0.11 & 0.00 & $41 \cdot 39$ \\
\hline & 29 & $3^{8} \quad 5^{8} \quad 2.34$ & $-822.4 \mathrm{I}$ & +1.98 & 0.00 & $3849 \quad 4 \mathrm{r} \cdot 9 \mathrm{r}$ \\
\hline & 30 & $5020.5 \mathrm{I}$ & -042.00 & +2.58 & 0.00 & 41.09 \\
\hline & $3 r$ & 5220.90 & -243.18 & +3.77 & 0.00 & 41.49 \\
\hline & 33 & $44 \quad 8.20$ & +534.84 & -1.84 & 0.00 & 41.20 \\
\hline & 34 & $45 \quad 9.05$ & +433.33 & -1.76 & 0.00 & 40.62 \\
\hline & 35 & $5324 \cdot 54$ & -340.63 & -0.30 & 0.00 & $43.6 \mathrm{I}$ \\
\hline & 36 & 4750.71 & + I 54.85 & -2.75 & 0.00 & $42.8 \mathrm{I}$ \\
\hline & 37 & $47 \quad 19.75$ & +223.82 & -1.13 & 0.00 & $42.44^{\circ}$ \\
\hline & 38 & $4^{8} \quad 9.89$ & +133.71 & -1.13 & 0.00 & 42.47 \\
\hline & 39 & 5124.94. & - I 41.39 & -2.42 & 0.00 & 41.13 \\
\hline & 40 & 5429.62 & -445.64 & -2.53 & 0.00 & 41.45 \\
\hline \multirow[t]{24}{*}{7} & 25 & $\begin{array}{lll}38 & 57 & 28.97\end{array}$ & -747.05 & -0.52 & 0.00 & $\begin{array}{lll}38 & 49 & 41.40\end{array}$ \\
\hline & 26 & 5454.62 & -517.90 & +3.16 & 0.00 & 39.88 \\
\hline & 27 & $5044 \cdot 96$ & - I $5.4 \mathrm{I}$ & +1.57 & 0.00 & $4 \mathrm{I} \cdot \mathrm{I} 2$ \\
\hline & 28 & 5421.81 & -44215 & +2.23 & 0.00 & 41.89 \\
\hline & 29 & $5^{8} \quad 2.66$ & -822.63 & +1.32 & 0.00 & $4 \mathrm{r} \cdot 35$ \\
\hline & 30 & $50 \quad 20.77$ & $-04 \mathrm{i} \cdot 32$ & +1.48 & 0.00 & 40.93 \\
\hline & $3 \mathrm{I}$ & 5221.20 & - 239.99 & +1.10 & 0.00 & $42.3 \mathrm{I}$ \\
\hline & 32 & 52 1о.ог & -246.49 & +9.54 & 0.00 & 43.06 \\
\hline & 33 & 448.46 & +526.19 & +8.06 & 0.00 & 42.71 \\
\hline & 34 & $45 \quad 9.29$ & $+429.4 \mathrm{I}$ & +7.89 & 0.00 & 41.59 \\
\hline & 35 & 5324.86 & -349.63 & +7.70 & +0.06 & 42.99 \\
\hline & 37 & $47 \quad 20.02$ & +230.93 & $+\mathrm{I} .43$ & 0.00 & $42 \cdot 38$ \\
\hline & 38 & 48 10.18 & + I 30.85 & +1.24 & 0.00 & 42.27 \\
\hline & 39 & 5125.22 & - I. 45.86 & +1.84 & 0.00 & $4 \mathrm{I} .20$ \\
\hline & 40 & 5429.9 I & $-450.1 \mathrm{I}$ & +1.84 & 0.00 & 41.64 \\
\hline & $4 \mathrm{I}$ & 5247.44 & -36.79 & +1.29 & 0.00 & $4^{1} .94$ \\
\hline & 42 & $50 \quad 9.79$ & - 029.08 & +1.10 & 0.00 & $4 \mathrm{I} .8 \mathrm{I}$ \\
\hline & 43 & 4434.35 & +512.31 & -5.14 & 0.00 & 41.52 \\
\hline & 44 & $48 \quad$ I. 43 & + I 43.79 & -3.22 & 0.00 & 42.00 \\
\hline & 45 & $46 \quad 42.62$ & +31.67 & -2.69 & 0.00 & +1.60 \\
\hline & 46 & 5237.52 & -253.38 & -2.72 & 0.00 & $41 \cdot 42$ \\
\hline & 47 & $\begin{array}{ll}44 & 2.3^{8}\end{array}$ & +547.07 & -8.88 & 0.00 & 40.57 \\
\hline & 48 & $46 \quad 34.45$ & +313.37 & -8.00 & 0.00 & 39.82 \\
\hline & $5 \mathrm{I}$ & 4420.60 & +527.95 & -7.92 & 0.00 & 40.63 \\
\hline \multirow{10}{*}{9} & 2 & $\begin{array}{lll}38 & 52 & 19.24\end{array}$ & -238.76 & +2.86 & 0.00 & $3^{8} 49 \quad 43.34$ \\
\hline & 3 & $49 \times 3.96$ & +027.62 & +0.85 & 0.00 & 42.43 \\
\hline & 4 & $48 \quad 44.25$ & +058.23 & -1.26 & 0.00 & 41.22 \\
\hline & 5 & $593^{8.12}$ & -Io 1.74 & +4.23 & 0.00 & $40.6 \mathrm{I}$ \\
\hline & 6 & $51 \quad 57.25$ & -214.62 & -1.07 & 0.00 & $41 \cdot 56$ \\
\hline & 7 & $5047.5 \mathrm{I}$ & - I 4.69 & -0.96 & 0.00 & $4 \mathrm{I} .86$ \\
\hline & 27 & $5045.2 \mathrm{I}$ & -19.03 & +5.23 & 0.00 & $4 \mathrm{I} \cdot 4 \mathrm{I}$ \\
\hline & 28 & $54 \quad 22.43$ & -444.00 & +2.86 & $0 . \infty 0$ & $4^{1.29}$ \\
\hline & 37 & $47 \quad 20.56$ & +217.17 & +5.77 & +0.19 & 43.88 \\
\hline & 38 & $48 \quad 10.77$ & +226.10 & +6.02 & +0.19 & $43 \cdot 28$ \\
\hline
\end{tabular}


The observations for latitude were made under very unfavorable circumstances. Undulations in the atmosphere, heavy winds, and great changes in temperature constantly affected the instrument.

It is customary in this office to select for latitude only thirty-five pairs of stars, which have to be observed on five different nights. But the observer is dependent upon the weather, and is frequently disappointed, although after waiting I preferred to select a greater number of pairs, believing that the final result of a latitude is better when depending upon various star-places, giving the probability that the errors resulting from the declination-places of the stars will more nearly compensate each other, and that this part of the probable error of the final result will come within that resulting from observation alone.

The mean latitude is obtained by taking the mean of all single results. For the different days the mean latitude is found to be as follows:

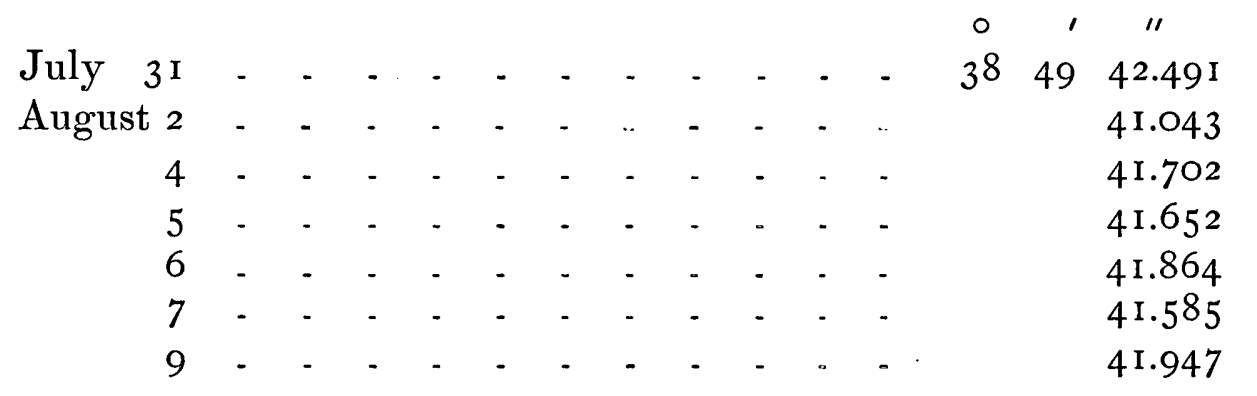

showing a great difference between the first and second day, arising from the disturbed condition of the air.

The probable error of one observation is,

$$
\varepsilon_{0}= \pm 0.6745 \wedge \sqrt{\overline{\frac{\Sigma(v)^{2}}{n-1}}}
$$

where $v$ is the difference between the mean results and the single results and $n$ the number of observations; therefore the probable error of the mean result is,

$$
\varepsilon_{r}= \pm 0.6745 \wedge \sqrt{\frac{\sum(v)^{2}}{n(n-I)}}
$$

If it is proper to place all the observations in the final result with the same weight (as in determining the longitude of a station from different nights' work) the formula should be used in this way; but in determining the latitude of a station, every single result obtained also depends upon the places of the stars forming the different pairs. It is certainly wrong to determine the probabie error of the latitude-resiat by this formula, (it would give, for latitude of Colorado Springs, a probable error less than $o^{\prime \prime}$. or, ) 
though it is frequently done. If nearly the same number of stars are observed every night under the same conditions, I should prefer to determine the probable error of the final result after the manner of $\mathrm{Mr}$. John H. Clark.

Let-

Probable error of one pair of stars, including constant errors of zenith-telescope observations - _ _ _ _ _ - $=\varepsilon_{p}$

Probable error of one observation - - - . - . - - - $=\varepsilon_{\mathrm{o}}$

Number of pairs used at the station - - - - - - - $=m$

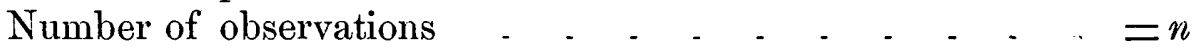

then probable error of the final result,

$$
\varepsilon_{r}= \pm \sqrt{\frac{\varepsilon_{p}^{2}}{m}+\frac{\varepsilon_{o}^{2}}{n}}
$$

The formula shows that if the stars used are not very good, it is then better to select a larger number of pairs of stars, giving the probability that the final result will be more independent of the declinations.

From all the observations of pairs of stars observed on three or more nights, I find the probable error of one observation-

$$
\varepsilon_{\mathrm{o}}= \pm \mathrm{O}^{\prime \prime} .428
$$

and that of the final result,

$$
\varepsilon_{r}= \pm 0^{\prime \prime} .035
$$

Taking the value for $\varepsilon_{p}$, found by Maj. C. B. Comstock, of the United States Lake Survey, for stars taken from Professor Safford's Catalogue for 981 Stars, $\pm 0^{\prime \prime} .53$, the probable uncertainty of the final result will be, $\pm \mathrm{o}^{\prime \prime} .082$.

Resulting Astronomical Co-ordinates for the Astronomical Monument at Colorado Springs, Colorado Territory, using, for the longitude of Washington and Salt Lake, the same data as in Clark's report.

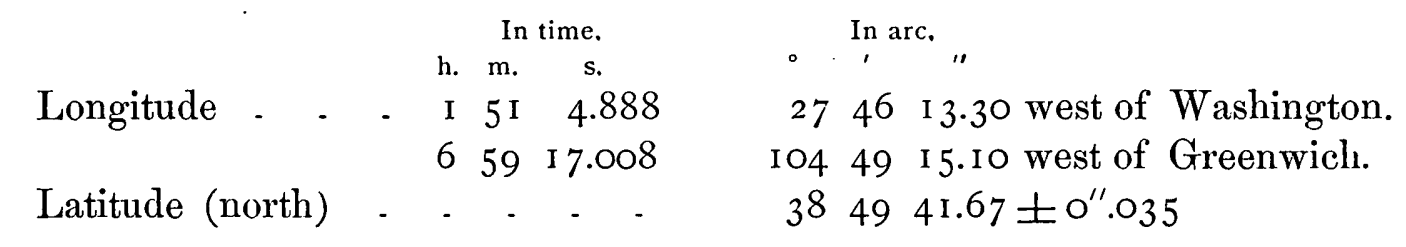

Respectfully submitted.

First Lieut. Geo. M. Wheeler,

DR. F. KAMPF, Civilian Astronomical Assistant.

Corps of Engineers, in charge. 


\section{United States Engineer Office, Geographircal and Geological Explorations and Surveys}

West of the iоOth Meridian, May i, i 873.

Memorandum of instructions for conducting obscrations for longitude and latitude at a main or primary astronomical station for the field-stason of 1873 .

The fixed observatory, with which connection will be made, is in Temple Square, Salt Lake City, Utah, and in charge of Assistant John H. Clark. Every observer is responsible for the receiving of the signals from the connecting-stations for certain reductions of results, as full as possible, and will follow such other instructions that may, from time to time, be given. This station will be changed during the season, probably in the month of August, to the United States engineer observatory, to be constructed at Ogden, Utah.

\section{I.-Observations for Longitude.}

In carrying on the astronomical campaign, preference will be given to the longitude-results for those nights that are clear at both stations; that is, the observers in charge, after having completed the necessary arrangements for the observations and exchange of signals, will, from this time on, exchange signals each night that shall be fair at the two stations, unless unavoidable difficulties arise to prevent.

It will be carefully observed that weather-signals are sent by telegraph each evening about $6 \mathrm{p}$. m. while the exchanges are going on and until the full series at a station shall have been completed. In case of uncertainty as to the possibility for observing for any one night, this may be indicated in the first dispatch; and subsequent dispatch or dispatches, between this time and Io p. m., shall determine whether exchanges shall be made that night or not.

Some convenient and concise form will be adopted for the weather-signals, and in no case must there be a failure as regards forwarding them.

It may be admissible, contingent upon the stage of the prosecution of the work, should the weather at Salt Lake at about $6 \mathrm{p}$. m. look unfavorable, that the observer at the distant station should at once conclude to take that night for latitude-work, which may be done after informing the observer at the Salt Lake or the receiving station.

For the conducting of the observations for the exchanges for a single night, the following instructions will be adhered to: The transit of stars for time-leterminations, consisting of not less than three time and two circumpolar stars in each position of the instrument, both before and after the transmission of the signals, will be taken. Of course, should the night be sufficiently clear, so that it seems possible to make the time-determinations at both stations, by the modification of the above, so that at one or both of the stations the transits of stars may be made entirely before or entirely after the sending of the signals, the strict following-out of the above is not necessary.

These instructions are made with the understanding that a twenty-six or thirty- 
two inch Würdemann instrument is used, with recording-apparatus in shape of a chironograph or register.

The time set for the transmission of signals should be as near 9.30 p. m. local time of the Salt Lake meridian as possible; and great care should be taken that both observers shall be on hand simultaneously, so that as little delay as possible shall ensue in the use of the telegraph-wires.

In the transmission of signals the record is to be made upon the chronograph or register at the two places over a space of five minutes in time, the connecting or Salt Lake station sending for the first five minutes and the distant station receiving, and vice revse.

In addition to these, which may be known as the chronograph-signals, arbitrary signals will be sent, by the use of a break-circuit key, at about ten seconds apart, at fractional parts of a second, making a series of thirty-one arbitrary signals during the five minutes.

In order that the observer at a station may conclude that he has accomplished six nights of first-class observations, it becomes necessary that there should be an exchange of the approximate results. Each observer will therefore send to the other, upon each subsequent night or as soon thereafter as practicable, the approximate error of his chronometer and the mean of seven arbitrary signals, sent and received, selected from the middle of the set of thirty-one. This may be concisely expressed in a telegram.

For the fuil satisfaction of an observer at the distant station, such further computation shall be sent and received as shall seem necessary to a clear understanding of the case; as an observer will be held responsible should he leave the station and go to another before he is certain that the results upon final computation would prove satisfactory.

\section{2.-Observations for Latitude.}

These will be conducted through five complete and clear nights, so that there shall not be less than 175 pairs of observations upon 35 separate and distinct pairs of stars, each pair of which observations shall give a first-class result.

These instructions are furnished to the observers with a view to their clearly understanding the class of results intended, and will always be carried out, unless unforeseen difficulties arise, in which event, as the observers will not hereafter be within speedy communication of these headquarters, it will be necessary for them to adopt immaterial modifications upon their own responsibility. It is, of course, understood that each observer is responsible for and receives the credit of his own work.

A report will be made by each observer, at intervals not exceeding fifteen days, of the work under his charge.

A full daily journal will be kept by each observer. Great care will be taken as to the character of the record upon this journal, which should be clear and explicit. The position of the station in reference to surrounding natural objects should be clearly described, and imperishable meridian-marks firmly planted. When possible, a special survey and plat will be made. 
The day following an exchange of the chronograplic record of time and exchange. signals, said signais wili be curefully cojied into a record-book, which, like all of her records, must be made in drulicate.

It is intended that computations in the field shall be carried on to such an extent as circumstances may permit.

The time that can be ailotted to each of these stations, in order to accomplish the expected results for the season, is from twenty to twenty-five nights; and as observations are required only ror eleven nights, the remaining interval should be employed in computation.

The order of sequence for an astronomical report is furnished herewith, and the records and journal will be so kept as to comprise all the data necessary for the fuil expression of results in accordance therewith.

\section{Official :}

GEO. M. WHEELER,

First Lieutenant Corps of Engineers, in charge. 